\title{
Learning in urology : designing simulator based skills : training \& assessment
}

Citation for published version (APA):

Tjiam, I. M-I. (2014). Learning in urology : designing simulator based skills : training \& assessment.

[Doctoral Thesis, Maastricht University]. Maastricht University. https://doi.org/10.26481/dis.20140917it

Document status and date:

Published: 01/01/2014

DOI:

10.26481/dis.20140917it

Document Version:

Publisher's PDF, also known as Version of record

\section{Please check the document version of this publication:}

- A submitted manuscript is the version of the article upon submission and before peer-review. There can be important differences between the submitted version and the official published version of record.

People interested in the research are advised to contact the author for the final version of the publication, or visit the DOI to the publisher's website.

- The final author version and the galley proof are versions of the publication after peer review.

- The final published version features the final layout of the paper including the volume, issue and page numbers.

Link to publication

\footnotetext{
General rights rights.

- You may freely distribute the URL identifying the publication in the public portal. please follow below link for the End User Agreement:

www.umlib.nl/taverne-license

Take down policy

If you believe that this document breaches copyright please contact us at:

repository@maastrichtuniversity.nl

providing details and we will investigate your claim.
}

Copyright and moral rights for the publications made accessible in the public portal are retained by the authors and/or other copyright owners and it is a condition of accessing publications that users recognise and abide by the legal requirements associated with these

- Users may download and print one copy of any publication from the public portal for the purpose of private study or research.

- You may not further distribute the material or use it for any profit-making activity or commercial gain

If the publication is distributed under the terms of Article $25 \mathrm{fa}$ of the Dutch Copyright Act, indicated by the "Taverne" license above, 


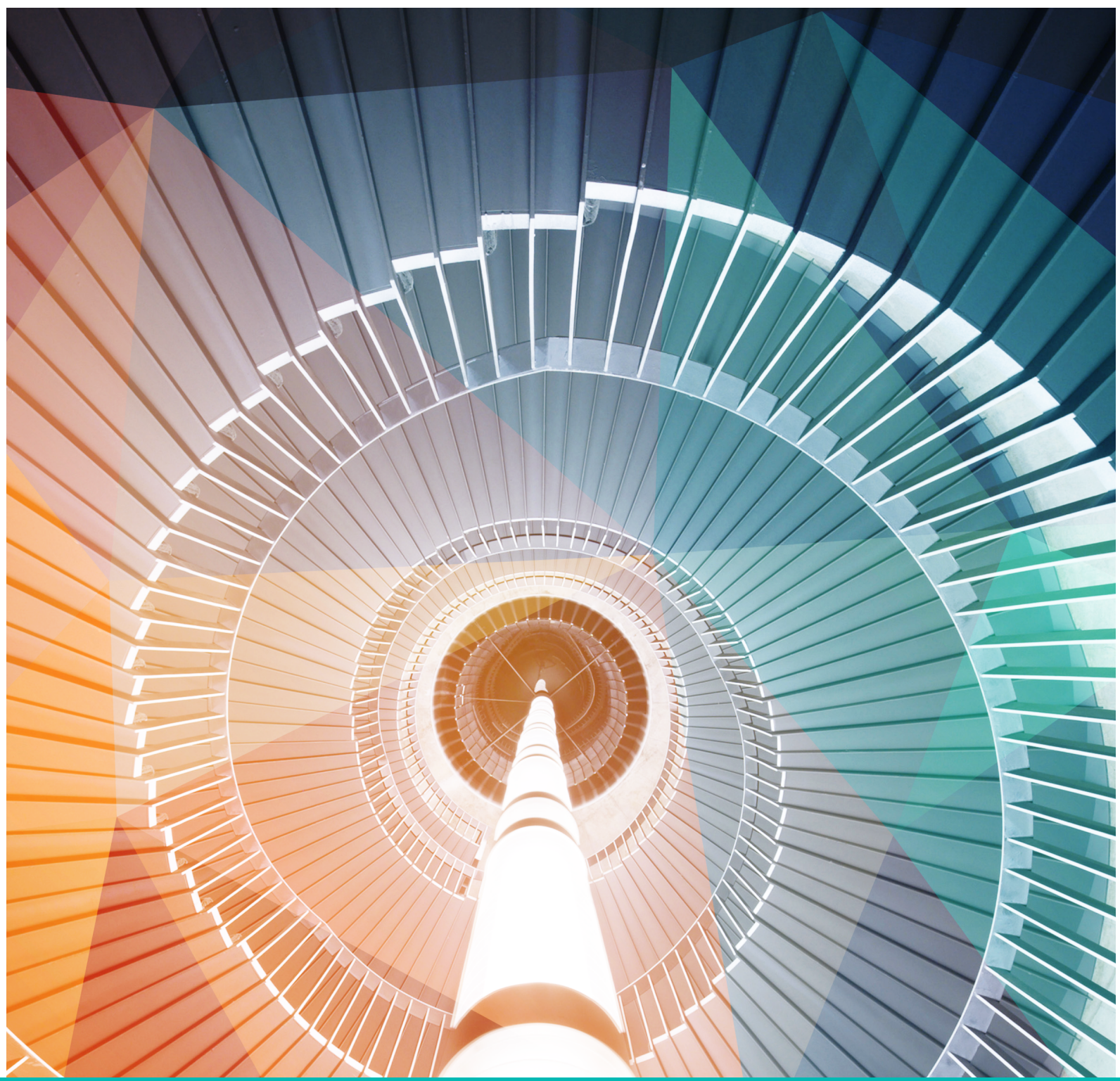

Learning in Urology

Designing Simulator Based Skills

Training \& Assessment
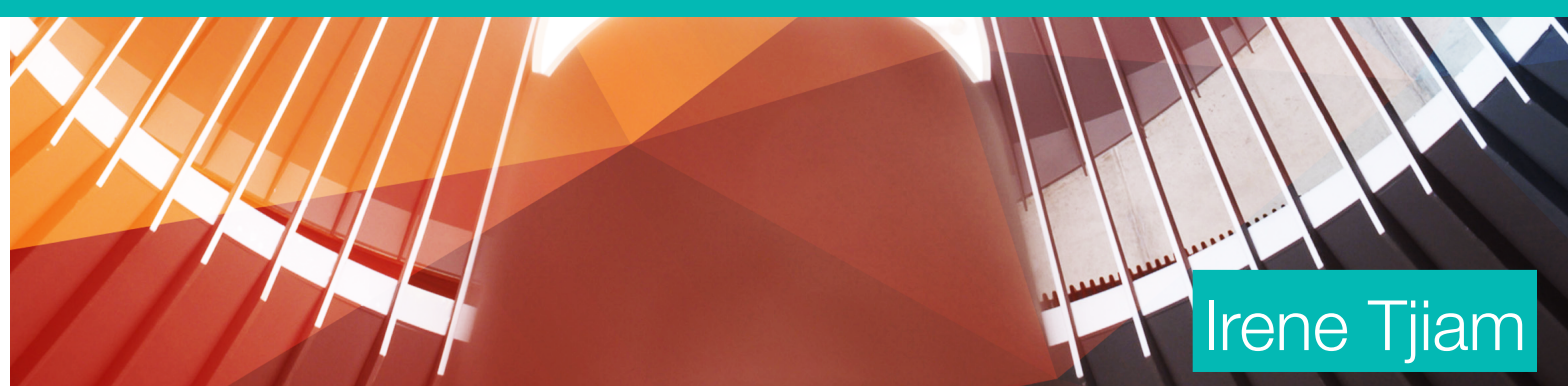

Learning in Urology

\section{Designing Simulator Based Skills Training \& Assessment}


Download this thesis via:

http://www.e-pubs.nl?epub=i.tjiam

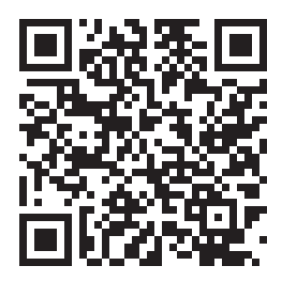

The Research reported here was carried out at

\section{DMastricht University in Learning!}

In the School of Health Profession Education

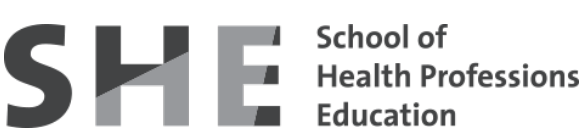

In collaboration with:

the department of urology, Catharina Hospital, Eindhoven, the Netherlands

the department of urology, Radboud UMC, Nijmegen, the Netherlands

This publication of this thesis was financially supported by:

Astellas, AmericanMedicalSystems , Applied Medical, BayerHealthcare, Biomedic

Nederland BV, Coloplast, Epona Medical, Erbe, Dutch Society for Simulation in

Healthcare, GlaxoSmithKlein, Hoogland Medical, Ipsen Farmaceutica BV, Mundipharma,

Olympus Nederland BV, Pfizer, ZambonGroup

ISBN: 978-94-6169-530-7

Lay out \& Printing: Optima Grafische Communicatie BV, Rotterdam, the Netherlands Cover design: Frederic Lie

Copyright $\odot 2014$ by Irene Tjiam, Eindhoven, The Netherlands

All rights are reserved. No part of this thesis may be reproduced, stored or transmitted in any form or by any means, without prior permission of the author, or when applicable, of the publishers of the scientific paper. 


\title{
Learning in Urology Designing simulator based skills Training \& Assessment
}

\author{
PROEFSCHRIFT \\ ter verkrijging van de graad van Doctor \\ aan de Universiteit Maastricht, \\ op gezag van de Rector Magnificus, Prof. dr. L.L.G. Soete, \\ volgens het besluit van het College van Decanen,
}

in het openbaar te verdedigen op woensdag 17 september 2014 om 16.00 uur

door

Irene Mei-Ing Tjiam 


\section{Promotores:}

Prof. dr. A.J.J.A. Scherpbier

Prof. dr. J.A. Witjes, Radboud UMC

\section{Copromotores:}

Dr. A.J.M. Hendrikx, Catharina Ziekenhuis Eindhoven

Dr. B.M.A. Schout, Medisch Centrum Alkmaar

\section{Manuscript Commissie}

Prof. dr. L.P.S. Stassen (voorzitter)

Prof. dr. I. Heyligers

Prof. dr. R.C.M. Pelger, LUMC

Prof. dr. F.W.J.M. Smeenk

Dr. P.W. Teunissen 


\section{TABLE OF CONTENTS}

1 Introduction 7

$2 \quad$ Program for Laparoscopic Urological Skills: A newly developed 21 and validated educational program

Urology 2012; 79(4): 815-20

3 Program for laparoscopic urological skills assessment: Setting certification standards for residents

Minimally Invasive Therapy 2013; 22(1): 26-32

4 Results of the European Basic Laparoscopic Urological Skills

Examination

European Urology 2014; 65(2): 490-6

5 Evaluation of the educational value of a virtual reality TURP simulator according to a curriculum based approach

Simulation in Healthcare 2014; accepted

6 Designing simulator based training: An approach integrating cognitive task analysis and four-component instructional design

Medical Teacher 2012; 34(10): e698-e707

7 Ergonomics in Endourology and Laparoscopy: An overview of musculoskeletal problems in urology Journal of Endourology 2014; 28(5): 605-11

8 General Discussion and Future Perspectives

Summary

Samenvatting

Curriculum Vitae

List of Publication

Dankwoord 





\section{INTRODUCTION}

Endourological procedures are well known in urological practice, wherein operating instruments enter the patients' body through a natural orifice, the urethra. Cystoscopy (inspection of the bladder surface), transurethral resection of the prostate (TURP), transurethral resection of bladder tumour (TURBT) and ureterorenoscopy (inspection of the higher urinary tract) are the main transurethral procedures. ${ }^{1}$ In the beginning of the $19^{\text {th }}$ century the first endoscope including a light transporter and a working channel was developed. Before the introduction of the video assisted transurethral resection in the late 80 s, endoscopic procedures were performed by urologists bending over between the legs of the patient to have direct view on the operating field through a scope. ${ }^{2}$ Nowadays urological practices are equipped with rigid and flexible scopes, HD-camera's and movable monitors to perform their procedures.

Laparoscopy is a video assisted operation with long instrument that enters the patients' body via small incisions of approximately $1 \mathrm{~cm}$. Advantages of this form of surgery are among others: cosmetic benefits, less blood loss, less pain and shorter hospital stay. Surgeons' benefits, however, are less outspoken because they have to stand in a static position for a few hours.

Recent development in the management of urological diseases is robot assisted laparoscopy. This type of surgery offers significant advantages in terms of physical ergonomics for the surgeon who sits comfortably in front of the master console working with tool handles that offers more degrees of freedom, compared to conventional laparoscopic instruments.

All types of minimal invasive urology have a longer learning curve compared to open surgery as a result of longer instruments, less tactile feedback and limited workspace. ${ }^{3-5}$ In laparoscopy the tip of the instruments move opposite to the hand due to the fulcrum effect, e.g. when the urologist moves his hand to the right, the tip of the instrument will move left inside the patients' body. ${ }^{6}$ The latter ensures that hand-eye coordination and spatial awareness are of major importance.

At present-day the European working time directive (2004), ethical and financial considerations raise barriers to achieving appropriate levels of mastery through training in the operating theatre alone. ${ }^{7-9}$ Furthermore performing a first procedure directly on the patient is inacceptable nowadays as legal considerations are making high demands on surgeons' proficiency and standards of patient safety. ${ }^{10-12}$ Traditionally acquisition of skills in surgery is based on learning on patients and it is becoming increasingly apparent that the centuries-old teaching paradigm of the Halstadian model (1889), "see one, do one, teach one", is inappropriate in a world of rapidly introduced advanced technologies that require certain skills with high demands on quality and safety. ${ }^{12-15}$ Consequently there is a need for alternative forms of training to decrease errors and shorten learning curves. 


\section{Simulators}

For quite some time simulator based training has been advocated as an adjunct to surgical skills training. ${ }^{9,16}$ Key advantage of simulation-based training is that it allows learners to gain procedural skills in a safe setting outside the clinical area where they can make mistakes without doing harm to the patient. ${ }^{13,14,16}$

Research on simulator based skills education in urology started in the 1990s with the development of animate, box and virtual reality trainers. ${ }^{9}$ Animate training models comprises life animals, animal cadavers or parts of an animal suitable for training. However, disadvantage of an animal model is that the training has to confirm strict hygiene regulations, needs specialized skills laboratories with high skilled personnel and it is costly, as remains of the animal model cannot be reused for a second training. ${ }^{15}$

Box-trainers are cardboard or synthetic boxes with reusable exercises. Advantages of the box-trainer are the relatively low costs, the exercises are reusable many times and original instruments that are used in the operation theatre can be used for practice in the box-trainer. However a mentor is still needed to give instructions and feedback on the learners' performance. ${ }^{15}$

Virtual reality simulators (VR-simulator) are high-tech computerized simulators that vary in their ability to provided training from simple hand-eye exercises to whole procedure training programs. Engineers try to approximate real time haptic feedback and surgical instruments. Major advantage of a VR-simulator is that it can track learners' performances and that it provides feedback through numerical scores after each exercise. ${ }^{17}$, ${ }^{18}$ Main disadvantage is its costs to purchase a simulator, not to mention the annual costs for license and software updates. ${ }^{15}$

\section{Validity of training models}

Before a simulator can be implemented in a curriculum its effectiveness should be established, because it is important that the simulator meets its value as a skills trainer and to predict a learners' performance. In other words, the simulator needs to train what it is intended to train.

Effectiveness of a simulator can be researched by a systematically validation process. Different definitions of validity have been described. In this thesis we use the following definition of validity: "validity measures whether the simulator is actually teaching or evaluating what it is intended to teach or measure."19 The validation process comprises subjective validity (face and content) and objective validity (construct and criterion). Face validity assesses the opinions of non-experts and relates to the realism of the simulator. Content validity assesses the judgement of experts concerning the appropriateness of the simulator as a teaching modality. Construct validity measures the ability of the simulator to distinguish between novices and experts. It is obvious that the hypothesis 
is that the experts outperform the novices and that novices' performances improve with increasing experience in clinical practice.

The last step of the validation process is to measure criterion validity that consists of two types. The first type of criterion validity compares the results of the new simulator with an old technique or the gold standard (concurrent). The second type of criterion validity is the predictive validity that assesses to which extend the simulator predicts future performance. ${ }^{19}$

Various simulators have been subject of validation research in urology and it is evident that VR, synthetic and animal training models can be effective for surgical and urological residents to a certain extend. Only a few researchers managed to complete all steps of the validation process for a single simulator. For endourological procedures only the VRsimulator "Uro-mentor" completed the validation process for cystoscopy skills. ${ }^{20}$ Other endourological simulators for training TURP, TURBT and URS have proven face, content and construct validity, but some have not. ${ }^{20-33}$ Although the validation process is timeconsuming and costly, it remains of paramount importance that newly developed or modified simulators should be judged on its accurateness to train what it is intended to train before it is implemented in the curriculum.

Validation research for simulator based laparoscopic skills training was excessive in surgical field. The most extensively researched box-training was the Fundamentals of Laparoscopic Skills (FLS). The program consists of four theoretical modules via online access and a manual skills training based on the McGill Inanimate System for Training and Evaluation of Laparoscopic Skills (MISTELS) that consists of a box trainer offering five validated basic tasks. ${ }^{34-37}$ However, for laparoscopic urological skills, no specific laparoscopic training has been developed and it is not sure that the FLS tasks are all valid for urological practice.

\section{Skills assessment}

In response to urgent-calls from the government and the public for well-defined proficiency standards to safeguard the quality of care, the urological profession should accept its responsibility for patient safety and take the lead in setting proficiency standards." 14 But what is the definition of proficiency for a certain skill? Who is considered expert? And when is the right time for assessing proficiency in the residency curriculum? Traditionally proficiency in surgery has been verified by case-volume. However, the use of case-numbers to assess surgical competence is far from objective to evaluate technical skills. A high number of cases, does not automatically mean that those cases were performed well, with appropriate patient outcomes..$^{15,38}$

It is important to understand that the learning process from novice to expert is a continuum. According to Vygotsky's (1978) concept of a "zone of proximal development" each learner is on a progressive developmental pathway of expertise. In this concept the 
learner does not only acknowledge his capability of performance, but at some point he becomes aware how he can improve himself to raise to the expert level of competence. ${ }^{13}$ The framework of Dreyfus \& Dreyfus (1986) outlined five stages of progression from novice to expert where knowledge and skills move from basics with little situational perception in novices to the holistic intuitive approach of experts. ${ }^{13}$

Best-known framework of increasing levels of skill performance in medical education is "Millers triangle" (1990). The model claims that every novice starts with assimilating knowledge. In the second phase he "knows how" to apply that knowledge in certain skills. In the third phase the novice "shows" his acquired skills and in the last phase he performs the skills in clinical practice by "does". Every step of the pyramid can be used for an assessment moment with its specific testing methods. ${ }^{13}$

Two formats of assessment are described in literature: summative versus formative. Purpose of summative assessment is to come to a view of someone's' learning to measure his position in the learning process and/or for some form of certification. Formative assessment is more focusing on helping the learner in his progression with constructive feedback mechanisms. ${ }^{13}$

When putting all frameworks together it becomes apparent that when assessing learners one should take into account various factors, preferably integrating knowledge (cognition), psychomotor skills and level of competence. One of the challenges of developing a proficiency-based assessment in urology that has received little attention is how to establish pass-fail standards for the performance of skills.

\section{Designing simulator based training and assessment}

Surgical operations and urological procedures are considered complex tasks, since it encompasses psychomotor skills and cognitive decision-making skills. From the perspective of instructional design complex tasks are defined as those for which their performance require the integrated use of non-automated and automated knowledge and skills. ${ }^{39}$

Simulator based training is considered a promising modality to train parts of a complex urological procedure provided that it has proof of validity. However, a simulator that has proven validity will not automatically be incorporated in a curriculum. Schout et al ${ }^{24}$ recommended that a training program should be designed starting with a "training needs analysis" (TNA) to specify training objectives. The latter is described as the process of identifying the gap in training and related training needs. It is primarily conducted to determine where training is needed, what needs to be taught and who needs to be trained, including analysis of procedural steps and to identify the pitfalls of a procedure that needs to be trained. ${ }^{24,40}$ Subsequently it is important to identify what role the simulator can fulfill to train which part of the procedure and how the simulator based training can be integrated in the curriculum. Most difficult in analysis of training needs 
is filtering out expert experience to identify succeeding procedural steps and decisionmaking processes, and define for which part of the procedure simulator based training is most appropriate. Strategies from the field of instructional design could be useful to structure the training needs analysis for urological training programs.

\section{Human factors}

Minimal invasive urology confronts urologists with new challenges. One of the major changes compared to open surgery is that the urologist has been further removed from direct interaction with the patient tissue..$^{41}$ The science of human factors, so called ergonomics, analyses these challenges and formulate guidelines for creating a work environment that is safe for the surgeon and the operating team. Ergonomics is defined as "the scientific discipline concerned with the understanding of interactions among humans and other elements of a system, and the profession that applies theory, principles, data and methods to design in order to optimize human well-being and overall system performance" ${ }^{\prime \prime 2}$. Despite ergonomic guidelines for minimal invasive surgery, which deals with the placing of equipment in an ergonomic position and with ergonomic postures of the surgeon, awareness of these guidelines among surgeons is still marginal. ${ }^{43,44}$ Awareness of these ergonomic guidelines among urologists is still unknown.

\section{GENERAL PROBLEM DEFINITION}

In this thesis we focus on the development of simulator based training and assessment for minimal invasive urological procedures. Since minimal invasive urological techniques are rapidly emerging and volume-based learning in clinical practice is constraint, other training methods other than patient based are needed.

First, simulation-based training has been shown to be safe, time-efficient and cost-effective and for quite some time urological simulators have been validated. ${ }^{45,46}$ However not many of them have been integrated in the curriculum. It is obvious that development and validation of new simulators, or modification of validated simulators, should be continued to improve training ability. The question raises, which factors in the development process of simulator based training could narrow the gap between research and the current curriculum.

Second, hypothetically several simulators could be used for assessment purposes since they have proven discriminative ability. This assumption is never clearly established, as it has not yet been specified for which residency level simulator based assessment would be most appropriate and how to set competency standards.

Finally, minimal invasive urology is considered to be of major benefit for patients' cosmetics and post-operative recovery. Surgeons, however, still suffer from physical 
complaints due to laparoscopy despite extensive research on ergonomic improvement in the operation theatre. Insight in ergonomic complaints in urological practice had little attention in literature and there might be a potential for ergonomic attention in the field of simulator based training.

\section{The general research question of this thesis is:}

How can simulator based training and assessment be developed to improve learning in urology and to narrow the gap between research and integration in clinical practice? We formulated six specific research questions to address the general question:

\section{Research questions}

1. How useful is the newly developed Program for Laparoscopic Urological Skills (PLUS), which is based on the validated and widely used FLS-program (face and content validity)? Is the Program for Laparoscopic Urological Skills able to distinguish between those with different experience levels in laparoscopy (construct validity)?

2. Is the PLUS reliable as an assessment tool? How to set a tenable pass/fail standard for second year residents' minimal technical proficiency in basic laparoscopic skills?

3. What is the performance level of European urological residents according to the certification standards of the PLUS assessment?

4. What is the place of a newly developed TURP-simulator in the current urological curriculum according to a "training needs analysis"? How useful and realistic is the simulator and can it distinguish between novices' and experts' performances?

5. How to perform a "training needs analysis" for a complex procedural skill, the nephrostomy procedure, using the combined approach of cognitive task analysis and the 4-Component/Instructional Design (4C/ID) model?

6. Do urologists have physical complaints due to endourology and laparoscopy and how do urologists consider their knowledge about ergonomic conditions during minimal invasive urology? What is the preferred method of gaining knowledge about ergonomics according to urologists?

\section{OUTLINE OF THIS THESIS}

Chapter 2 focuses on the development of a suitable training program for learning basic laparoscopic urological skills that was based on the extensively validated FLS program. The modified program was renamed as "Program for laparoscopic urological skills" (PLUS). The PLUS was subordinated to a validation process to analyze whether the simulator meets usefulness and realism according to the opinions of novices, residents and urologists (face and construct validity). Subsequently we investigated whether it 
distinguishes between trainees with different experience levels in laparoscopy (construct validity). We sought evidence to support the hypothesis that the higher the level of laparoscopic experience, the shorter the time to complete a task, the higher the quality of task performance and the fewer errors.

To address the need for proficiency standards Chapter 3 shows the results of a study to set a proficiency level in the perspective of continuous learning. In the absence of a gold standard, there is no clearly preferred method and we conducted a study aimed at examining the reliability of the PLUS assessment and at setting a defensible pass/fail standard for second-year residents' minimal technical proficiency in basic laparoscopic skills. In Chapter 4 European level of basic laparoscopic skills proficiency is investigated using the certification standards of the PLUS assessment.

Chapter 5 evaluates the educational value of a virtual reality TURP simulator according to a curriculum-based approach. This chapter describes the results of a training needs analysis to identify procedural steps and pitfalls of the TURP-procedure. Subsequently it describes to what extent the simulator meets the results of the training needs analysis and whether it resembles realism and usefulness in the current curriculum according to the opinions of students, residents and urologists (face and content validity). We also determined the discriminative ability of the TURP simulator (construct validity). Sixty-six participants were involved in the validation process of the TURP simulator.

In Chapter $\mathbf{6}$ we analyzed the complex nephrostomy procedure by a structured method for training needs analysis from the perspective of instructional design. This chapter describes an approach combining cognitive task analysis and the 4C/ID model to create a blueprint for development of simulator based surgical training. Eight urologists participated in a semi-structured interview to analyze steps and sub steps of the nephrostomy procedure, and to identify which sub steps have reached a level of automaticity in experts.

Chapter $\mathbf{7}$ focuses on the results of an international survey of urologists' to investigate the frequency of musculoskeletal complaints among urologists who practice endourology and/or laparoscopy, how urologists' consider their knowledge about ergonomic conditions during minimal invasive urology and what their preferred method is for gaining knowledge about ergonomics. Participants were recruited at the annual congress of the European Association of Urology (EAU) and via e-mail. Two-hundred-and-eighty-five urologists responded to the questionnaire.

In Chapter 8 the findings from the studies reported in chapter 2-7 and future perspectives are summarized and discussed. 


\section{REFERENCES}

1. Blandy JP, Notley WG and Reynard JM. Transurethral resection. London: Taylor and Francis, 2005.

2. Widran J. Video transurethral resection using controlled continuous flow resectoscope. Urology. 1988; 31: 382-6.

3. Kumar U and Gill IS. Learning curve in human laparoscopic surgery. Curr Urol Rep. 2006; 7: 120-4.

4. Capelouto CC and Kavoussi LR. Complications of laparoscopic surgery. Urology. 1993; 42: 2-12.

5. Kerbl K and Clayman RV. Advances in laparoscopic renal and ureteral surgery. Eur Urol. 1994; 25: $1-6$.

6. Gallagher AG, McClure N, McGuigan J, Ritchie K and Sheehy NP. An ergonomic analysis of the fulcrum effect in the acquisition of endoscopic skills. Endoscopy. 1998; 30: 617-20.

7. Kelly BD, Curtin PD and Corcoran M. The effects of the European Working Time Directive on surgical training: the basic surgical trainee's perspective. Ir J Med Sci. 2011; 180: 435-7.

8. Parsons BA, Blencowe NS, Hollowood AD and Grant JR. Surgical training: the impact of changes in curriculum and experience. J Surg Educ. 2011; 68: 44-51.

9. Scott DJ. Patient safety, competency, and the future of surgical simulation. Simul Healthc. 2006; 1 : 164-70.

10. Shaw K, Cassel CK, Black C and Levinson W. Shared medical regulation in a time of increasing calls for accountability and transparency: comparison of recertification in the United States, Canada, and the United Kingdom. JAMA. 2009; 302: 2008-14.

11. Hout FA, Nienhuis ED, Robben PB, Frederiks BJ and Legemaate J. Supervision by the Dutch Healthcare Inspectorate. Eur J Health Law. 2010; 17: 347-60.

12. Kohn LT, Corrigan JM and Donaldson MS. To err is human: Building a safer health system. 7 ed. Washington DC: National Academy Press, 2008.

13. Dornan T, Mann K, Scherpbier AJ and Spencer J. Medical Education: Theory and Practice. London: Churchill Livingstone Elsevier, 2011.

14. Rodriguez-Paz JM, Kennedy M, Salas E, et al. Beyond "see one, do one, teach one": toward a different training paradigm. Qual Saf Health Care. 2009; 18: 63-8.

15. Reznick RK and MacRae H. Teaching surgical skills_changes in the wind. N Engl J Med. 2006; 355: 2664-9.

16. Issenberg SB, McGaghie WC, Hart IR, et al. Simulation technology for health care professional skills training and assessment. JAMA. 1999; 282: 861-6.

17. Verdaasdonk EG, Stassen LP, Monteny LJ and Dankelman J. Validation of a new basic virtual reality simulator for training of basic endoscopic skills: the SIMENDO. Surg Endosc. 2006; 20: 511-8.

18. Zhang A, Hunerbein M, Dai Y, Schlag PM and Beller S. Construct validity testing of a laparoscopic surgery simulator (Lap Mentor): evaluation of surgical skill with a virtual laparoscopic training simulator. Surg Endosc. 2008; 22: 1440-4.

19. McDougall EM. Validation of surgical simulators. J Endourol. 2007; 21:244-7.

20. Schout BM, Ananias HJ, Bemelmans BL, et al. Transfer of cysto-urethroscopy skills from a virtualreality simulator to the operating room: a randomized controlled trial. BJU Int. 2010; 106: 226-31; discussion 31.

21. Schout B, Dolmans V, Bemelmans B, Schoot D, Scherpbier A and Hendrikx A. Teaching diagnostic and therapeutic procedures of bladder pathology using a newly developed pig bladder model. J Endourol. 2008; 22: 2547-53. 
22. Schout BM, Bemelmans BL, Martens EJ, Scherpbier AJ and Hendrikx AJ. How useful and realistic is the uro trainer for training transurethral prostate and bladder tumor resection procedures? J Urol. 2009; 181: 1297-303; discussion 303.

23. Schout BM, Muijtjens AM, Hendrikx AJ, et al. Acquisition of flexible cystoscopy skills on a virtual reality simulator by experts and novices. BJU Int. 2010; 105: 234-9.

24. Schout BM, Hendrikx AJ, Scheele F, Bemelmans BL and Scherpbier AJ. Validation and implementation of surgical simulators: a critical review of present, past, and future. Surg Endosc. 2010; 24: 536-46.

25. Dolmans VE, Schout BM, de Beer NA, Bemelmans BL, Scherpbier AJ and Hendrikx AJ. The virtual reality endourologic simulator is realistic and useful for educational purposes. J Endourol. 2009; 23: $1175-81$.

26. Wijn RP, Persoon MC, Schout BM, Martens EJ, Scherpbier AJ and Hendrikx AJ. Virtual reality laparoscopic nephrectomy simulator is lacking in construct validity. J Endourol. 2010; 24: 117-22.

27. Mishra S, Kurien A, Ganpule A, Veeramani M, Sabnis RB and Desai M. Face and content validity of transurethral resection of prostate on Uro Trainer: is the simulation training useful? J Endourol. 2010; 24: 1839-43.

28. Sweet R, Kowalewski T, Oppenheimer P, Weghorst S and Satava R. Face, content and construct validity of the University of Washington virtual reality transurethral prostate resection trainer. J Urol. 2004; 172: 1953-7.

29. Sweet RM. Review of trainers for transurethral resection of the prostate skills. J Endourol. 2007; 21: 280-4.

30. Sweet RM, Hananel D and Lawrenz F. A unified approach to validation, reliability, and education study design for surgical technical skills training. Arch Surg. 2010; 145: 197-201.

31. Hudak SJ, Landt CL, Hernandez J and Soderdahl DW. External validation of a virtual reality transurethral resection of the prostate simulator. J Urol. 2010; 184: 2018-22.

32. Kallstrom R, Hjertberg $\mathrm{H}$, Kjolhede $\mathrm{H}$ and Svanvik J. Use of a virtual reality, real-time, simulation model for the training of urologists in transurethral resection of the prostate. Scand J Urol Nephrol. 2005; 39: 313-20.

33. Mishra S, Sharma R, Kumar A, Ganatra P, Sabnis RB and Desai MR. Comparative performance of high-fidelity training models for flexible ureteroscopy: Are all models effective? Indian J Urol. 2011; 27: 451-6.

34. Fraser SA, Klassen DR, Feldman LS, Ghitulescu GA, Stanbridge D and Fried GM. Evaluating laparoscopic skills: setting the pass/fail score for the MISTELS system. Surg Endosc. 2003; 17: 964-7.

35. Fried GM, Feldman LS, Vassiliou MC, et al. Proving the value of simulation in laparoscopic surgery. Ann Surg. 2004; 240: 518-25; discussion 25-8.

36. McCluney AL, Vassiliou MC, Kaneva PA, et al. FLS simulator performance predicts intraoperative laparoscopic skill. Surg Endosc. 2007; 21: 1991-5.

37. Peters JH, Fried GM, Swanstrom LL, et al. Development and validation of a comprehensive program of education and assessment of the basic fundamentals of laparoscopic surgery. Surgery. 2004; 135: 21-7.

38. Scott DJ, Valentine RJ, Bergen PC, et al. Evaluating surgical competency with the American Board of Surgery In-Training Examination, skill testing, and intraoperative assessment. Surgery. 2000; 128: 613-22.

39. Clark RE, Feldon DF, van Merrienboer JJ, Yates KA and Early S. Cognitive Task Analysis. In: al SJMe, (ed.). Handbook of Research on Educational Communications and Technology. London: Taylor and Francis Group, 2008, p. 577-93. 
40. Salas E and Cannon-Bowers JA. The science of training: a decade of progress. Annu Rev Psychol. 2001; 52: 471-99.

41. Berguer R. Surgery and ergonomics. Arch Surg. 1999; 134: 1011-6.

42. www.iea.nl.

43. van Det MJ, Meijerink WJ, Hoff C, Totte ER and Pierie JP. Optimal ergonomics for laparoscopic surgery in minimally invasive surgery suites: a review and guidelines. Surg Endosc. 2009; 23: 1279-85.

44. Wauben LS, van Veelen MA, Gossot D and Goossens RH. Application of ergonomic guidelines during minimally invasive surgery: a questionnaire survey of 284 surgeons. Surg Endosc. 2006; 20: $1268-74$

45. Ahmed K, Jawad M, Abboudi M, et al. Effectiveness of procedural simulation in urology: a systematic review. J Urol. 2011; 186: 26-34.

46. Gohil R, Khan RS, Ahmed K, et al. Urology training: past, present and future. BJU Int. 2012; 109: 1444-8.

47. Autorino R, Haber GP, Stein RJ, et al. Laparoscopic training in urology: critical analysis of current evidence. J Endourol. 2010; 24: 1377-90.

48. Bashankaev B, Baido S and Wexner SD. Review of available methods of simulation training to facilitate surgical education. Surg Endosc. 2011; 25: 28-35.

49. Dunkin B, Adrales GL, Apelgren $\mathrm{K}$ and Mellinger JD. Surgical simulation: a current review. Surg Endosc. 2007; 21: 357-66.

50. Scott DJ and Dunnington GL. The new ACS/APDS Skills Curriculum: moving the learning curve out of the operating room. Journal of gastrointestinal surgery : official journal of the Society for Surgery of the Alimentary Tract. 2008; 12: 213-21.

51. Al-Shaiji TF, Kanaroglou N, Thom A, et al. A cost-analysis comparison of laparoscopic radical prostatectomy versus open radical prostatectomy: the McMaster Institute of Urology experience. Canadian Urological Association journal=Journal de I'Association des urologues du Canada. 2010; 4: 237-41.

52. Coelho RF, Rocco B, Patel MB, et al. Retropubic, laparoscopic, and robot-assisted radical prostatectomy: a critical review of outcomes reported by high-volume centers. J Endourol. 2010; 24: 2003-15.

53. Eskicorapci SY, Teber D, Schulze M, Ates M, Stock C and Rassweiler JJ. Laparoscopic radical nephrectomy: the new gold standard surgical treatment for localized renal cell carcinoma. TheScientificWorldJournal. 2007; 7: 825-36.

54. Ficarra V, Novara G, Artibani W, et al. Retropubic, laparoscopic, and robot-assisted radical prostatectomy: a systematic review and cumulative analysis of comparative studies. Eur Urol. 2009; 55: 1037-63.

55. Hemal AK, Kumar A, Kumar R, Wadhwa P, Seth A and Gupta NP. Laparoscopic versus open radical nephrectomy for large renal tumors: a long-term prospective comparison. J Urol. 2007; 177: 8626.

56. Fowler DL. Enabling, implementing, and validating training methods in laparoscopic surgery. World journal of surgery. 2010; 34: 621-4.

57. Laguna MP, de Reijke TM and de la Rosette JJ. How far will simulators be involved into training? Curr Urol Rep. 2009; 10: 97-105.

58. Melvin WS, Johnson JA and Ellison EC. Laparoscopic skills enhancement. Am J Surg. 1996; 172: 377-9. 
59. Rassweiler J, Klein J, Teber D, Schulze M and Frede T. Mechanical simulators for training for laparoscopic surgery in urology. J Endourol. 2007; 21: 252-62.

60. Rosser JC, Rosser LE and Savalgi RS. Skill acquisition and assessment for laparoscopic surgery. Arch Surg. 1997; 132: 200-4.

61. Wignall GR, Denstedt JD, Preminger GM, et al. Surgical simulation: a urological perspective. J Urol. 2008; 179: 1690-9.

62. Kneebone R. Simulation, safety and surgery. Qual Saf Health Care. 2010; 19 Suppl 3: i47-52.

63. Peters JH, Fried GM, Swanstrom LL, et al. Development and validation of a comprehensive program of education and assessment of the basic fundamentals of laparoscopic surgery. Surgery. 2004; 135: 21-7.

64. Dauster B, Steinberg AP, Vassiliou MC, et al. Validity of the MISTELS simulator for laparoscopy training in urology. J Endourol. 2005; 19: 541-5.

65. Aggarwal R, Hance J, Undre S, et al. Training junior operative residents in laparoscopic suturing skills is feasible and efficacious. Surgery. 2006; 139: 729-34.

66. Moorthy K, Munz Y, Dosis A, Bello F, Chang A and Darzi A. Bimodal assessment of laparoscopic suturing skills: construct and concurrent validity. Surg Endosc. 2004; 18: 1608-12.

67. Hojat M and Xu G. A visitor's guide to effect sizes: statistical significance versus practical (clinical) importance of research findings. Advances in health sciences education : theory and practice. 2004; 9: 241-9.

68. Ericsson KA. Deliberate practice and the acquisition and maintenance of expert performance in medicine and related domains. Acad Med. 2004; 79: S70-81.

69. Ericsson KA. Deliberate practice and acquisition of expert performance: a general overview. Acad Emerg Med. 2008; 15: 988-94.

70. Wentink M, Stassen LP, Alwayn I, Hosman RJ and Stassen HG. Rasmussen's model of human behavior in laparoscopy training. Surg Endosc. 2003; 17: 1241-6.

71. Menhadji A, Abdelshehid C, Osann K, et al. Tracking and assessment of technical skills acquisition among urology residents for open, laparoscopic, and robotic skills over 4 years: is there a trend? J Endourol. 2013; 27: 783-9.

72. Berguer R. Surgical technology and the ergonomics of laparoscopic instruments. Surg Endosc. 1998; 12: 458-62.

73. Adams DM, Fenton SJ, Schirmer BD, Mahvi DM, Horvath K and Nichol P. One size does not fit all: current disposable laparoscopic devices do not fit the needs of female laparoscopic surgeons. Surg Endosc. 2008; 22: 2310-3.

74. Berguer R, Forkey DL and Smith WD. The effect of laparoscopic instrument working angle on surgeons' upper extremity workload. Surg Endosc. 2001; 15: 1027-9.

75. Berquer R, Smith WD and Davis S. An ergonomic study of the optimum operating table height for laparoscopic surgery. Surg Endosc. 2002; 16: 416-21.

76. Khan N, Abboudi H, Khan MS, Dasgupta P and Ahmed K. Measuring the surgical 'learning curve': methods, variables and competency. BJU Int. 2013; 113:504-8.

77. van Merrienboer JJ and Sweller J. Cognitive load theory in health professional education: design principles and strategies. Med Educ. 2010; 44: 85-93.

78. Grol R and Grimshaw J. Evidence-based implementation of evidence-based medicine. Jt Comm J Qual Improv. 1999; 25: 503-13.

79. Grol R and Wensing M. What drives change? Barriers to and incentives for achieving evidencebased practice. Med J Aust. 2004; 180: 57-60. 




\section{ABSTRACT}

\section{Objective}

To develop and evaluate a program for laparoscopic urological skills (PLUS) to determine the face, content and construct validation to achieve uniformity and standardization in training residents in urology.

\section{Methods}

The PLUS consists of five basic laparoscopic tasks. Three tasks were abstracted from the Fundamentals of Laparoscopy Surgery program, and 2 additional tasks were developed under continuous evaluation of expert urologists. Fifty participants were recruited from different hospitals and performed the final PLUS training. They all completed a questionnaire after performance. Three outcome parameters were measured: performance quality, time and dropped objects. The relationship between laparoscopic experience and the outcome parameters was investigated.

\section{Results}

Of the 50 participants, 13 were students, 20 were residents, and 17 were urologists. Double-log-linear regression for all 5 tasks showed a significant effect (effect size range 0.53-0.82; $\mathrm{P}<$.0005) for laparoscopic experience on performance time. Substantial correlations were found between experience and quality ratings (log linear regression effect size $0.37 ; \mathrm{P}=.012$ ) and the number of dropped objects (Spearman correlation effect size $0.49 ; \mathrm{P}<.01)$. The usefulness of the PLUS model as a training tool for basic laparoscopic skills was rated 4.55 on a scale from 1 (=not useful) to 5 (=useful) (standard deviation 0.58 ; range $3-5$ ).

\section{Conclusion}

The results of the present study indicated face, content and construct validity for the PLUS. The training is considered appropriate for use as a primary training tool for an entry test or as part of a step-wise training program in which basic and procedural laparoscopic skills are integrated. 


\section{INTRODUCTION}

With different forces eroding the feasibility of the conventional Halstedian model of "see one, do one, teach one", rapid changes are taking place in the teaching and learning of surgical skills. Ethical, legal and financial considerations raise barriers to achieving the appropriate levels of mastery through training in the operating theatre alone, thereby necessitating opportunities for new teaching and training methods. For quite some time, simulator based training has been advocated as a logical and promising method for surgical skills training. ${ }^{1-6}$

As minimal invasive surgery has become more important in urological practice, increased emphasis has been placed on laparoscopic education..$^{7-11}$ Laparoscopic surgery requires training in technical skills, such as hand eye coordination and spatial awareness, which are different from the skills needed for open procedures. ${ }^{3,12-17}$ Extensive studies have been undertaken to develop and evaluate simulators for minimally invasive techniques. ${ }^{18}$ In the United States, the Fundamentals of Laparoscopic Surgery (FLS) program, based on the McGill Inanimate System for Training and Evaluation of Laparoscopic Skills, was introduced to teach fundamental laparoscopic knowledge and skills and has been extensively validated. ${ }^{19-22}$ Dauster et $\mathrm{al}^{23}$ reported evidence of its construct validity for urology training in the United States, where training and certification are incorporated in the residency program. In Europe, training methods for minimally invasive techniques outside the operating theatre are being developed based on the FLS program and adapted to fit the European context. Because of these modifications, the adapted program must be validated before it can be implemented.

The aim of the present study was to examine the usefulness of a newly developed Program for Laparoscopic Urological Skills (PLUS), which is based on the validated and widely used FLS program (face validity and content validity). In addition, we investigated whether the PLUS was able to distinguish between those with different experience levels in laparoscopy (construct validity). We sought evidence to support the hypothesis that the greater the level of laparoscopic experience, the shorter the time to complete a task, the greater the quality of task performance, and the fewer errors.

\section{MATERIAL AND METHODS}

\section{Development of the PLUS}

We submitted the original FLS training program to the judgement of 8 Dutch urologists, all from different hospitals, who are considered experts in the field of urologic laparoscopy, having performed >500 laparoscopic procedures and/or taught (inter) national laparoscopy courses. After watching the theoretical modules on CD-ROM and 
performing the 5 original tasks on the box trainer, the experts expressed their opinions in a questionnaire considering usefulness of each task for urologic practice.

Three tasks received a positive evaluation from all urologists (peg transfer, pattern cutting and intracorporeal knot tying). However $5(63,5 \%)$ and $7(87,5 \%)$ of the 8 urologists considered the extracorporeal knot tying and the endoloop not relevant, respectively. Asked to suggest essential alternative tasks, the experts proposed a "needle guidance task" to train needle positioning and eye-hand coordination and a "clip-and-cut task" simulating clipping and cutting of the renal vessels during laparoscopic nephrectomy. After a thorough discussion, consensus on the exclusion of the 2 FLS tasks and inclusion of the 2 suggested tasks was reached in a meeting with the same experts. The 2 new tasks were then developed and continuously adjusted, in accordance with suggestions and comments from the same experts, in cooperation with a skills laboratory technician of Catharina Hospital, Eindhoven. The final PLUS training includes 5 tasks of increasing complexity (fig. 1).

The tasks were performed using the FLS box trainer, with a fixed-position video camera, 2 trocars with a fixed position, standard laparoscopic instruments (Karl Storz, Tuttlingen, Germany), a Hem-o-Lok clip applier (Teleflex Medical, Research Triangle Park. $\mathrm{NC}$ ) and a monitor. For the knot-tying and needle guidance task, a suture of Polysorb 3-0 (Covidien, Dublin, Ireland) was used.

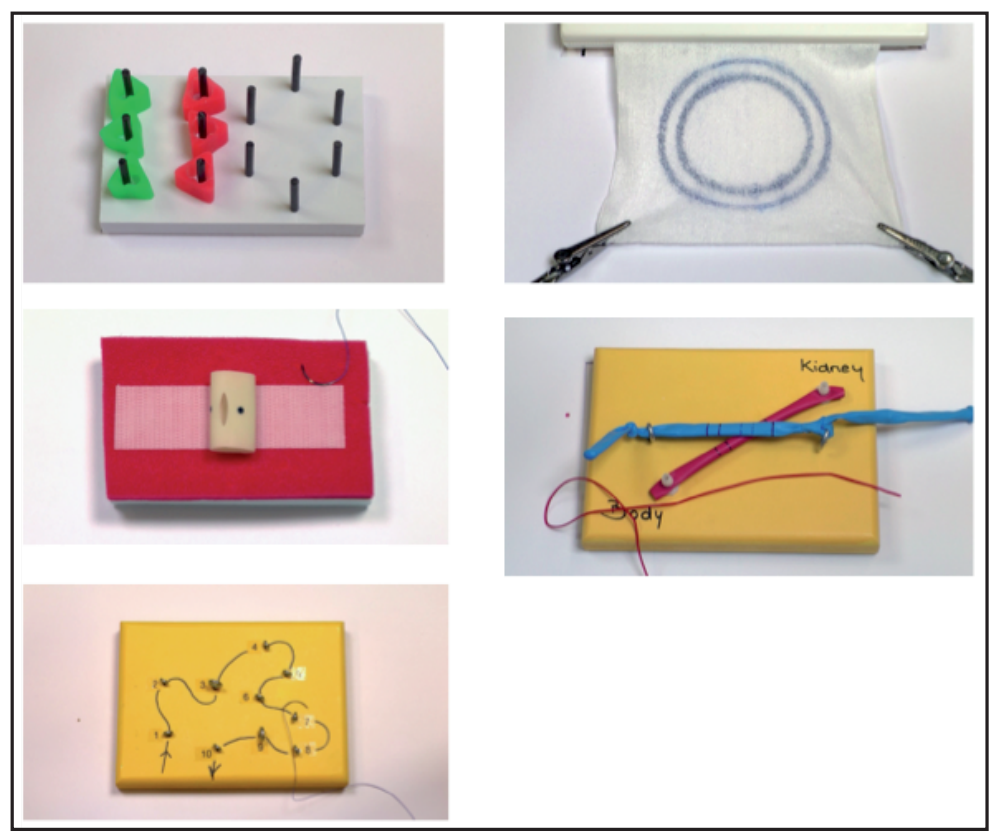

Figure 1. PLUS tasks: Peg transfer, cutting a circle, single knot tying, clip and cut, needle guidance 


\section{Validation}

In the present study, we validated the PLUS according to the standardized steps of the validation process described by McDougal et al. ${ }^{24}$ Face validity is defined as the "judgement of novices regarding usefulness of the simulator", and content validity is defined as the "judgement of experts regarding usefulness (appropriateness) of the simulator". Construct validity indicates whether the simulator is able to distinguish between experienced and inexperienced urologists ${ }^{18}$.

\section{Participants}

We recruited 50 participants from different hospitals and different levels of laparoscopic experience during laparoscopy courses and/or by electronic mail or by approaching them individually at their hospital. Included were urologists who perform laparoscopy, urology residents and senior medical students (year 3 or later). Because it was logistically not feasible to transfer all participants to 1 hospital, the tests were held during courses or in participants' own hospitals.

All participants provided informed consent. No ethical approval was required because no patients were involved and the test results did not have a substantial effect on direct patient care.

\section{Procedure}

After explaining the tasks according to a standardized protocol, the participants were given 1 minute of practice time for each task to become familiar with the instruments and task. For the actual test, the participants performed each task twice in succession (trials). All participants completed the PLUS tasks in the same order, from 1 to 5 . Their performance was measured by recording the "time to complete the task", the number of "'dropped objects" (task 1) and the "quality" of task performance (for task 2-4). The participants received no further guidance during the test.

For the first 2 tasks the FLS-protocol was used. Task 3 (single knot tying) was slightly little modified in the present study. We began timing when the needle was inserted in the rubber instead of the moment when the instruments were visible on the monitor. In the pilot study, it appeared that the students needed an excessive amount of time, up to 10 minutes, to position the needle in the needle driver before inserting it correctly in the rubber.

The following protocol was defined for the two newly developed tasks:

1. Clip-and-Cut: this exercise is a simplified representation of clipping and cutting the renal vessels during nephrectomy. The trainee is required to place a loop with traction around a blue tube or "renal vein" to visualize the red tube or "renal artery". The trainee then places 3 clips on the artery with a Hem-o-Lok before making the cut. 
The same procedure is repeated for the vein. Timing began when the participant touched the loop and stopped when the artery was cut.

2. Needle Guidance: the trainee was required to guide the needle through 10 metal rings following a set route. It is of no importance at which side the needle enters a ring. Timing began when the participant grasped the needle and stopped when the needle entered the last ring.

Performance was measured by recording the time with a stopwatch, the number of dropped objects in tasks 1 and the errors made in tasks 2-4.The time needed and dropped objects were recorded by a researcher. Two examiners, who were different from the researcher and unaware of the participants' names, gave their judgement on errors independently. To judge the quality, we used a binominal 14-item checklist consisting of several relevant parameters from an existing and validated checklist for laparoscopic suturing. ${ }^{25,26}$ For every error a score of 0 was applied.

After the test, the participants were given a questionnaire concerning their demographics and baseline laparoscopic experience, defined as the "number of laparoscopic procedures performed independently or under supervision". The participants were also given a questionnaire asking them to give their opinion with regard to the usefulness of the 2 new tasks (clip-and-cut and needle guidance) and of the box trainer in general on a five-point scale (1, not useful; 5 , very useful).

\section{Statistical Analysis}

Because we expected the distribution of the variables "time to complete the task" and "experience" to be substantially skewed to the right, we performed linear regression analysis of the transformed versions: $\log ($ Time) and log(Experience). This transformation was not required for the variables "dropped objects" and "quality", because their distributions were not skewed. To avoid the problem that log(Experience) would be undefined when participants report no experience, we used a slightly modified transformed value, $\log ($ Experience +0.1$)$. For this linear regression, with a single dependent variable, the correlation coefficient could be used as an indicator of effect size (ES). The ES indicates to what extent an effect is of practical (clinical) importance. ${ }^{27}$ To test the correlation between the number of dropped objects and experience, we used the Spearman's correlation coefficient. The Statistical Package for the Social sciences version 17 (SPSS, Chicago, IL) was used for all analyses. $\mathrm{P}<0.05$ was considered statistical significant. 


\section{RESULTS}

A total of 50 participants were included in the study: 13 students from the University of Maastricht, 20 urologic residents from 6 different hospitals, and 17 urologists from 10 different hospitals. The mean score for laparoscopic experience were 0 for students, $31 \pm 18$ for residents and $439 \pm 480$ for urologists. Five participants were excluded from the analysis of construct validity, because they did not complete the whole PLUS. One participant was excluded in analysis of face validity because he did not complete the final questionnaire.

\section{Face and content validity}

The participants' perceptions of the usefulness PLUS for training are listed in Table 1.

Of all participants, $92 \%$ supported the inclusion of the clip-and-cut task, although $21 \%$ of the residents indicated that the loop element in this task offered no additional value. Also, $96 \%$ percent supported inclusion of the needle guidance task. No significant differences were found among students, residents and urologists with regard to the perceived usefulness of the PLUS trainer ( $P=.137$, Kruskal Wallis Test).

Table 1. Opinions of all participants about usefulness of the PLUS and the 2 newly developed tasks on a scale from 1 to 5

\begin{tabular}{|c|c|c|}
\hline 1 = not useful; 5 =very useful & Mean \pm SD & Range \\
\hline \multicolumn{3}{|l|}{ Clip-and-Cut task } \\
\hline Hand-eye coordination & $4.33 \pm 0.72$ & $3-5$ \\
\hline Instrument Handling & $4.53 \pm 0.58$ & $3-5$ \\
\hline \multicolumn{3}{|l|}{ Needle Guidance task } \\
\hline Hand-eye coordination & $4.73 \pm 0.45$ & $4-5$ \\
\hline Instrument Handling & $4.66 \pm 0.52$ & $3-5$ \\
\hline \multicolumn{3}{|l|}{ PLUS } \\
\hline Hand-eye coordination & $4.70 \pm 0.50$ & $3-5$ \\
\hline Instrument Handling & $4.16 \pm 0.80$ & $2-5$ \\
\hline 3D-orientation & $4.27 \pm 0.86$ & $2-5$ \\
\hline $\begin{array}{l}\text { Overall score on usefulness of the simulator as an educational } \\
\text { tool }\end{array}$ & $4.55 \pm 0.58$ & $3-5$ \\
\hline
\end{tabular}

\section{Construct validity}

Substantial correlations were found between experience and time (double-log linear regression analysis, $\mathrm{ES}=0.55$ to $0.82 ; \mathrm{P}<.0005 ;$ Fig. 2). The regression-coefficient, correlation coefficient (ES), and $\mathrm{R}^{2}$ per task are listed in Table 2 . The decrease in time for experts 


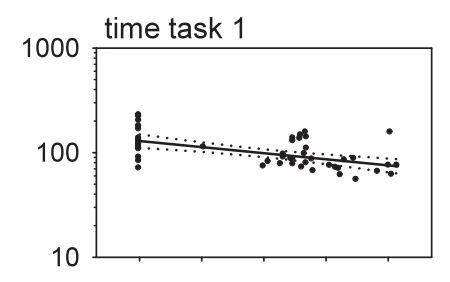

time task 2
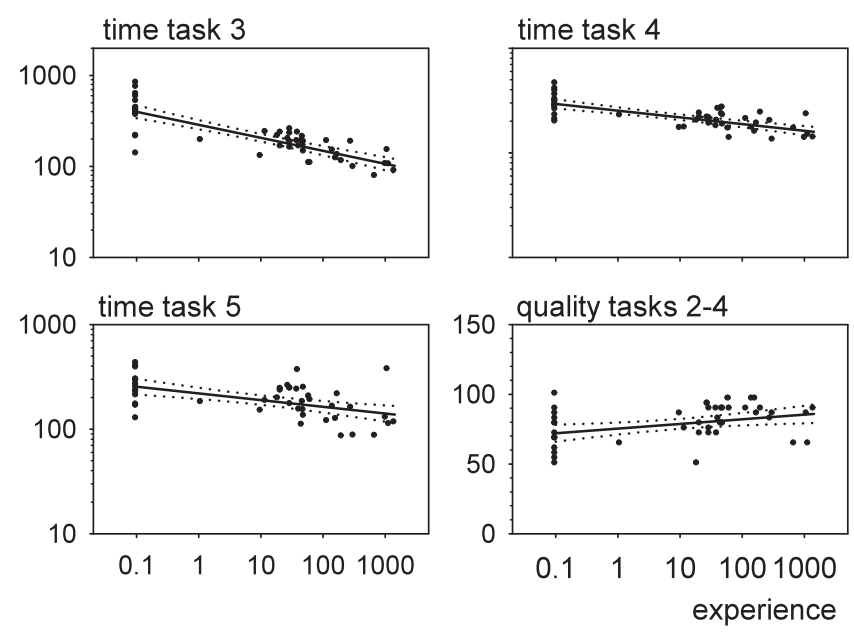

Figure 2. Linear regression analysis for "performance time versus experience" and "quality versus experience". Time task 1, peg transfer; time task 2, cutting gauze; time task 3, single knot tying; time task 4, clipping and cutting; time task 5, needle guidance. Logarithmic scale used for time and experience; $95 \%$ confidential intervals indicated by dotted lines and each tick dot indicates a participant.

versus novices (experience $=1000$ versus experience $=0.1$ ) ranged from $41 \%$ for tasks 1 and 2, 43\% for task 5 (needle guidance), $46 \%$ for task 4 (clip-and-cut) to $74 \%$ for task 3 (single knot tying). For tasks 1, 4 and 5 consistent learning effects were found, with a decrease in time from the first to the second trial of 13\% (task 1), 21\% (task 4) and 11\% (task 5). For tasks 2 and 3 similar effects were found for novices (22\% and 31\% decrease, respectively), but not for experts, whose time to completion showed an increase of $8 \%$ for task 2 and a decrease of 1\% for task 3.

A significant correlation was also found between experience and the quality of performance ratings (single-log linear regression analysis, $E S=0.37 ; P=.012$ ). For the quality ratings, a relative increase of $18 \%$ was found for experts versus novices (Fig. 2).

Finally, a significant correlation was found between the level of experience and the number of objects dropped during task 1 (Spearman correlation coefficient, ES=0.49; $\mathrm{P}$ $<.01$ ), where participants with more experience made fewer errors in terms of dropped objects. 
Table 2. Linear regression analysis of "time versus experience" and "quality versus experience"

\begin{tabular}{lcccc}
\hline & \multicolumn{2}{c}{ Regression coefficient (b) } & & \\
Time & $\mathrm{b}$ & $\begin{array}{c}\text { Relative decrease of Time From } \\
\text { Novice to Expert (\%) }\end{array}$ & Correlation coefficient (Effect size) & $\mathrm{R}^{2}$ \\
\hline T1 & $-0.058^{*}$ & 42 & $-0.55^{*}$ & 0.30 \\
T2 & $-0.057^{*}$ & 41 & $-0.55^{*}$ & 0.30 \\
T3 & $-0.143^{*}$ & 73 & $-0.82^{*}$ & 0.68 \\
T4 & $-0.064^{*}$ & 45 & $-0.73^{*}$ & 0.54 \\
T5 & $-0.064^{*}$ & 45 & $-0.53^{*}$ & 0.28 \\
PLUS quality & $3.29^{\dagger}$ & 18 & $0.37^{\dagger}$ & 0.14 \\
\hline
\end{tabular}

T. task; ${ }^{*} \mathrm{P}<.0005 ;{ }^{\dagger} \mathrm{P}=.012$

\section{COMMENT}

The results of the present study have confirmed the face, content and construct validity of a newly developed educational program, the PLUS, consisting of 5 basic tasks. Three tasks were abstracted from the FLS and 2 tasks were newly developed to fit the requirements of urology training programs.

\section{Face and content validity}

For establishing face and content validity, we based our cutoff point on previous studies by Sweet et $\mathrm{a}^{28}$ and Schout et al ${ }^{29}$. A cut-off point of 3 on a 5 -point scale $(0$, not useful; 5 , very useful) was used to determine the acceptability of the PLUS trainer. All ratings were greater than 3.5 and those for the needle guidance were consistently $>4.5$, suggesting that the PLUS can be considered a useful training method for laparoscopic urological skills with acceptable face and content validity.

The clip-and-cut task was introduced to learn how to clip and cut the renal vessels.

In particular for junior residents, who are not familiar with the Hem-o-Lok, it is considered of vital importance to learn the basics of this procedure. However, $21 \%$ of the residents thought the looping element should not be included in the task, because the traction given on the plastic tube was considered too hard and not realistic compared with the actual live traction with a loop on a renal vessel. However, all the urologists were in favor of inclusion of the looping element. This discordance of opinions suggests that this element should be evaluated carefully and possibly improved when the PLUS trainer is refined further. For example using a longer loop would obviate the need for hard traction.

An important suggestion for additional improvement was routing directions for the needle guidance task, preferably guided through the rings by the left and right instru- 
ment alternately. We will take this into consideration at further refinement of the PLUS tasks.

The overall rating of the PLUS trainer was $>4$, with 3-dimensional orientation the only aspect to receive lower rating owing to the fixed camera position. Finally, some participants remarked that the model was rather basic, because once the threshold performance criteria have been attained, no further improvement by the participant is possible.

\section{Construct Validity}

We used a different approach for establishing construct validity than the conventionally to define the difference between the novice and expert. We did so, because the generally used broad qualification of expert or non-expert in surgical skills and knowledge seems rather arbitrary and lacks the precision required for research. To avoid arbitrariness, we used the specific, continuous variable "experience," expressed as the number of laparoscopic operations performed and correlated this with the performance of basic laparoscopic tasks.

The strong correlation between "experience" and "time to complete the task" ( $\mathrm{P}<$ .0005) and "quality" ( $P=.012)$ for all five tasks indicates overall better performance for more experienced participants, a finding that confirms our hypothesis and supports the construct validity of the PLUS trainer.

However, experience was not the only predictor of performance. Only $30 \%$ of the variance in outcome with regard to time for task 1 (peg transfer) could be explained by experience, leaving $70 \%$ to be explained by other variables such as distraction, stress by time pressure, knowledge how to handle the instruments correctly, and so forth. However, for single knot tying and the clip-and-cut task, experience explained $76 \%$ and $50 \%$ of the time results, respectively. This suggests that experience is a better predictor of performance with regard to the more applied or advanced skills, which seems logical, because more advanced skills offer more scope for improvement by training.

\section{Limitations and future research}

One limitation might relate to the definition of experience we used. We are aware of the probability that the total number of laparoscopic procedures (surgical and urological) performed under supervision or independently was not accurately estimated. However, it can be assumed that the numbers given by the residents were accurate, because residents must record the number and specifications of the performed procedures in their portfolio. For the urologists the number was estimated, especially for those who had performed $>500$ procedures.

Second, students' opinions on the "usefulness of the PLUS trainer" should be interpreted with caution, because they had no relevant experience in laparoscopy; thus, their 
ratings might not be representative. In general, we have taken into account a certain amount of volunteer bias. All participants were willingly to perform the PLUS, which could imply that they already had a more positive attitude towards the PLUS than others who find laparoscopy less interesting.

Third, the relative increase of quality from novice to expert was only $18 \%$, which might have been because we assessed errors after task performance (assessment of the materials) and not during the actual performance. However, inter-rater reliability of this tool should still be established.

Finally, the results also suggest a substantial learning effect, especially for trainees with limited laparoscopic experience, although the learning effect seemed to vanish for highly experienced participants. However, no firm conclusions can be drawn with regard to individual learning curves on the basis of only two trials.

It would be worthwhile to focus future research on the learning curve within an individual and to identify the best training interval for the most efficient learning curve. The research protocol should not only focus on the amount of training, it should also consider aspects that might affect the efficiency of learning, such as different training intervals and the amount of mentoring. Furthermore, criterion validity of the PLUS trainer remains to be established, and a study should be conducted to determine the predictive value of PLUS performance for the performance of real time laparoscopy. However, it is not recommended to transfer these basic skills directly on patients but to use the animal laboratory as an alternative. Currently, using procedural simulators as the reference standard is not optimal, because none of the simulators of that type have shown criterion validity for urological laparoscopic procedures.

We recommend studying next steps in training laparoscopic skills, using a variety of simulators, such as virtual reality simulators and live animal or cadaver models, to develop an educational laparoscopy curriculum. In addition, the reliability of the test should be researched and standards set to define a threshold of acceptable performance for use in the assessment and maintenance of basic laparoscopic skills.

\section{CONCLUSIONS}

In the present study we showed evidence for face, content and construct validity of PLUS training to learn basic laparoscopic urological skills. The PLUS shows promise as a primary training tool for a step-wise training method in which different aspects of basic and procedural skills are integrated. Additional studies should investigate the next steps for laparoscopic training. Also, additional research is needed to define the standards of acceptable performance that can be used for assessment or maintenance of basic laparoscopic skills. 


\section{Acknowledgement}

To the 8 experts from Erasmus Medical Centre, Radboud Universitiy Nijmegen Medical Centre, Canisius-Wilhelmina Hospital, Jeroen Bosch Hospital, Scheperhospital, StFranciscus Gasthuis, St-Jansdal Hospital, Rijnstate Hospital, who participated in the development of the PLUS trainer; to all the medical students from Maastricht University and the residents in urology and urologists who were willing to participate in this validation study; and to Mereke Gorsira for her editorial assistance, with thanks to all. 


\section{REFERENCES}

1. Autorino R, Haber GP, Stein RJ, et al. Laparoscopic training in urology: critical analysis of current evidence. J Endourol. 2010; 24: 1377-90.

2. Bashankaev B, Baido S and Wexner SD. Review of available methods of simulation training to facilitate surgical education. Surg Endosc. 2011; 25: 28-35.

3. Dunkin B, Adrales GL, Apelgren K and Mellinger JD. Surgical simulation: a current review. Surg Endosc. 2007; 21: 357-66.

4. Reznick RK and MacRae H. Teaching surgical skills_changes in the wind. N Engl J Med. 2006; 355: 2664-9.

5. Schout BM, Hendrikx AJ, Scheele F, Bemelmans BL and Scherpbier AJ. Validation and implementation of surgical simulators: a critical review of present, past, and future. Surg Endosc. 2010; 24: 536-46.

6. Scott DJ and Dunnington GL. The new ACS/APDS Skills Curriculum: moving the learning curve out of the operating room. J of gastrointest surg. 2008; 12: 213-21.

7. Al-Shaiji TF, Kanaroglou N, Thom A, et al. A cost-analysis comparison of laparoscopic radical prostatectomy versus open radical prostatectomy: the McMaster Institute of Urology experience. Can Urol Assoc J. 2010; 4: 237-41.

8. Coelho RF, Rocco B, Patel MB, et al. Retropubic, laparoscopic, and robot-assisted radical prostatectomy: a critical review of outcomes reported by high-volume centers. J Endourol. 2010; 24: 2003-15.

9. Eskicorapci SY, Teber D, Schulze M, Ates M, Stock C and Rassweiler JJ. Laparoscopic radical nephrectomy: the new gold standard surgical treatment for localized renal cell carcinoma. TheScientificWorldJournal. 2007; 7: 825-36.

10. Ficarra V, Novara G, Artibani W, et al. Retropubic, laparoscopic, and robot-assisted radical prostatectomy: a systematic review and cumulative analysis of comparative studies. Eur Urol. 2009; 55: 1037-63.

11. Hemal AK, Kumar A, Kumar R, Wadhwa P, Seth A and Gupta NP. Laparoscopic versus open radical nephrectomy for large renal tumors: a long-term prospective comparison. J Urol. 2007; 177: 8626.

12. Fowler DL. Enabling, implementing, and validating training methods in laparoscopic surgery. World journal of surgery. 2010; 34: 621-4.

13. Laguna MP, de Reijke TM and de la Rosette JJ. How far will simulators be involved into training? Curr Urol Rep. 2009; 10: 97-105.

14. Melvin WS, Johnson JA and Ellison EC. Laparoscopic skills enhancement. Am J Surg. 1996; 172: 377-9.

15. Rassweiler J, Klein J, Teber D, Schulze M and Frede T. Mechanical simulators for training for laparoscopic surgery in urology. J Endourol. 2007; 21: 252-62.

16. Rosser JC, Rosser LE and Savalgi RS. Skill acquisition and assessment for laparoscopic surgery. Arch Surg. 1997; 132: 200-4.

17. Wignall GR, Denstedt JD, Preminger GM, et al. Surgical simulation: a urological perspective. J Urol. 2008; 179: 1690-9.

18. Kneebone R. Simulation, safety and surgery. Qual Saf Health Care. 2010; 19 Suppl 3: i47-52.

19. Fraser SA, Klassen DR, Feldman LS, Ghitulescu GA, Stanbridge D and Fried GM. Evaluating laparoscopic skills: setting the pass/fail score for the MISTELS system. Surg Endosc. 2003; 17: 964-7. 
20. Fried GM, Feldman LS, Vassiliou MC, et al. Proving the value of simulation in laparoscopic surgery. Ann Surg. 2004; 240: 518-25.

21. McCluney AL, Vassiliou MC, Kaneva PA, et al. FLS simulator performance predicts intraoperative laparoscopic skill. Surg Endosc. 2007; 21: 1991-5.

22. Peters JH, Fried GM, Swanstrom LL, et al. Development and validation of a comprehensive program of education and assessment of the basic fundamentals of laparoscopic surgery. Surgery. 2004; 135: 21-7.

23. Dauster B, Steinberg AP, Vassiliou MC, et al. Validity of the MISTELS simulator for laparoscopy training in urology. J Endourol. 2005; 19: 541-5.

24. McDougall EM. Validation of surgical simulators. J Endourol. 2007; 21: 244-7.

25. Aggarwal R, Hance J, Undre S, et al. Training junior operative residents in laparoscopic suturing skills is feasible and efficacious. Surgery. 2006; 139: 729-34.

26. Moorthy K, Munz Y, Dosis A, Bello F, Chang A and Darzi A. Bimodal assessment of laparoscopic suturing skills: construct and concurrent validity. Surg Endosc. 2004; 18: 1608-12.

27. Hojat M and Xu G. A visitor's guide to effect sizes: statistical significance versus practical (clinical) importance of research findings. Advances in health sciences education : theory and practice. 2004; 9: 241-9.

28. Sweet R, Kowalewski T, Oppenheimer P, Weghorst S and Satava R. Face, content and construct validity of the University of Washington virtual reality transurethral prostate resection trainer. J Urol. 2004; 172: 1953-7.

29. Schout BM, Bemelmans BL, Martens EJ, Scherpbier AJ and Hendrikx AJ. How useful and realistic is the uro trainer for training transurethral prostate and bladder tumor resection procedures? J Urol. 2009; 181: 1297-303. 



\section{ABSTRACT}

\section{Aim}

There is growing pressure from government and the public to define proficiency standards for surgical skills. Aim of this study was to estimate the reliability of the Program for Laparoscopic Urological Skills (PLUS) assessment and to set a certification standard for second-year urological residents.

\section{Methods}

Fifty participants were assessed on performance time and performance quality to investigate the reliability of the PLUS assessment. Generalisability coefficient of 0.8 , on a scale of 0 to 1.0, was considered to indicate good reliability for assessment purposes. Pass/fail standards were based on laparoscopic experience: novices, intermediates, and experts (>100 procedures). The pass/fail standards were investigated for the PLUS performances of 33 second-year urological residents.

\section{Results}

Fifteen novices, twenty-three intermediates and twelve experts were included. An intertrial reliability of $>0.80$ was reached with two trials for each task. Inter-rater reliability of the quality measurements was 0.79 for two judges. Pass/fail scores were determined for the novice/intermediate boundary and the intermediate/expert boundary. Pass rates for second-year residents were $63.64 \%$ and $9.09 \%$, respectively.

\section{Conclusion}

The PLUS assessment is reliable for setting a certification standard for second-year urological residents that serves as a starting point for residents to proceed to the next level of laparoscopic competency. 


\section{INTRODUCTION}

Since the introduction of laparoscopic nephrectomy in the early 1990s, laparoscopy has become an established technique in urological practice. ${ }^{1}$ Because laparoscopy requires other skills than open surgery, simulator based skills training is currently widely accepted. ${ }^{2,3}$ However, we are still seeking optimal methods for assessing laparoscopic skills performance. ${ }^{4-6}$ In response to urgent calls from government and the public for well-defined proficiency standards to safeguard the quality of care, the profession itself should accept its primary responsibility for patient safety and take the lead in setting proficiency standards based on professionals' knowledge and experience..$^{7-9}$

In an earlier project, we developed and evaluated the Program for Laparoscopic Urological Skills (PLUS), with training tasks partly adopted from the Fundamentals of Laparoscopic Surgery (FLS) and partly newly developed for urological purposes. The extensively validated FLS program comprises five basic tasks to teach fundamental laparoscopic skills. In an expert meeting with urologists, two of the five tasks (endo-loop and extracorporeal knot-tying) were considered not relevant for urological laparoscopic purposes. Therefore two new tasks were suggested and developed instead, a clip-andcut task simulating clipping and cutting the renal vessels in a laparoscopic nephrectomy and a needle-guidance task to train needle positioning. Since the original FLS program has been revised, the new program was renamed as PLUS.

Subsequently we validated the PLUS for the urological curriculum, according to the definitions of face, content and construct validity defined by McDougal et al. ${ }^{6}$ Face and content validity are defined as the judgement of novices and experts respectively, regarding usefulness of the simulator. Construct validity indicates whether the simulator is able to discriminate between novices and expert urologists. ${ }^{6,10}$ Thirteen students, 20 residents and 17 urologists were included. Usefulness of the PLUS as a training tool in the urological curriculum was rated 4.55 on a scale from 1 (=not useful) to 5 (useful) (SD 0.58; range 3-5). Double-log linear regression showed a significant effect (Effect Size range $0.53-0.83 ; \mathrm{p}<0.0005$ ) of laparoscopic experience and time and substantial correlations were found between experience and quality ratings (log-linear regression ES 0.37; $p=0.012$ ).

By showing face, content, and construct validity, this study supported the PLUS as a promising training tool for the first level of a stepwise laparoscopy curriculum. Minimal proficiency standards for PLUS assessment purposes, to filter out the truly non-competent trainee, remained to be established, however.

The ability of assessment to discriminate between competent and non-competent examinee performance is conditional on accurate performance standards ${ }^{11-14}$, depending on valid (measuring relevant outcomes) and reliable (replicable) assessment methods. 
In the absence of a gold standard, there is no clearly preferred method and any method will have some degree of arbitrariness. Arbitrariness can be reduced by determining the most appropriate, credible, and tenable assessment method. Simply setting a standard at a traditionally used mastery level (e.g. 60\%) is the most common, but at the same time the least tenable approach. ${ }^{15}$

In order to promote standardization early in the urological curriculum, we conducted a study aimed at

- examining the reliability of the PLUS assessment and at

- setting a tenable pass/fail standard for second-year residents' minimal technical proficiency in basic laparoscopic skills.

\section{MATERIALS AND METHODS}

We used identical materials and identical study groups comprising different levels of laparoscopic experience to examine the reliability of the PLUS and to set pass/fail standards based on experience levels. The consequences of different pass/fail standards for the PLUS performances of second-year urological residents were investigated. No ethical approval was required according to the medical ethical committee of our hospital, as there was no involvement of patients and the test result did not have substantial effect on direct patient care.

\section{Materials}

The PLUS assessment comprises five basic laparoscopic tasks in a box trainer of the Fundamentals of Laparoscopic Surgery (FLS) with a fixed camera position. With these tasks bimanual dexterity, hand-eye coordination, spatial awareness, suture technique and clipping and cutting skills are assessed. Identical boxes, tasks, suture material (Polysorb ${ }^{\circ} 3-0$, Tyco Healthcare, Mansfield, MA, USA), Hem-o-lok appliers (Teleflex Medical', Research Triagle Park, Durham, NC, USA) and laparoscopic instruments (Storz ${ }^{\circ}$ Tuttlingen, Germany) were used by all participants in the study. The first task required two dissectors, the second task a dissector and scissors, the third task two needle holders, the fourth task two dissectors, scissors and a Hem-o-Lok applier and finally the fifth task required two needle holders.

\section{Methods: Reliability of the PLUS assessment}

Between September and December 2009, 50 participants - medical students, urological residents, and laparoscopic urologists - were included in the study to test the reliability of the PLUS assessment. They were approached during urological courses and 


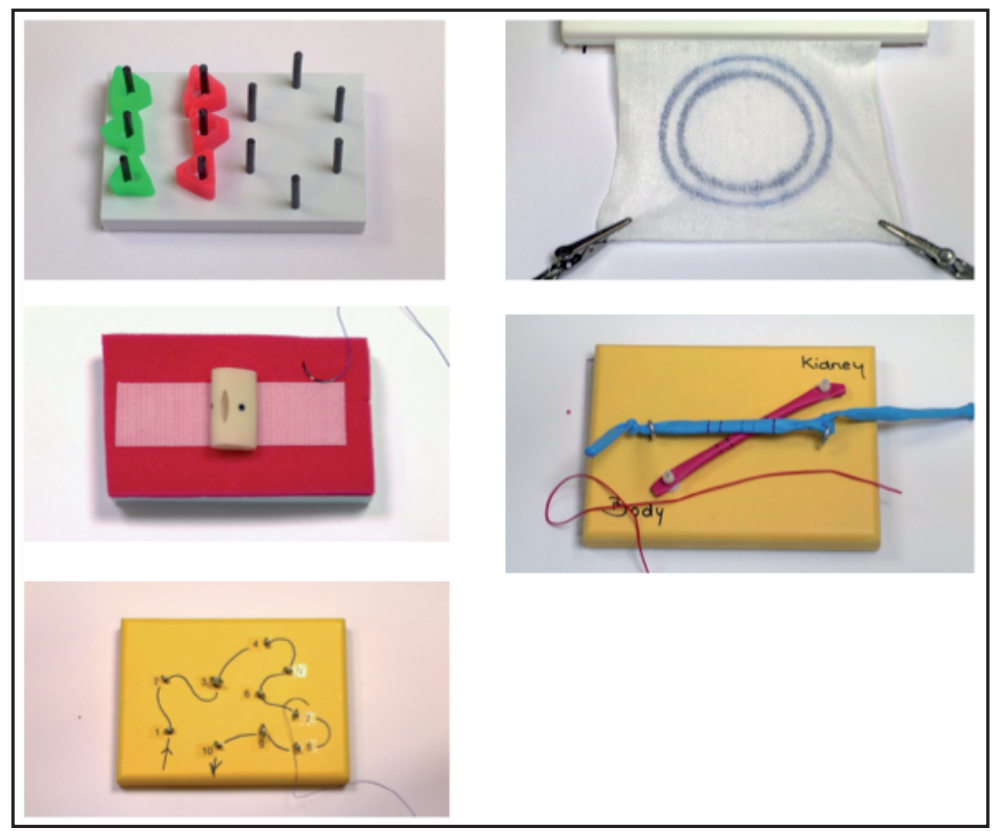

Figure 1. PLUS tasks: Peg transfer, cutting a circle, single knot tying, clip and cut, needle guidance

in six different hospitals in the Netherlands. All participants gave informed consent for inclusion in the study.

The participants received instructions according to a standardized protocol, followed by one minute practice time for each task in order to ascertain if they had understood correctly how the tasks were to be performed. Next, all participants performed each PLUS test task twice in succession (trials 1 and 2). No further guidance was given during the test. After the test, the participants completed a questionnaire regarding demographics and laparoscopic experience.

The assessment of task performance was based on the 'time to complete the task' and on the 'quality of the finished products' (tasks $2-4$ ). The principal researcher recorded

Table 1. Intra-performer reliability (Generalizability Coefficient) per task

\begin{tabular}{cccccc}
\hline & \multicolumn{5}{c}{ Number of trials per task } \\
\cline { 2 - 6 } & 1 & 2 & 3 & 4 & 0.98 \\
\hline Task 1 & 0.91 & 0.95 & 0.97 & 0.98 & 0.92 \\
Task 2 & 0.96 & 0.81 & 0.87 & 0.90 & 0.95 \\
Task 3 & 0.78 & 0.88 & 0.91 & 0.93 & 0.91 \\
Task 4 & 0.68 & 0.81 & 0.86 & 0.89 & 0.91 \\
Task 5 & 0.67 & 0.80 & 0.86 & 0.89 & \\
\hline
\end{tabular}


the performance time for each task using a stopwatch, and two urologists, blinded to the participants' names, independently scored the quality of task performances retrospectively on a binomial checklist partly derived from a validated checklist for laparoscopic suturing. ${ }^{16,17}$ Quality criteria included 'cut between the 2 lines' (task 2); 'knot is placed within $1 \mathrm{~mm}$ of the black dot','knot does not slip' and 'knot approximates the tissue' (task $3) ;$ ' 6 clips are placed on the tubes,' 'all clips are placed on the continuous lines' and 'all cuts are placed between the dotted lines' (task 4). For every correct performance, one point was assigned. The maximum score for the quality assessment after two trials was. ${ }^{14}$

The consistency of the tasks was examined by estimating inter-trial reliability using the outcome parameter 'time' over two trials per task. The results of the two examiners were compared to estimate inter-rater reliability.

Table 2. Inter-rater reliability (Generalizability Coefficient) for quality of performance when different numbers of raters are used

\begin{tabular}{lccccc}
\hline & \multicolumn{5}{c}{ Number of raters } \\
\cline { 2 - 6 } & 1 & 2 & 3 & 4 & 5 \\
\hline $\begin{array}{l}\text { Quality of } \\
\text { performance }\end{array}$ & 0.65 & $\mathbf{0 . 7 9}$ & 0.85 & 0.88 & 0.90 \\
\hline
\end{tabular}

\section{Methods: Setting a certification standard for second-year residents in urology}

The generalized examinee-centered method, described by Cohen et al., ${ }^{18}$ was used to establish the pass/fail standard for the PLUS performances of the 33 second-year residents. This educational approach uses the linear relationship between assessment scores and degree of procedural experience of multiple reference groups. Assessmentscores on the boundaries between different categories or groups are then used as pass/ fail criteria.

In this study, we classified the fifty participants, previously described, into three categories, based on laparoscopic experience defined as the number of procedures performed independently or under supervision: novices (0), intermediates (1 - 100), and experts $(>100)$.

The cut scores in the present study were based on the performances of these groups and we evaluated the second-year residents' pass rates for each task on the boundaries between novice/intermediate and intermediate/expert levels (figure 2).

Thirty-three second-year urological residents took the assessment in December 2009 $(\mathrm{N}=14)$ and December $2010(\mathrm{~N}=19)$, immediately after their surgical rotation, which included some laparoscopic experience. All residents gave informed consent for their inclusion in the study. 


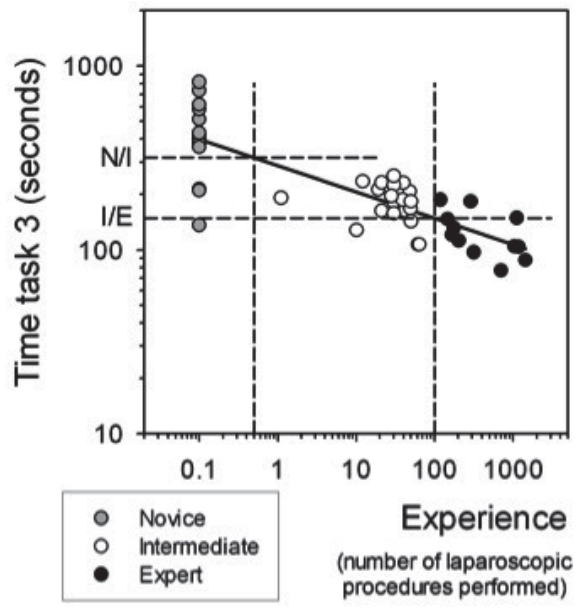

Figure 2. Illustrative model of the 'Generalized Examinee-Centered method' to set assessment standards at the boundaries of Novice/Intermediate (N/I) and Intermediate/Expert (I/E). Each circle represents a persons' time to complete the task' for task 3 (mean of two trials) versus experience. Vertical dashed lines indicates the $\mathrm{N} / \mathrm{I}$ and $\mathrm{I} / \mathrm{E}$ boundaries for experience (set at 0.5 and 100 , respectively), the horizontal dashed lines representing the corresponding cutoff-values for time task 3.

Traditionally, surgical competence has been based on the number of procedures performed, but there is no consensus on the exact number of performances of specific procedures required to distinguish between who is truly competent and who is not. ${ }^{19-21}$

We tested the arbitrariness of our definition of the expert level by comparing the pass rates of the residents in this study achieved with this level and with the expert level set at $>50$ and $>200$ laparoscopic procedures.

\section{Statistical analysis}

Classical approaches to estimate reliability are to measure inter-, intra-examiner and test-re-test reliability. However, weaknesses of these approaches are that new data must be generated to test each source of error. Second when an error is identified, it is not compared with other sources of error and to what extent it affects the results when both errors interact. For example, reliability estimated by the relation between performer and different examiners (inter-rater reliability) does not address reliability estimated by interaction between performer and exercise.

Generalizability theory is an extension of the classical approach and it comprises a regression technique that models and quantifies relationships between variables to make predictions about reliability. 22,23 The prediction is not restricted to real performances, but the model estimates reliability changes related to changes in variables, e.g. number of examiners or number of trials. In this study the generalizability analysis includes all variance components, i.e. 'performer' and 'rater' for the quality measure and 'performer' 
and 'trial' for the time measurement, by mathematical modeling. ${ }^{23}$ The outcome parameter of the generalizability analysis is the generalizability coefficient on a scale of 0 to 1.0 , were 0 is lowest reliability and 1.0 is perfect reliability. A coefficient of at least 0.80 was considered acceptable. ${ }^{12,24}$

Linear regression analysis of the transformed versions log (time) and log (experience), and quality and log (experience) was performed for each task to establish cut-off scores for time and quality on the boundaries between novice/intermediate and intermediate/expert. The data were analyzed using the Statistical Package for the Social Sciences (SPSS) version 19. A p-level of $<0.05$ was considered to indicate statistical significance.

\section{RESULTS}

Included in the study were 13 medical students, 20 second-year urology residents, and 17 urologists, together comprising 15 novices, 23 intermediates, and twelve experts. Novices, intermediates and experts had mean ages of 27 years (SD; 9), 35 years (SD; 5), and 46 years (SD; 5), respectively and they had performed a mean number of procedures of 0, 46 (SD: 35), and 658 (SD: 490), respectively. We excluded one participant from the analysis of task five and two participants from the analysis of task four, all from the intermediate group, because they had performed only part of the protocol due to time constraints during the course.

\section{Reliability of the PLUS-assessment}

The reliability, indicated by the generalizability coefficient (GC), was $0.95,0.81,0.88$, 0.81 , and 0.8 , respectively (Table I). Each additional trial predicted by the generalizability model increases the reliability of the assessment. With two examiners the inter-rater reli-

Table 3. PLUS-assessment cut-off scores for time per task and for quality of performance at the boundaries of novice/intermediate and intermediate/expert performances

\begin{tabular}{ccc} 
& Novice / Intermediate & Intermediate / Expert * \\
\cline { 2 - 3 } & Cut-off scores. & Cut-off scores. \\
"Time in seconds per task" and "quality" & "Time in seconds per task" and "quality" \\
Task 1 & 112 seconds & 86 seconds \\
Task 2 & 118 seconds & 91 seconds \\
Task 3 & 283 seconds & 149 seconds \\
Task 4 & 251 seconds & 188 seconds \\
Quality & 218 seconds & 163 seconds \\
\hline
\end{tabular}

* Expert level was defined as performance of $>100$ laparoscopic procedures 
ability showed a GC of 0.79 , increasing to $0.85 ; 0.88$, and 0.90 with three, four, and five examiners, respectively (Table II).

\section{Setting a pass/fail score for second-year residents}

The second-year residents had performed between nine and 100 laparoscopic procedures. Table III shows the cut-off scores for time and quality of performance per task on the novice/intermediate and intermediate/expert boundaries, with expert level set at $>100$ laparoscopic procedures. The pass percentages of the second-year residents were $63.64 \%$ for the novice/intermediate boundary and $9.09 \%$ for the intermediate/expert boundary. With expert level set at $>50$ procedures, the pass rate on the intermediate/ expert boundary remained $9.09 \%$, while an increase to $>200$ procedures reduced it to $6.06 \%$ (figure 3 ).

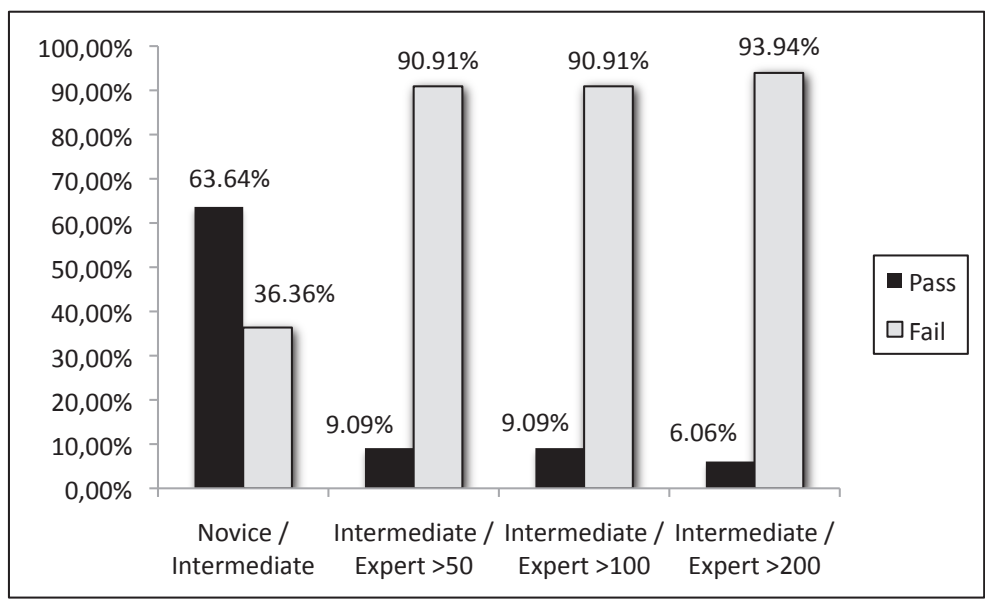

Figure 3. Pass rates of second-year residents for the PLUS assessment. Pass rates are shown for different cut-off values at the boundaries of novice/intermediate and intermediate/expert performances. The Y-axis indicates pass rates with the expert level set at $>50,>100$ and $>200$ laparoscopic procedures. Pass rates were measured over the mean score of two trials.

\section{DISCUSSION}

In this study of the PLUS assessment, we estimated the reliability of the assessment and determined a tenable minimal proficiency standard for second-year urological residents. The results of the generalizability analysis suggest that the PLUS is a reliable assessment tool for credentialing purposes, with two judges and two trials being sufficient to reach good reliability, although inter-rater reliability was slightly $<0.8$. These samples are feasible in terms of effort and resources. 
The most challenging, but crucial aspect of assessment is setting a pass/fail score that will filter out the truly incompetent examinees. Based on the results, we recommend setting the pass/fail standard for second-year urological residents for 'time' and 'quality' of performance of the five PLUS tasks at the boundary between novice and intermediate level. We believe that such a standard is tenable as a starting point for residents' further competency development towards the intermediate and expert levels during the next four years of their residency. Future research should focus on assessment methods and proficiency standards for experienced laparoscopists, since training and maintenance of skills is continuous throughout the career of surgeons.

Our methodology may seem somewhat unconventional compared to that of other studies examining performance standards for surgical skills, which generally set pass/ fail scores based on novice versus expert performances or sometimes on expert performance only. ${ }^{25,26}$ It is generally advocated, however, that pass/fail scores for a certain group of examinees should be based on the expected performance of that specific group. ${ }^{5,18,27,28}$ We therefore deem the generalized examinee-centered method to be optimal for setting a pass/fail score for the PLUS assessment of second-year residents, as it is consistent with the principle of continuous progress of performance from novice to expert. ${ }^{18,29}$

The PLUS assessment requires performance of all tasks within the pass/fail scores for time and quality. Although this may seem a very stringent criterion, because it does not allow for any compensation between the outcomes for different tasks, we think it is tenable, because the tasks are of increasing level of difficulty and assess different aspects of basic laparoscopic skills.

A limitation of studies aimed at setting performance standards is the inevitability of arbitrariness, especially when the pass/fail score is based on a definition of expert performance. ${ }^{21}$ Using the total number of procedures performed as a surrogate measure of clinical competence may be problematic, because an accurate definition depends on how an acceptable outcome is defined. In this study the definition of the expert level was based on studies showing a significant reduction in complications of urological laparoscopy after $>100$ procedures..$^{19,20}$ Our results showed no evidence of arbitrariness with a definition of the expert level based on 50 or 200 procedures. This suggests that 50 performed procedures appear to be an acceptable level of expert performance for basic laparoscopic skills, highlighting the necessity of further skills development during a stepwise residency curriculum.

A study to determine whether basic skills performance is transferable to performance in patients would in all likelihood be considered unethical. Nevertheless, it is important to aim at a standard with the lowest false-positive and false-negative results. Be that as it may, such a standard is likely out of reach for basic laparoscopic skills, for, while the accuracy of diagnostic tests in establishing the presence of a certain disease can be 
proven by pathological or bacteriological analysis, the accuracy of basic laparoscopic skills performance by residents who are still in the learning curve is impossible to underpin by actual surgical outcomes.

We therefore recommend to measure criterion validation of the PLUS assessment on animal models in future studies and developing a stepwise training program, integrating technical skill training, cognitive processing, attitude and knowledge. ${ }^{9,30,31}$ Future studies should focus on the question to what extend different simulators, such as low-fidelity simulators, virtual reality simulators, animal models and supervised patient care can complement each other to develop a comprehensive training and assessment program for basic and procedural skills. ${ }^{3,10,32-34}$ Setting tenable performance standards, however, is an important matter regardless of any kind of simulation training to improve its assessment purposes.

It should be noted that a pass score on the PLUS assessment cannot be interpreted as proof of competence in procedural skills, but only as proof of second-year residents meeting the very minimum standard of basic technical proficiency as a starting point for their further training trajectory towards the next level of the laparoscopic curriculum.

\section{CONCLUSION}

In this study, we aimed to promote the standardization of assessment during urological residency training. We did so by demonstrating the reliability of the PLUS assessment and by establishing a pass/fail score for basic skills performance of second-year urological residents. It appears that the pass/fail score on the novice/intermediate boundary offers a tenable starting point from which residents can be allowed to continue their development towards the next level in the laparoscopic curriculum. We recommend integrating assessments at several stages of the laparoscopic curriculum to ensure uniformity and standardization of skills competency.

\section{Acknowledgment}

The authors would like to thank all the medicals students, residents in urology and urologists, who participated in the development of the PLUS training and testing. The authors would also like to thank Mereke Gorsira for her editorial assistance. 


\section{REFERENCES}

1. Imkamp F, Herrmann TR, Rassweiler J, et al. Laparoscopy in German urology: changing acceptance among urologists. Eur Urol. 2009; 56: 1074-80.

2. Parsons BA, Blencowe NS, Hollowood AD and Grant JR. Surgical training: the impact of changes in curriculum and experience. J Surg Educ. 2011; 68: 44-51.

3. Schijven and Jakimowicz. Simulators, first experiences. Minim Invasive Ther Allied Technol. 2003; 12: 151-4.

4. Kelly BD, Curtin PD and Corcoran M. The effects of the European Working Time Directive on surgical training: the basic surgical trainee's perspective. Ir J Med Sci. 2011; 180: 435-7.

5. Kroeze SG, Mayer EK, Chopra S, Aggarwal R, Darzi A and Patel A. Assessment of laparoscopic suturing skills of urology residents: a pan-European study. Eur Urol. 2009; 56: 865-72.

6. McDougall EM. Validation of surgical simulators. J Endourol. 2007; 21:244-7.

7. Shaw K, Cassel CK, Black C and Levinson W. Shared medical regulation in a time of increasing calls for accountability and transparency: comparison of recertification in the United States, Canada, and the United Kingdom. JAMA. 2009; 302: 2008-14.

8. Stassen LP, Bemelman WA and Meijerink J. Risks of minimally invasive surgery underestimated: a report of the Dutch Health Care Inspectorate. Surg Endosc. 2010; 24: 495-8.

9. Hasson HM. Core competency in laparoendoscopic surgery. JSLS : Journal of the Society of Laparoendoscopic Surgeons / Society of Laparoendoscopic Surgeons. 2006; 10: 16-20.

10. Laguna MP, Arce-Alcazar A, Mochtar CA, Van Velthoven R, Peltier A and de la Rosette JJ. Construct validity of the chicken model in the simulation of laparoscopic radical prostatectomy suture. $J$ Endourol. 2006; 20: 69-73.

11. Searle J. Defining competency - the role of standard setting. Med Educ. 2000; 34: 363-6.

12. Wass VA, J. Assessing Learners. In: Dornan TM, K.; Scherpbier, A.; Spencer, J. , (ed.). Medical Education Theory and Practice. London: Churchill Livingstone Elsevier, 2011, p. 229-56.

13. Norcini JS, J.A. The Credibility and Comparability of Standards. Appl Meas Educ. 1997; 10: 39-59.

14. Norcini JJ. Setting standards on educational tests. Med Educ. 2003; 37: 464-9.

15. Vleuten CPMC-S, J. Standard Setting In: Patil NC, L.K.; , (ed.). Assessment in Medical and Health Sciences Education. Hong Kong: Institute of Medical and Health Sciences Education, 2009.

16. Moorthy K, Munz Y, Dosis A, Bello F, Chang A and Darzi A. Bimodal assessment of laparoscopic suturing skills: construct and concurrent validity. Surg Endosc. 2004; 18: 1608-12.

17. Aggarwal $R$, Hance J, Undre $S$, et al. Training junior operative residents in laparoscopic suturing skills is feasible and efficacious. Surgery. 2006; 139: 729-34.

18. Cohen ASK, M.T.; Crooks T.J. A generalized examinee-centered method for setting standards on achievement tests. Appl Meas Educ. 1999; 12: 343-66.

19. Fahlenkamp D, Rassweiler J, Fornara P, Frede T and Loening SA. Complications of laparoscopic procedures in urology: experience with 2,407 procedures at 4 German centers. J Urol. 1999; 162: 765-70; discussion 70-1.

20. Soulie M, Seguin P, Richeux L, et al. Urological complications of laparoscopic surgery: experience with 350 procedures at a single center. J Urol. 2001; 165: 1960-3.

21. Van Nortwick SS, Lendvay TS, Jensen AR, Wright AS, Horvath KD and Kim S. Methodologies for establishing validity in surgical simulation studies. Surgery. 2010; 147: 622-30.

22. Crossley J, Russell J, Jolly B, et al. 'I'm pickin' up good regressions': the governance of generalisability analyses. Med Educ. 2007; 41: 926-34. 
23. Crossley J, Davies H, Humphris G and Jolly B. Generalisability: a key to unlock professional assessment. Med Educ. 2002; 36: 972-8.

24. Downing SM. Reliability: on the reproducibility of assessment data. Med Educ. 2004; 38: 1006-12.

25. Fraser SA, Klassen DR, Feldman LS, Ghitulescu GA, Stanbridge D and Fried GM. Evaluating laparoscopic skills: setting the pass/fail score for the MISTELS system. Surg Endosc. 2003; 17: 964-7.

26. Verdaasdonk EG, Dankelman J, Lange JF and Stassen LP. Incorporation of proficiency criteria for basic laparoscopic skills training: how does it work? Surg Endosc. 2008; 22: 2609-15.

27. Korndorffer JR, Jr., Stefanidis D and Scott DJ. Laparoscopic skills laboratories: current assessment and a call for resident training standards. Am J Surg. 2006; 191: 17-22.

28. Wass V, Van der Vleuten C, Shatzer J and Jones R. Assessment of clinical competence. Lancet. 2001; 357: 945-9.

29. Kneebone RN, D. Learning and teaching clinical procedures,. In: Dornan TM, K.; Scherpbier, A.; Spencer, J., (ed.). Medical Education: Theory and Practice. London: Churchill Livingstone Elsevier, 2011, p. 171-91.

30. Pugh CM, DaRosa DA, Santacaterina S and Clark RE. Faculty evaluation of simulation-based modules for assessment of intraoperative decision making. Surgery. 2011; 149: 534-42.

31. Schijven MP and Bemelman WA. Problems and pitfalls in modern competency-based laparoscopic training. Surg Endosc. 2011; 25: 2159-63.

32. Verdaasdonk EG, Dankelman J, Schijven MP, Lange JF, Wentink M and Stassen LP. Serious gaming and voluntary laparoscopic skills training: a multicenter study. Minim Invasive Ther Allied Technol. 2009; 18: 232-8.

33. Schijven MP and Jakimowicz JJ. Validation of virtual reality simulators: Key to the successful integration of a novel teaching technology into minimal access surgery. Minim Invasive Ther Allied Technol. 2005; 14: 244-6.

34. Autorino R, Haber GP, Stein RJ, et al. Laparoscopic training in urology: critical analysis of current evidence. J Endourol. 2010; 24: 1377-90. 




\section{ABSTRACT}

\section{Background}

In 2011, the European Basic Laparoscopic Urological Skills (E-BLUS) examination was introduced as a pilot for the examination of final-year urological residents.

\section{Objective}

In this study we aimed to answer the following research questions: What level of laparoscopic skills do final-year residents in urology have in Europe, and do the participants of the E-BLUS pass the examination according to the validated criteria?

\section{Design, setting and participants}

Participants of the examination were final-year urology residents from different European countries taking part in the European Urology Residents Education Program in 2011 and 2012.

\section{Surgical Procedure}

The E-BLUS exam consists of 5 tasks validated for the training of basic urologic laparoscopic skills.

\section{Outcome measurements and statistical analysis}

Performances of the tasks were recorded on DVD and analysed by an objective rater. Time and number of errors made in tasks 1-4 were noted. Furthermore, all expert laparoscopic urologists were asked to score participants on a global rating scale (1-5) based on three items: depth perception, bimanual dexterity and efficiency. Participants were asked to complete a questionnaire on prior training and laparoscopic experience.

\section{Results and limitations}

Seventy DVD recordings were analysed. Most participants did not pass time criteria on task 4 (90\%), task 2 (85.7\%), task 1 (74.3\%) and task 5 (71.4\%). Task 3 was passed by $84.3 \%$. The overall quality score was passed by $64 \%$. When combining time and quality, only 3 participants (4.2\%) passed the examination according to the validated criteria. According to the questionnaire, $61 \%$ did not have the opportunity to train laparoscopic skills.

\section{Conclusions}

The results of the E-BLUS examination show that the level of basic laparoscopic skills among European residents is low. Although quality of performance is good, most residents do not pass the validated time criteria. Regular laparoscopic training or a dedicated fellowship in laparoscopy should improve the laparoscopic level of residents in urology. 


\section{INTRODUCTION}

Despite the growing popularity of robot-assisted laparoscopy in urology, conventional laparoscopic surgery is still the established technique for several indications throughout Europe.' Many of the laparoscopic procedures have a lengthy learning curve, because laparoscopy requires other skills than open surgery and robot-assisted laparoscopy, such as counter intuitive movements of the instruments and an indirect view of the operating site. ${ }^{2,3}$ There is a recognized need of a more formalised laparoscopic training framework within urology to overcome the difficulties of this technique and to shift the first part of the learning curve from the patient to the skills laboratory. Therefore, simulator based skills training has been widely accepted and implemented. ${ }^{4}$

However, the qualification and certification of laparoscopic skills performance are still in a preliminary phase within urology. In response to urgent calls from the government and the public for well-defined proficiency standards to safeguard the quality of care, we developed the program for laparoscopic urological skills (PLUS). ${ }^{5,6}$ The PLUS has been validated by a cohort of laparoscopic experts, intermediates and novices in the Netherlands, and its face, content, and construct validity has been proven. The PLUS examination offers quality criteria and time criteria for the completion of basic laparoscopic tasks and a certification standard for residents based on the generalized examinee-centred method. The pass/fail criteria for time and quality of performance per task were set on the novice/intermediate boundaries. ${ }^{6}$ In the Netherlands, PLUS has recently been implemented at a national level as a "basic laparoscopy examination".

In 2011, the PLUS was introduced at the European level as a pilot for the examination of final-year urologic residents. It is called the European Basic Laparoscopic Urological Skills (E-BLUS) examination.

In this study, we aimed to answer the following research questions: What level of laparoscopic skills do final-year residents in urology in Europe have, and do participants of the E-BLUS pass the examination according to the previously validated criteria?

\section{METHOD}

\section{Setting}

The E-BLUS examination was conducted during the laparoscopic hands-on training (HOT) section of the European Urological Residents Education Programme (EUREP) meetings 2011 and 2012. EUREP is organized annually by the European School of Urology (ESU) in collaboration with the European Board of Urology and has been developed exclusively for European residents. Participation in the E-BLUS examination was facilitated by prior online registration on a voluntary basis. All participants were advised to attend an HOT 
session prior to the examination in which they could familiarise themselves with the exercises.

\section{Materials}

The E-BLUS examination consists of 5 tasks (Fig. 1). With these tasks, the examination assesses bimanual dexterity, hand-eye coordination, spatial awareness, suture technique, and clipping and cutting skills. All participants in the study used identical boxes, tasks, suture material (Polysorb 3-0, Tyco Healthcare, Mansfield, MA, USA), Hem-o-Lok appliers (Teleflex Medical, Research Triangle Park, Durham, NC, USA) and laparoscopic instruments (Olympus, Hamburg, Germany). The five tasks shown in Figure 1 have been previously described in the validation study of Tjiam et al. ${ }^{5,6}$, who established face, content and construct validity and determined test criteria.

Time and quality were measured for the tasks. To judge the quality, we used a binominal 14-item checklist covering the quality parameters. For each error, a score of 0 was applied. Target overall quality score was 11 out of 14 (Table 1).

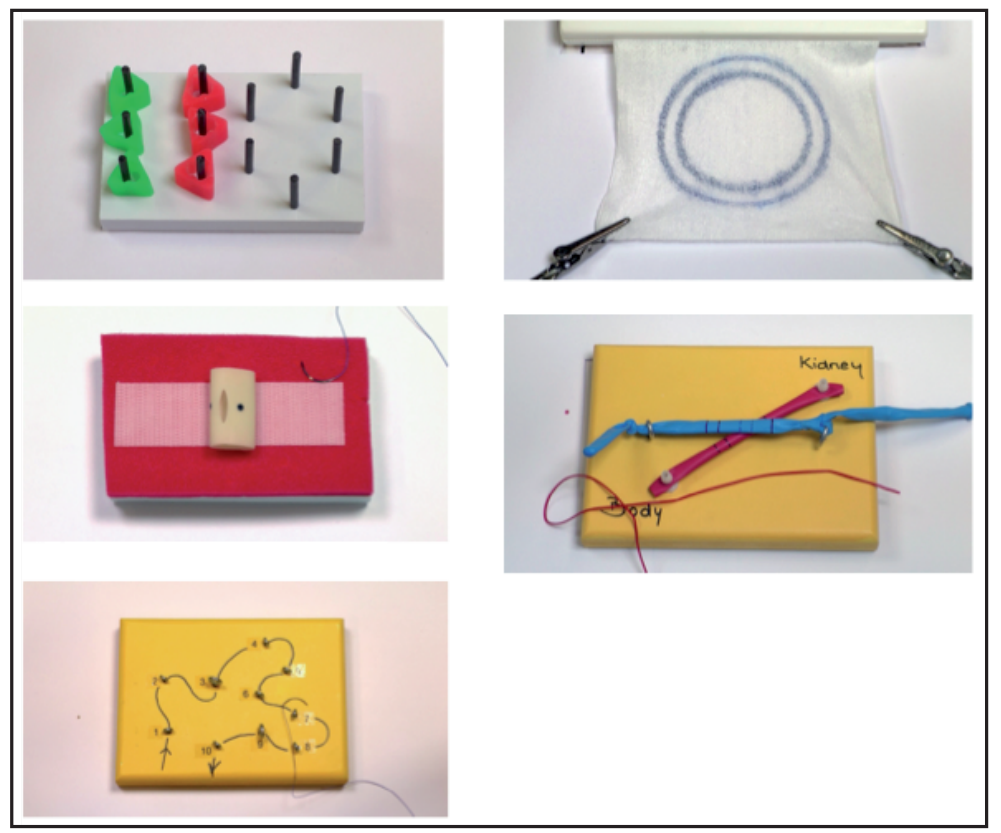

Figure 1. The E-BLUS tasks: The examination comprises five basic laparoscopic tasks in a box trainer with a fixed camera position 
Table 1. Binominal checklist used for the evaluation of quality

\begin{tabular}{|c|c|c|c|}
\hline & & Trial 1 & Trial 2 \\
\hline Task 1 & Number of dropped objects* & & \\
\hline Task 2 & $\begin{array}{l}\text { Cut between the lines? } \\
\text { (yes or no) }\end{array}$ & & \\
\hline \multirow[t]{3}{*}{ Task 3} & $\begin{array}{l}\text { Suture is placed within } 1 \mathrm{~mm} \text { or through the dots? } \\
\text { (yes or no) }\end{array}$ & & \\
\hline & $\begin{array}{l}\text { The knot holds (does not slip)? } \\
\text { (yes or no) }\end{array}$ & & \\
\hline & $\begin{array}{l}\text { The knot keeps approximation of the tissue? } \\
\text { (yes or no) }\end{array}$ & & \\
\hline \multirow[t]{3}{*}{ Task 4} & $\begin{array}{l}\text { Three clips are place on both tubes? } \\
\text { (yes or no) }\end{array}$ & & \\
\hline & $\begin{array}{l}\text { All clips are placed within } 1 \mathrm{~mm} \text { of the line? } \\
\text { (yes or no) }\end{array}$ & & \\
\hline & $\begin{array}{l}\text { All cuts are placed between the dotted lines? } \\
\text { (yes or no) }\end{array}$ & & \\
\hline
\end{tabular}

- Number of objects was counted but equal to the validation study of Tjiam et al. ${ }^{5,6}$ not included in the overall quality score

In summary, the following task descriptions, error criteria and target scores were used:

- Task 1: Peg transfer. Six plastic objects are grasped, transferred to the opposite forceps, placed on a pegboard, and visa versa. The number of dropped objects was counted. The target time was: $112 \mathrm{~s}$. This task required two dissectors.

- Task 2: Pattern cutting. A circle is cut from gauze between two premarked lines. A cut beyond the outer or inner line of the circle is scored as an error. The target time: $118 \mathrm{~s}$. This task required a dissector and a pair of scissors.

- Task 3: Single knot tying. An intracorporeal knot is made on a Penrose drain. A stitch beyond $1 \mathrm{~mm}$ of the black dots, a gap in the slit of the Penrose drain or a slipping knot was scored as an error. The target time was 283s. This task required two needle holders.

- Task 4: Clip and cut. Hem-o-Lok clips are placed around two tubes, and the tubes are cut. One of the clips placed outside of $1 \mathrm{~mm}$ of the continuous line or a cut beyond the dotted lines was scored as an error. The target time was $251 \mathrm{~s}$. This task required two dissectors, a pair of scissors and a Hem-o-Lok applier.

- Task 5: Needle guidance. A needle is guided through 10 metal rings following a set route. The target time was $218 \mathrm{~s}$. This task required two needle holders.

Participants were allowed to practise each task for 1 minute prior to the examination and had to perform the exercise twice during the examination. 


\section{Instruments}

The pass/fail standard of the examination based on time and quality of performance was derived from the publication of Tjiam et al. ${ }^{6}$ and based on the generalized examineecentred method described by Cohen et al.. ${ }^{7}$ This educational approach uses the linear relationship between assessment scores and degree of procedural experience of multiple reference groups. The pass/fail scores were set as described by Tjiam et al. - that is, on the boundaries between the categories of novices (0 laparoscopic procedures performed) and intermediate experience in laparoscopy (between 1 and 100 procedures performed) - as a starting point for residents' further competency development towards the intermediate and expert levels.

Before the start of the examination, each participant was instructed by an expert laparoscopic urologist who had attended a teach-the-teacher course. The teach-the-teacher course focused on the background of the examination, the criteria, and the explanation the examinees were to receive during the examination. Performance was measured by recording time with a stopwatch and registering the number of errors made in tasks $1-4$. Furthermore, all expert laparoscopic urologists were asked to score participants on a global rating scale $(1-5)$ based on three items: depth perception, bimanual dexterity and efficiency.

To minimise the effect of interrater differences, each task was recorded by digital video and saved for rating by independent raters. Ten DVD recordings were rated by two researchers to check whether the rater reliability were sufficiently high to allow a single rater. Classical approaches estimate reliability by measuring inter- and intraexaminer reliability, but weaknesses of these approaches are that new data must be generated to test each source of error. Moreover, when an error is identified, it is not compared with other sources of error, nor do these approaches assess to what extent the results are affected when errors interact. For example, reliability estimated by the relation between performer and other examiners (inter rater reliability) does not address reliability estimated by interaction between performer and exercise. To avoid these weaknesses, we applied the generalizability theory. This theory comprises a regression technique that models and quantifies relationships between variables to make predictions about reliability. In current study, the generalizability analysis included the variance components for performer, examiner, and the interaction performer $x$ examiner. The generalizability coefficient was measured on a scale of 0 to 1.0 , where 0 was lowest reliability and 1.0 was perfect reliability ${ }^{8}$ The generalizability coefficient for two video observers for time was near perfect $(G>0.99)$ for all five tasks. Therefore, the data of a single rater were used for further analysis. 


\section{Questionnaire}

At the end of the examination, each resident was asked to complete a questionnaire (Fig.2). The questionnaire consisted of three sections. The first section concerned demographics and postgraduate year of training, and the second section covered experience in actual laparoscopic procedures. In the third section, the residents were asked how many hours they had trained in laparoscopic skills in the $4 \mathrm{wk}$ prior to the examination, whether there was a skills laboratory in their hospital, whether laparoscopic simulation devices were available, and whether they had ever attended a laparoscopic HOT course before the EUREP.

\section{Outcome measures}

The primary end point was to compare the results of the examination with the validated criteria. The secondary end point was to determine whether a relationship existed between laparoscopic skills and the participants' previous laparoscopic experience and training.

\section{Statistical analysis}

Statistical analysis was performed using SPSS version 18 software (IBM Copr., Armonk, NY, USA). Regression analysis used exam results as dependent variables and the variables in the questionnaire as independent variables. The statistical significance of a regression coefficient was tested by a t test, considering a result statistically significant if $p<0.05$.

\section{RESULTS}

In total, 104 participants from 20 different countries completed the exam in 2011 and 2012. We analysed all the available DVD recordings of their performances $(n=70)$. Because no video recordings of the other 34 participants were available, we excluded them from analysis. The mean age of the participants was $31 \mathrm{yr}$ of age (range: $26-40$ ), and mean year of residency was 5 (range: 2-6).

Of the included participants, the majority did not pass the time criteria on task $4(90.0 \%$ failed), task 2 (85.7\% failed), task 1 (74.3\% failed) and task 5 (71.4\% failed). Participants scored better on task 3 , the intra-corporeal suturing exercise, than the other exercises. This exercise was passed in time by $84.3 \%$ of the participants. Forty-five of 70 participants (64.3\%) (Fig. 3) passed the quality criteria. Only three participants (4.2\%) passed the combination of time and quality criteria. According to the questionnaire results, $65 \%$ of the participants did not have a skills lab in their hospital, and $61 \%$ did not have the opportunity to train laparoscopic skills. Also, $61 \%$ declared that they had not train in basic laparoscopic skills in the four weeks prior to the examination. The European final- 


\section{Questionnaire BLUS-assessment EUREP 2011}

-Your responses will be processed anonymously-

Number:

Age:.

Country:

Year of residency:

Describe your residency program: (Example: in the Netherlands the urology curriculum is 6 years in total: 2 years general surgery and 4 years urology)

\section{Questions considering laparoscopic experience}

How many laparoscopic operations did you perform independent with our without supervision?

(Please write down the number per procedures below)

- Lap. Cholecystectomy:

- Lap. Appendectomy:

- Lap. Hernia Inguinalis repair:

- Lap. Hemicolectomy:

- Lap. Nephrectomy:

- Lap. Partial Nephrectomy:

- Lap. Pyeleoplasty:

- Lap. Lymph Node Dissection:

- Lap. Orchidectomy:

- Lap. Prostatectomy:

- Lap. Cystectomy:

- Lap. Adrenalectomy:

- Other: 


\section{Questions considering training facilities}

1. Does your hospital have a skills laboratory?

Yes/ No

2. Does your hospital provide facilities for training laparoscopic skills?

Yes/No

(If "No", please continue with question 6)

3. What kind of simulators does your hospital provide?

$\square$ Box-trainer

$\square$ VR-simulator

$\square$ Other

Figure 2. The questionnaire on prior training and laparoscopic experience

year urology residents performed a median of four laparoscopic procedures during their residency, including previous general surgery laparoscopic experience.

Global rating scores scored by the expert laparoscopic urologists on depth perception were on average 3.6 (range: 1-5), 3.7 on dexterity (range: $2-5$ ) and 3.5 on efficiency (range: $1-5$ ). For participants who had previously attended HOT courses, the global rating score was found to be significantly higher (difference: 0.3 ; $t$ test, $p<0.05$ ). A participant's previous training and previous experience did not affect the time scores, nor the quality scores obtained at the examination. 

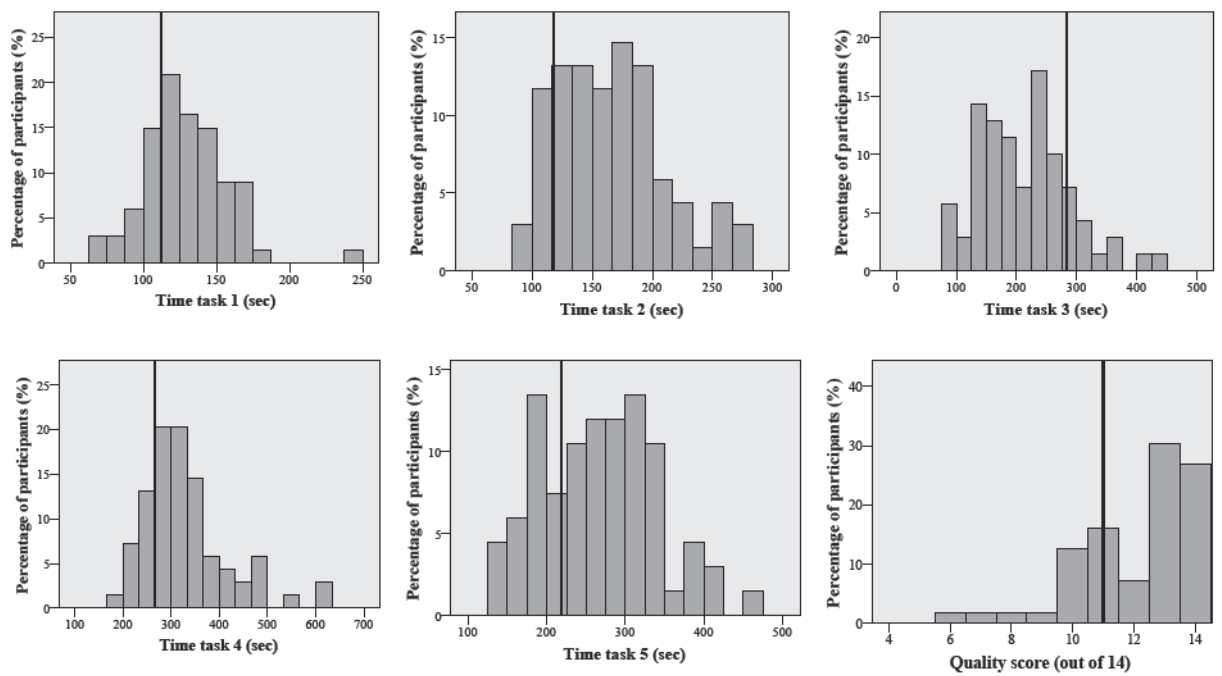

- cut-off time score according to the validated criteria

Figure 3. Graphs of all participants' scores on separate tasks and the overall quality score compared with validated criteria.

\section{DISCUSSION}

The first results of the E-BLUS examination showed that the majority of participants did not meet the E-BLUS criteria. Few final-year residents passed the examination according to the validated criteria. Results of the questionnaire revealed that overall training experience was limited and that most participants had not trained prior to the examination. Also, final-year residents in urology appeared to have limited exposure to actual laparoscopic procedures.

The scores may be relatively low for different reasons. One reason could be that the criteria set in previous research by Tjiam et al. ${ }^{6}$ were too strict, but this does not seem likely. We know from literature on the Fundamentals of Laparoscopic Surgery examination that most novice participants can reach even strict criteria based on expert scores and that it is a matter of training effort ${ }^{9}$. This is not surprising and can be explained by Ericsson's theory that deliberate practice is the most important ingredient for developing expertise. ${ }^{10}$ The PLUS criteria are set on the boundaries of novices' and intermediates' performances and were intended to be not extremely difficult.

Another reason could be that participants may not have been capable or not sufficiently prepared. We think that the results of the questionnaire explain why participants failed to reach the criteria. The low level of experience in laparoscopic procedures of the final-year residents had not been compensated by regular basic skills training. Most of 
them had not trained at all in the 4 wk prior to the exam or had not even been able to practice basic skills in their hospital.

The suturing task was performed relatively well within time. This is an interesting finding, because laparoscopic suturing is supposed to be one of the most challenging laparoscopic skills. Previous research on the EUREP meeting confirmed the appropriate level of suturing, especially among more experienced residents. ${ }^{11}$ This finding can be explained by the fact that the residents were generally able to perform the laparoscopic tasks, which indicates that they were not clumsy or incompetent, but because they were not sufficiently trained or experienced, performing the tasks within the time limit was the main issue. The time criteria for the suturing task were not set so strictly, because the criteria of the PLUS were based on a generalized examinee-centered method based on the boundaries between novices and intermediates, and suturing is considered a challenging skill even for laparoscopists with intermediate experience. Therefore, the criteria of the suturing task seem to be strict on quality rather than on time. This may explain why residents scored relatively better on this task. The overall quality score, which was good for most participants, confirms that they scored better on quality of performance than on time for all tasks. This is also in line with the global rating scores received by the expert laparoscopic urologists.The question these results raise is whether it is really necessary to speed up the basic laparoscopic skills and whether a good performance on quality is not more important in a clinical setting. The phases of learning a new motor skill have been described previously. ${ }^{12}$ The first phase is on quality and accuracy, while speed and time are emphasized in a second phase, and, finally, time-sharing is introduced to obtain full automaticity of the skill. The participants of the E-BLUS examination performed relatively well on accuracy and quality, but they need more practice to increase their speed before they reach automaticity in these skills. In our opinion, not passing the time criteria means that these participants were not close to automaticity, yet. By acquiring basic skills and training these skills to automaticity in a preclinical setting, residents can concentrate on the performance of the actual procedure and on all the procedural steps in the operating room. By intensifying basic laparoscopic skills training, a larger part of the learning curve of laparoscopic surgery can be shifted from the patient to the skills laboratory. The training in the operating room can subsequently be used for time-sharing tasks, such as dealing with procedural steps, difficult anatomy or complications, while less attention is needed for the technical difficulties of laparoscopic surgery, such as counter-intuitive movements.

Residents who are willing to perform laparoscopic surgery should train in laparoscopic skills on a regular basis. This is in line with previous research by Stolzenburg et al. ${ }^{13}$, who suggest that those who are willing to learn the laparoscopic prostatectomy should practice daily on a pelvic trainer, especially knot tying and suturing. Kroeze et al. ${ }^{11}$ stated that modular simulator training as part of a formal training programme may help to 
overcome some of the shortfalls in residents' exposure to laparoscopic procedures as a primary surgeon. Laguna et al. ${ }^{14}$ stated that it is almost impossible to finalise the residency training as a qualified laparoscopic surgeon. Based on the low level of laparoscopic skills of last year's residents, it is advisable that those who are willing to perform laparoscopy as a urologist should improve training and exposure to laparoscopy during residency or should consider a post-residency fellowship in laparoscopy.

A limitation of this study is that we could not define the parameters that predict passing a score on the E-BLUS examination. Neither previous experience nor in the questionnaire-administered previous training experience significantly correlated with the overall score on the examination. Possibly, the participants' self-reported experience was not an accurate indication of their actual experience; alternatively, the international and intercultural differences in residency training may have been too large to compare operative and training experience. Another limitation is that not all final-year residents attend EUREP and that only a portion of them partakes in the E-BLUS examination. Possibly, this may have caused a selection bias, resulting in a different level of laparoscopic skills among the participants of the exam compared to the general population of finalyear residents.

\section{CONCLUSION}

The first results of the E-BLUS examination show that the level of basic laparoscopic skills among European residents is low. Although the quality of performance is good, most residents do not pass the validated time criteria of the E-BLUS examination. The timing and setting of the examination should be carefully evaluated to determine its future use. Moreover, regular laparoscopic training or a dedicated fellowship in laparoscopy should improve the laparoscopic level of residents in urology who intend to perform laparoscopy.

\section{Acknowledgement}

The authors acknowledge the ESU and EUREP organisers for allowing them to conduct this study during EUREP, and they thank the training faculty for their assistance in the collection of the data. The authors would also like to thank Malou Kuppen for her help in reviewing the DVD recordings, Jeroen van Merriënboer for his critical review of this manuscript, and Lisette van Hulst for her editorial assistance. 


\section{Funding/Support and Role of the Sponsor}

Olympus kindly supported the project with the loan of workstations, laparoscopic equipment and recording equipment and with logistics, and an unrestricted educational grant to the ESU for support of the EUREP. 


\section{REFERENCES}

1. McDougall EM and Clayman RV. Advances in laparoscopic urology part I. History and development of procedures. Urology. 1994; 43: 420-6.

2. Rassweiler J, Sentker L, Seemann O, Hatzinger M, Stock C and Frede T. Heilbronn Laparoscopic radical prostatectomy. European urology. 2001; 40: 54-64.

3. Poulakis V, Dillenburg W, Moeckel M, et al. Laparoscopic radical prostatectomy: prospective evaluation of the learning curve. European urology. 2005; 47: 167.

4. Parsons BA, Blencowe NS, Hollowood AD and Grant JR. Surgical training: the impact of changes in curriculum and experience. Journal of Surgical Education. 2011; 68: 44-51.

5. Tjiam IM, Persoon MC, Hendrikx AJ, Muijtjens AM, Witjes JA and Scherpbier AJ. Program for Laparoscopic Urologic Skills: A Newly Developed and Validated Educational Program. Urology. 2012; 79: 815-20.

6. Tjiam IM, Schout BM, Hendrikx AJ, et al. Program for laparoscopic urological skills assessment: Setting certification standards for residents. Minimally Invasive Therapy \& Allied Technologies. 2012: 1-7.

7. Cohen AS, Kane MT and Crooks TJ. A generalized examinee-centered method for setting standards on achievement tests. Applied Measurement in Education. 1999; 12: 343-66.

8. Crossley J, Davies H, Humphris $\mathrm{G}$ and Jolly B. Generalisability: a key to unlock professional assessment. Medical education. 2002; 36: 972-8.

9. Scott DJ, Ritter EM, Tesfay ST, Pimentel EA, Nagji A and Fried GM. Certification pass rate of $100 \%$ for fundamentals of laparoscopic surgery skills after proficiency-based training. Surgical endoscopy. 2008; 22: 1887-93.

10. Ericsson KA. Deliberate practice and the acquisition and maintenance of expert performance in medicine and related domains. Academic Medicine. 2004; 79: S70-S81.

11. Kroeze SG, Mayer EK, Chopra S, Aggarwal R, Darzi A and Patel A. Assessment of laparoscopic suturing skills of urology residents: A Pan-European study. European urology. 2009; 56: 865-73.

12. van Merrienboer $J$ and Kirschner P. Ten steps to complex learning. A systematic approach to fourcomponent instructional design. New York/London: Routledge, 2007.

13. Stolzenburg J-U, Truss MC, Rabenalt R, et al. Training in laparoscopy. eau-ebu update series. 2007; 5: 53-62.

14. Laguna MP, Schreuders L, Rassweiler J, et al. Development of laparoscopic surgery and training facilities in Europe: results of a survey of the European Society of Uro-Technology (ESUT). European urology. 2005; 47: 346-51. 



\section{ABSTRACT}

\section{Purpose}

To evaluate the place of the TURPsim ${ }^{\text {TM }}$ (Simbionix/Virtamed, Beit Goal, Israel) within a urological residency training curriculum, including training needs analysis (TNA), and investigating its validity.

\section{Material and Methods}

TNA was conducted by an expert panel to identify procedural steps and pitfalls. Performance metrics of the simulator were compared with the TNA results. Participants were distributed according to their level of experience (completed TURP procedures); Novices $(0)$, intermediates $(1-50)$ and experts $(>50)$. They followed standardized instructions and then performed 2 complete TURP procedures on the TURPsim ${ }^{\text {TM }}$.

\section{Results}

10 out of 22 procedural steps (TNA) and 4 out of 11 pitfalls were covered by the TURP$\operatorname{sim}^{\mathrm{TM}}$. A total of 66 participants, 22 in each group, were included. Median general judgement (face and content) about the TURPsim ${ }^{\text {TM }}$ was rated 7.3. (Median: 7; range 3-9). Ninety-three percent of all participants qualified the TURPsim ${ }^{\mathrm{TM}}$ as an useful training model. Intermediates and experts had a significant faster resection time and less blood loss compared to novices (construct) $(p=0.001)$. Novices needed to re-resected previous lobes and they also resected the prostate in the incorrect order more frequently compared to intermediates and experts.

\section{Conclusion}

TNA is of paramount importance in the evaluation process of a training program. This curriculum based approach including validity of a simulator seems valuable and may narrow the gap between skillslab and clinical practice. This study showed face, content and construct validity of the TURPsim ${ }^{\mathrm{TM}}$ and this simulator founds its place in the current urological curriculum to train basic and procedural TURP skills. 


\section{INTRODUCTION}

Simulator based training is widely used in surgical skills education as an adjunct to learning in clinical practice. Key advantage is that it allows trainees to gain cognitive knowledge and procedural skills in a safe environment, where mistakes can be made and where the trainee can practice deliberately. ${ }^{1-3}$ It is generally acknowledged that "training needs analysis" (TNA) should be the first step in development of an educational intervention, including simulator based training. ${ }^{4-6}$ Training needs analysis is described as the process of identifying the gap in training and related training needs; "are trainees able to perform the procedure or is there a performance problem?" "What may be possible causes for this performance problem?" "Can the performance problem be solved with extra training outside the operation theatre?"?, 8 Training needs analysis is primarily conducted to determine where training is needed, what needs to be taught and who needs to be trained. One of the outcomes is the specification of training objectives, including identification of procedural steps and analysis of pitfalls when designing training for surgical skills.9. 10

Before implementing a simulator in training curricula it should be evaluated whether it trains what it is supposed to train, also known as its validity. According to the definitions of McDougal et al. validity has been described as usefulness and realism of the simulator according to the opinions of novices (face validity) and experts (content validity). Moreover, it should differentiate between different levels of expertise (construct validity), using adequate outcome parameters. ${ }^{11}$

Since decennia, transurethral resection of the prostate (TURP) has been the "golden standard" in surgical treatment for symptomatic benign prostatic hyperplasia (BPH). ${ }^{12}$ ${ }^{13}$ Literature describes that this procedure requires fifty to hundreds of cases before achieving expertise. ${ }^{14,15}$ However, recent increase of alternative treatments for benign prostatic hyperplasia, such as medical therapy and holmium laser enucleation of the prostate have decreased the number of procedures available for residents to learn the many complex tasks of the TURP. ${ }^{16}$ In addition, the former apprenticeship-training model is outdated and the "working time directive" restricts the amount of learning by doing during residency. ${ }^{17}$

In this study we investigated the main research question: "Does TURP virtual realitysimulator complement learning in practice in the urological residency curriculum?" To answer this research question, we divided our study into four sub-questions:

1) What are the steps in a TURP procedure and which steps could be trained on a simulator according to a TNA using expert and resident opinions? 2) To what degree does the TURP on the TURPsim ${ }^{\mathrm{TM}}$ resemble real life procedures according to the opinion of students and urology residents (face validity), and urologists (content validity)? 3) Is discrimination between different levels of expertise possible using the TURPsim ${ }^{\mathrm{TM}}$ 
(construct validity)? 4) How useful can the TURPsim ${ }^{\text {TM }}$ be for urology training according to urology residents and urologists?

\section{MATERIAL AND METHODS}

\section{Training Needs Analysis: procedural steps and pitfalls}

First the procedural steps, technical and non-technical pitfalls and possible suitable simulator models for training the TURP procedure were determined by two TURP-expert urologists and two residents using a questionnaire. By group discussion with the same urologists and residents differences in opinions were levelled and suggestions were made for which procedural steps and pitfalls the virtual reality TURPsim ${ }^{\mathrm{TM}}$ simulator might be accurate and implementable in a curriculum.

\section{Face, content and construct validation study}

This prospective study was conducted at the department of urology of three hospitals in the Netherlands (Catharina Hospital Eindhoven, Radboud University Nijmegen Medical Centre and Maastricht University Medical Centre).

A group of 66 participants, including urologists, residents and medical students participated in the study. All gave written informed consent. Participants were allotted to one of three groups based on TURP experience defined as number of performed TURP procedures: Novices $=0$, Intermediates $=1-50$, Experienced $=50<$.

All participants received a standardized introduction on PowerPoint about general anatomy of the prostate and about pathology concerning BPH. Second they were introduced to the procedural steps of a TURP, and to the working mechanism of TURPsim ${ }^{\mathrm{TM}}$ and its resectoscope. Afterwards they performed four basic tasks (visualisation, resection of medial lobe, resection of side lobes and bleeding control) and two full-procedure TURP tasks. Participants were asked to resect $50 \%$ of the prostate and to mention out loud the procedural steps during the complete TURP procedure.

After having finished all exercises, they filled out a questionnaire on demographics and 11 questions to rate the TURPsim ${ }^{\mathrm{TM}}$ on its realism and usefulness in the urological residency training curriculum. Novices were excluded from the analysis of realism and usefulness because their lack of knowledge about the pathology and TURP procedure.

\section{Simulator}

The TURPsim ${ }^{\mathrm{TM}}$ (Simbionix, Beit Golan, Israel / VirtamMed, ETH Zurich, Switzerland) is a virtual reality simulator that contains a platform with a resectoscope-robot, resectoscope, viewing monitor, laptop, and foot pedals conform the actual TURP pedals (figure 1). The viewing monitor displays the virtual reality images of the TURP procedure. The laptop 


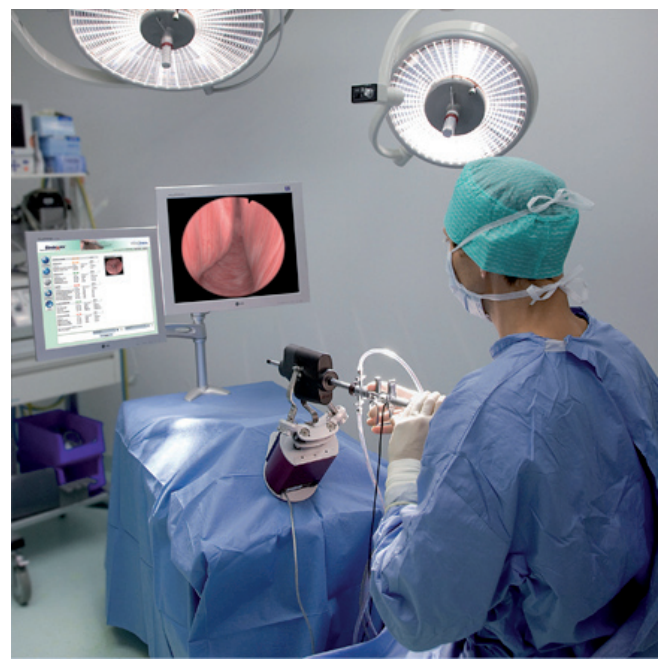

Figure 1. TURPsim ${ }^{\mathrm{TM}}$ Copyright: Simbionix Virtamed

gives an overview of the different TURP modules and reports individual performance metrics at the finish of each exercise. The simulator software enables training of 8 basic skills and 8 whole TURP procedures, which differ in prostate volume, bleeding difficulties and anatomical landmarks.

\section{Outcome parameters}

Face and content validity were measured using a questionnaire concerning participants' opinions about realism (3 items), usefulness (10 items), and overall opinion about the simulator (4 items). All items were scored on an ordinal 10-point Likert-scale; $1=$ not at all realistic/useful and $10=$ very realistic/useful.

Construct validity was measured using the outcome parameters of the training needs analysis. The simulator provided the following performance metrics; resection time, blood loss, visibility and complications as capsule resected, and lesion of the verumontanum, sphincter and bladder neck.

Additionally an observer scored the completed procedural steps during performance. This was done using a checklist, which was based on the outcome of the TNA.

\section{Statistics}

All data were processed and analysed using the Statistical Package for the Social Sciences 19.1 (SPSS inc., Chicago, USA). The Kruskal- Wallis test was performed to compare continues variables. The Mann-Whitney $U$ test was used to compare continues variables of 2 groups. Categorical variables were compared with a chi-square test. Mean or median was calculated based on their parametric or non-parametric characteristics. A p-value of $<0.05$ was considered to indicate statistical significance for all tests. 


\section{Ethics}

No ethical approval was required as there was no patient interference and the test results did not have a substantial effect on direct patient care.

\section{RESULTS}

A total of 66 participants were included in this study; 22 novices, 22 intermediates and 22 experts. Table 1 shows the demographics of these groups.

Table 1. Demographics

\begin{tabular}{lcccc}
\hline & Age in years & Sex & Dominant hand & $\begin{array}{c}\text { Experience in complete } \\
\text { TURP procedures }\end{array}$ \\
\cline { 2 - 5 } & (Median; IQR) & $(\%$ male $)$ & (\% right handed) & (Median; IQR) \\
\hline Novices & $24 ; 4$ & 41 & 91 & $0 ; 0$ \\
Intermediates & $33 ; 5$ & 68 & 82 & $20 ; 33$ \\
Experts & $48 ; 16$ & 86 & 82 & $400 ; 900$ \\
\hline
\end{tabular}

\section{Training needs analysis: TURP procedure}

The TNA resulted in a document for training the complex procedure of a TURP. In table 2 the procedural steps are given, plus the assumption of the panel whether the TURP simulator could or could not simulate (parts of) the procedural steps. Technical pitfalls that were identified by the panel were: Eye hand coordination (not looking at scope, but looking at screen), orientation of camera, tissue handling, undermining bladder neck, resection chips too short/small, loss of overview, stopping in between resection. Non-technical pitfalls were: lack of procedure-knowledge, lack of material knowledge, insufficient preparation, insufficient and incorrect communication (table 3). The TURP$\operatorname{sim}^{\mathrm{TM}}$ VR simulator used in our study covered 10 of the 22 required skills resulted from the TNA. (table 2).

\section{Face and content validity: realism and usefulness of the TURPsim ${ }^{\mathrm{TM}}$}

Only responses from intermediates $(\mathrm{N}=22)$ and experts $(\mathrm{N}=22)$ were included in the face and content analysis. Overall realism was rated 6.7 (Median: 7, range: 2-9). Haptic feedback scored 4.3 (Median: 4.5, Range: 1-8). No significant difference was found between the opinions of intermediates and experts. Examples of participant's critics were roughness of the scope, too much feedback while touching the prostate and lack of haptics during resection (figure 2). 
Table 2. Training Needs Analysis: Procedural steps

\begin{tabular}{|c|c|c|}
\hline \multicolumn{2}{|c|}{ Procedural steps } & \multirow[t]{2}{*}{ TURPsim $^{\mathrm{TM}}$} \\
\hline Preparat & ion & \\
\hline 1. & Check indication for TURP & - \\
\hline 2. & Check material including electro-surgery & - \\
\hline 3. & Check patient history, culture, antibiotic prophylaxis, use of anticoagulants & - \\
\hline 4. & Patient positioning & - \\
\hline 5. & Rectal exam & - \\
\hline 6. & Disinfection, sterile drapes & - \\
\hline 7. & Set/position material, including foot pedal & + \\
\hline 8. & Introduction cystoscope & + \\
\hline \multicolumn{3}{|c|}{ Inspection } \\
\hline 9. & cystoscopy; identification orifices, pathology, orientation, verumonatum & + \\
\hline \multicolumn{3}{|c|}{ Change instruments } \\
\hline 10. & Change to resectoscope & - \\
\hline 11. & Optional: insertion of Korth & + \\
\hline \multicolumn{3}{|c|}{ Resection of the prostate } \\
\hline 12. & Resect middle lobe. First superficial resection to get tissue feeling & + \\
\hline 13. & Resect 12 o'clock to determine midline & + \\
\hline 14. & Resect left and right lateral lobe & + \\
\hline 15. & Empty bladder in time (especially if no continuous flow system) & - \\
\hline \multicolumn{3}{|c|}{ Finishing procedure } \\
\hline 16. & Evacuate tissue & + \\
\hline 17. & Judgement of end result & + \\
\hline 18. & Haemostasis & + \\
\hline 19. & Introduction catheter and flow on catheter & - \\
\hline 20. & Check drainage via catheter & - \\
\hline 21. & Postoperative instructions to nurse & - \\
\hline 22. & Make report of procedure and fill pathological form & - \\
\hline
\end{tabular}

The usefulness of the TURP simulator as a training model for TURP skills was rated 7.6 (Median: 8, range: 2 - 10) (figure 2). Learning procedural steps was rated 8.2 (Median: 8; range 5 -10). The general score for the TURPsim ${ }^{\mathrm{TM}}$ was rated 7.3. (Median: 7; range 3-9). Intermediates gave a significant higher score than experts considering training capacity for resection skills $(p=0.047)$. Ninety- three percent of all participants considered the TURP simulator useful as training model in the urological residence program.

\section{Construct validity}

Table 4 shows an overview of novices', intermediates' and experts' performances and complications. Intermediates and experts performed the TURP procedures significantly 
Table 3. Training Needs Analysis: Pitfalls

\begin{tabular}{ccc}
\hline Pitfalls & & TURPSim $^{\text {TM }}$ \\
\hline Technical Pitfalls & + \\
1. $\quad$ Eye-hand coordination & + \\
2. $\quad$ Orientation of camera & - \\
3. $\quad$ Tissue handling & + \\
4. $\quad$ Undermining bladderneck & - \\
5. Insufficient resection of chips & + \\
6. $\quad$ Loss of overview & - \\
7. $\quad$ Stopping in /between resection & - \\
Non-technical pitfalls & - \\
8. $\quad$ Lack of procedural knowledge & - \\
9. Lack of material knowledge & - \\
10. Insufficient preparation & Insufficient or incorrect communication & \\
11.
\end{tabular}

$+=$ Pitfall can be trained and is scored by the TURPsim ${ }^{\mathrm{TM}}$;

-=Pitfall can not be trained and is not scored by the TURPsim ${ }^{\mathrm{TM}}$

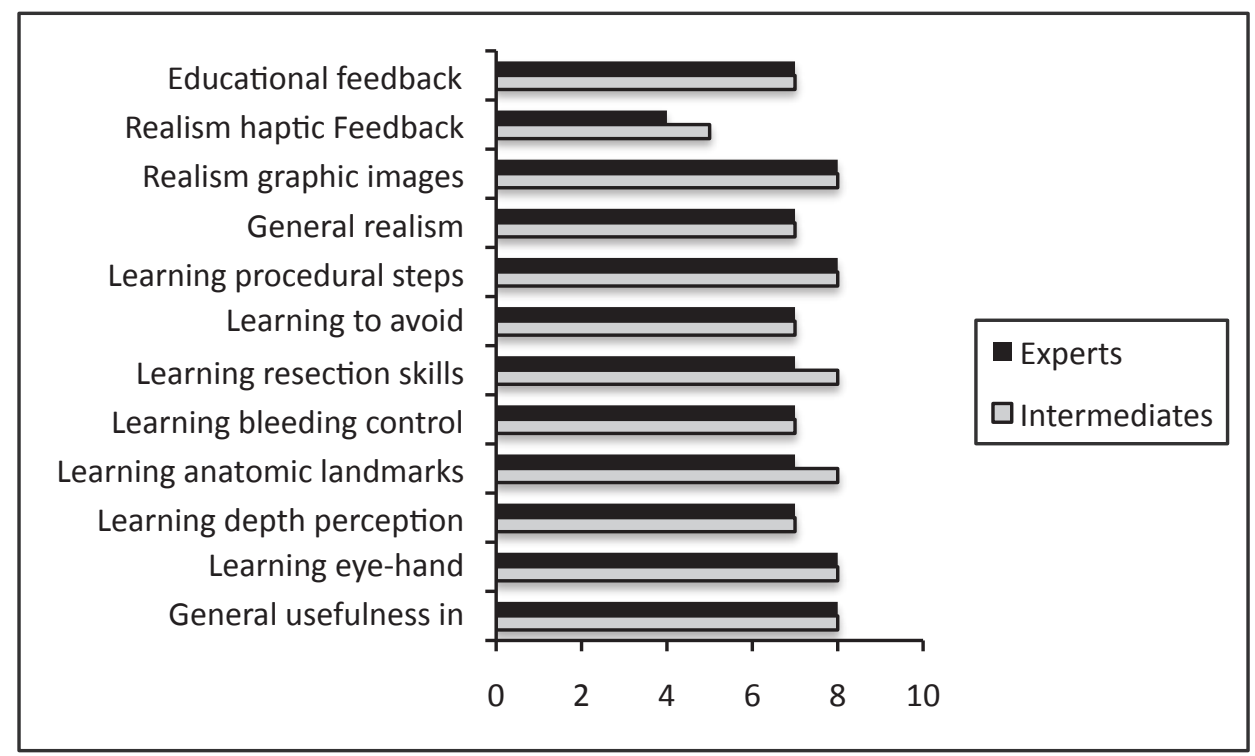

Figure 2. Opinions of intermediates and experts about realism and usefulness of the TURPsim ${ }^{\mathrm{TM}}$ $1=$ not at all realistic/useful; $10=$ very realistic/useful 
faster than novices $(p=0.001)$. Novices caused significantly more blood loss $(p=0.001)$ and significantly more sphincter cuts then intermediates and experts for the first and second TURP procedure $(p=0.001$ respectively $p=0.002)$. Other complication metrics showed no significant differences between the groups. During the second TURP procedure experts had significantly lower scores on visibility then novices and intermediates $(p=0.015)$.

Table 4. Construct validity of the TURPsim ${ }^{\mathrm{TM}}$

\begin{tabular}{|c|c|c|c|c|c|c|c|}
\hline & \multicolumn{2}{|c|}{ Novices } & \multicolumn{2}{|c|}{ Intermediates } & \multicolumn{2}{|c|}{ Experts } & \multirow[t]{2}{*}{ p-value* } \\
\hline & Median & Range & Median & Range & Median & Range & \\
\hline \multicolumn{8}{|l|}{ Performance } \\
\hline TURP 1: Time (s) & 735.0 & $394-1172$ & 414.5 & $317-712$ & 393.5 & $309-637$ & $0.001^{*}$ \\
\hline TURP 2: Time (s) & 847.5 & $455-1541$ & 475.0 & $241-866$ & 461 & $302-809$ & $0.001^{*}$ \\
\hline TURP 1: Average visibility (\%) & 95.0 & $87-99$ & 97.0 & $80-100$ & 95.0 & $89-99$ & 0.54 \\
\hline TURP 2: Average visibility (\%) & 86.5 & $61-95$ & 83.5 & $61-96$ & 77.5 & $54-92$ & $0.015^{*}$ \\
\hline \multicolumn{8}{|l|}{ Complications } \\
\hline $\begin{array}{l}\text { TURP 1: cuts into sphincter ( } \mathrm{nr} \text { of } \\
\text { times) }\end{array}$ & 11 & & 1 & & 4 & & $0.002^{*}$ \\
\hline $\begin{array}{l}\text { TURP 2: cuts into sphincter ( } \mathrm{nr} \text { of } \\
\text { times) }\end{array}$ & 8 & & 0 & & 1 & & $0.001^{*}$ \\
\hline $\begin{array}{l}\text { TURP 1: cuts into verumontamum ( } \mathrm{nr} \\
\text { of times) }\end{array}$ & 3 & & 7 & & 4 & & 0.313 \\
\hline $\begin{array}{l}\text { TURP 2: cuts into verumontanum ( } \mathrm{nr} \\
\text { of times) }\end{array}$ & 7 & & 9 & & 12 & & 0.313 \\
\hline $\begin{array}{l}\text { TURP 1: cuts into bladderneck ( } \mathrm{nr} \text { of } \\
\text { times) }\end{array}$ & 1 & & 2 & & 1 & & 1.0 \\
\hline $\begin{array}{l}\text { TURP 2: cuts into bladderneck ( } \mathrm{nr} \text { of } \\
\text { times) }\end{array}$ & 5 & & 3 & & 3 & & 1.0 \\
\hline TURP 1: Bloodloss (ml) & 63.0 & $8-205$ & 34.5 & $3-123$ & 35 & $4-102$ & $0.001^{*}$ \\
\hline TURP 2: Bloodloss (ml) & 164.0 & $46-408$ & 87.0 & $16-192$ & 116 & $57-253$ & $0.001^{*}$ \\
\hline
\end{tabular}

Kruskal-Wallis test between performances of novices, intermediates and experts

\section{Procedural steps}

No significant differences were found in performing cystoscopy during the first and second TURP procedure $(p=1.00$ resp. $p=0.37$ ) between novices, intermediates and experts.

In the resection phase of the first TURP procedure novices resected the prostate significantly more often in a different order $(p=0.028)$. In addition, they needed to return to previous lobes to finish the required $50 \%$ of the prostate resection $(p<0.001)$ (figure 3 ). No significant differences were found between the three groups with respect to performing haemostasis, evacuation of prostate tissue and judgement of the end result at the end of the procedure during the first and second TURP procedure $(p=0.22$ resp. $p=0.27$. 

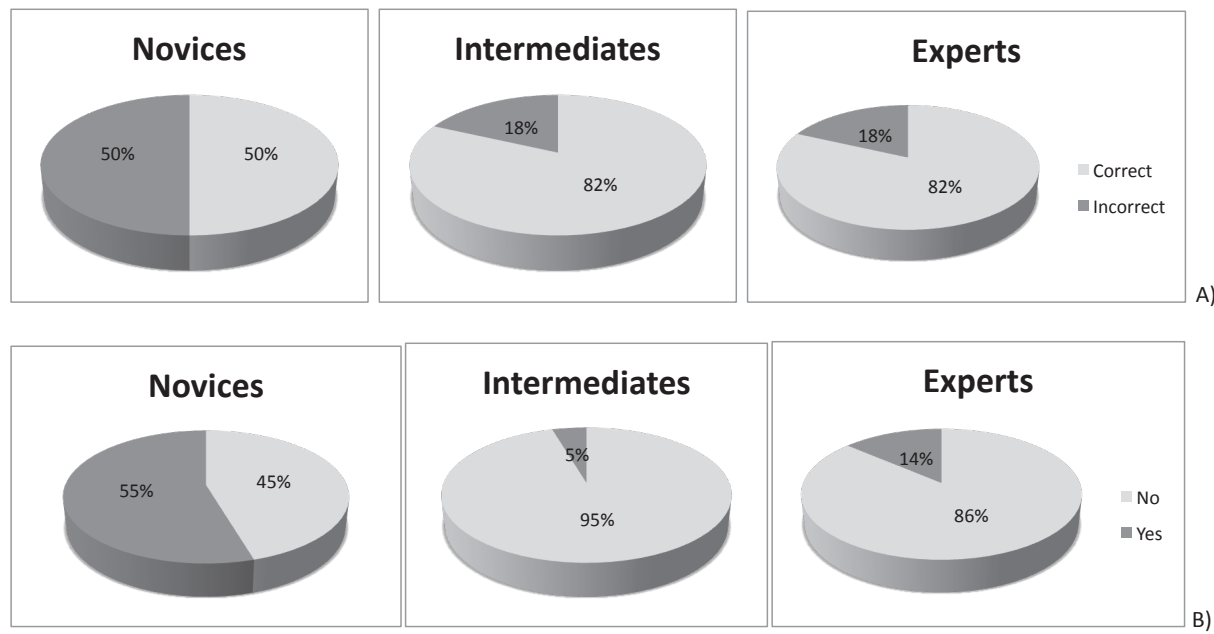

Figure 3. Performance of procedural steps in the correct or incorrect manner by novices, intermediates and experts Kruskal-Wallis test between performances of novices, intermediates and experts

A) Order of resection ( $p=0.028)$

B) Need to re-resect previous lobes to reach the required amount of $50 \%$ prostate resection $(p<0.001)$

\section{DISCUSSION}

The TURPsim ${ }^{\mathrm{TM}}$ VR simulator covered 10 of the 22 defined procedural skills and 4 out of 11 pitfalls of the TNA. Results of the present study showed face and content validity of the TURPsim ${ }^{\mathrm{TM}}$; almost $95 \%$ of the participant rated this VR-simulator as useful for the urology training curriculum for learning technical TURP-skills. Construct validity was established as discrimination in performance between different levels of expertise was shown. Significant differences were mainly found between novices and intermediates/ experts. Differences between the intermediate and the expert group were not significant, suggesting that the simulator is most suitable for trainees in the beginning of their urological residency. However, construct validity for the individual learning curve remains to be investigated in future studies.

Several types of TURP simulators have been described in the literature. ${ }^{9,18-26}$ Not all of them were validated and some of them were only early prototypes that were not further developed. Most of the validation studies of simulators for surgical skills based their outcome parameters on the metrics given by the simulator. In our study we prospectively determined outcome parameters for TURP skills training using a TNA. To define relevant parameters is of paramount importance for establishing validity of a simulator since it is desirable to evaluate only those skills that one needs to perform on patients. ${ }^{4,6}$ In our study all by TNA identified 10 skills appeared to be technical skills. To check procedural steps we were constrained to use a checklist, since the simulator does not provide met- 
rics to evaluate this. Although the simulator received appropriate rating of 8 for training procedural steps, we still recommend a mentor to give oral instructions and to judge the procedural steps.

A critical issue with any simulator is whether it accurately represents reality. Bright at al established content validity of the TURPsim ${ }^{\mathrm{TM}}$ and our results are in concordance with their results. ${ }^{26}$ As figure 2 shows, the intermediate and expert groups were consistent in their opinion that the TURPsim ${ }^{\text {TM' }}$ educational feedback and graphic images approaches real live TURP features, however haptic feedback received moderate to low ratings. Cut-off score was based on previous studies evaluating TURP-simulators. A score of 6 or higher on a 10-point scale was considered as positive.

Intermediates and experts reported a mean rating of 4.3 for haptic feedback, what is less than adequate for validity. Nevertheless, our study showed discriminative ability of the TURPsim ${ }^{\mathrm{TM}}$ for time, sphincter cuts and blood loss when comparing participants with different levels of experience. Haptic feedback alone was thus not constraining the construct validity of the TURPsim ${ }^{\mathrm{TM}}$. Haptic feedback that feels realistic has been shown to have an important role in simulation of minimal invasive surgery, but it is also known that constructing haptic feedback in surgical VR-simulators is technically difficult.27, 28 Future studies should focus on refinement of the haptic feedback device to improve simulators' training ability.

Experts appeared to resect more often with non-optimal visibility than novices and intermediates. This finding is in concordance with the results of the validation studies of the METI/CAE healthcare Surgisim by Sweet et $\mathrm{al}^{19}$ and Rashid et al. ${ }^{20}$ This phenomenon of experts continuing the procedure with more blood loss or less visibility could be explained by the fact that experts know from their experience when and where to resect safely. Additionally, our study results showed that novices perform the TURP-procedure significantly less efficient than experts since they need to re-resect lobes more often and their order of resection is frequently incorrect when compared to the procedural steps of experts. A reason for the finding that novices still resect incorrectly, despite a thorough instruction could be that they received too much information in a short amount of time. According to the cognitive load theory, the human system has a limited working memory that can hold no more than five to nine elements, and actively process no more than two to four elements simultaneously. ${ }^{29}$ The working memory only applies to new information. Long-term memory holds cognitive schemas that vary in their degree of complexity and automation. ${ }^{29}$ For novices the whole TURP-procedure, working mechanisms of the instruments, the prerequisite knowledge about the human anatomy and pathophysiology, is not yet automatic.

Apparently there is need to develop metrics integrating unconscious or automatic skills of experts with safe performances into a simulators' software for training whole procedures. Instructional design frameworks for "promoting learning" also recommended this 
statement. These frameworks assume that experts are expected to perform parts of the procedure or the whole procedure automatically, and thus to make movements more fluently and to make decisions more intuitively. ${ }^{30,31}$ From the perspective of instructional design, major advantage of simulation based training as an adjunct to clinical practice is that the trainee can practice deliberately and, most important, in a safe environment were errors can be made, until a certain level of automaticity is achieved for parts of the procedure.

Whole task training with increasing level of complexity is recommended above part task practices. ${ }^{32,33}$ However a certain level of basic knowledge is required for each procedure and thus modules to train part tasks that require a high level of automaticity are preferable. ${ }^{30,34}$ The TURPsim ${ }^{\mathrm{TM}}$ provides whole procedure with different levels of difficulty and part task practices, such as bleeding control and resection of the medial lobe.

Most of the validation studies in surgical technical skills training focus on validating individual simulators, not curricula. For example Bright et al focussed on the content and construct validation of the TURPsim ${ }^{\mathrm{TM}}$ on one module; "resection task 1" a simple task requiring resection of the median lobe during which no complications such as bleeding occur. ${ }^{26}$ In our study we attempted to use a curriculum based approach with a theoretical part and a training part. The latter is to train "part-tasks" to get used to the simulator and to train basic performances before starting to train a whole procedure. In our opinion future studies should focus more on validating individual simulators including their place in the current curriculum and involve expert opinions prospectively about required skills as first step of the validation study, using TNA to determine procedural steps and identify pitfalls. Experts should be involved in the development process of a simulator so that it can meet expectations of the intended users and to develop modules required to train relevant skills needed for practice on patients. ${ }^{23,30,35}$

The checklists resulted from the TNA could be a useful tool to give constructive feedback in clinical practice as we have shown that the simulator does not cover training abilities for all required skills in a TURP procedure. We recommend that future studies should focus on validation of checklists and cross-correlation of these checklists with inbuild performance metrics of a simulator, to evaluate its capability to use these checklists or the simulator as an assessment tool or as a constructive feedback mechanism.

Limitation of this study is that only two experts and two residents of one institution were involved in the TNA, resulting in a checklist with mainly psychomotor skills. Therefore, in future, we recommend to use a more structured method for the TNA, integrating psychomotor and cognitive skills, for example cognitive task analysis (CTA). ${ }^{36,37}$ CTA refers to a variety of interview and observation methods aimed at unravelling experts' performance of complex tasks to capture the knowledge, decision making processes and goal structures the experts rely on during task performances. ${ }^{36,37}$ In CTA it is important to identify which part of a task can be classified as "automatic", where experts 
perform tasks without conscious awareness, and which parts as "non-automatic" requiring conscious attentions, also for experts. CTA itself does not capture a single method for gaining insight in expert performances. For each procedure or training researchers should consider the most appropriate interview and observation methods and carefully define their research population, preferably guided by an educationalist who is trained in how to conduct CTA. ${ }^{30}$

The importance of a curriculum based approach for development of skills training is also underlined by Sweet et al. (2010). He described a backward design approach where defining intended and desired outcome are first steps in the process. It was also mentioned that validation and curriculum development are interdependent and a continuous process. ${ }^{35}$ In addition to this backward design approach we recommend that analysis should mainly focus on sub-skills that are non-automatic for novices and are automatic for experts since these skills are indicated to train repetitively on a simulator. ${ }^{30}$ This curriculum based approach including validity of a simulator and its value in the curriculum may narrow the gap between skillslab and clinical practice.

\section{CONCLUSION}

TNA appeared to be of paramount importance in the validation and evaluation process to determine procedural steps and to identify pitfalls. This curriculum-based approach of evaluation of the validity of a simulator may narrow the gap between skillslab and clinical practice. This study showed face, content and construct validity of the TURPsim ${ }^{\mathrm{TM}}$ and this simulator founds its place in the current urological curriculum to train basic and procedural TURP skills, especially for trainees in the beginning of their residency.

\section{Acknowledgement}

The authors would like to thank all the medical students, residents in urology and urologists who participated in this study to evaluate the TURPsim ${ }^{\mathrm{TM}}$. The authors also would like to thank Dr. Ir. Marcel van 't Veer for his statistical assistance. 


\section{REFERENCES}

1. Nestel D, Groom J, Eikeland-Husebo S, O'Donnell JM. Simulation for learning and teaching procedural skills: the state of the science. Simul Healthc. 2011;6 Suppl:S10-3.

2. Scott DJ. Patient safety, competency, and the future of surgical simulation. Simul Healthc. 2006;1(3):164-70.

3. Issenberg SB, McGaghie WC, Hart IR, Mayer JW, Felner JM, Petrusa ER, et al. Simulation technology for health care professional skills training and assessment. JAMA. 1999;282(9):861-6.

4. Zevin B, Levy JS, Satava RM, Grantcharov TP. A consensus-based framework for design, validation, and implementation of simulation-based training curricula in surgery. J Am Coll Surg. 2012;215(4):580-6.

5. Molenda M. In search of the elusive ADDIE model. Performance Improvement. 2003;42(5):3.

6. Schout BM, Hendrikx AJ, Scheele F, Bemelmans BL, Scherpbier AJ. Validation and implementation of surgical simulators: a critical review of present, past, and future. Surg Endosc. 2010;24(3):53646.

7. Kaufman RE, F.W. Needs Assessment: Concept and application. New Jersey: Educational Technology Publications Inc: Englewood Cliffs NJ; 1979.

8. van Merrienboer JJ. Training Complex Cognitive Skills: A four-component instructional design model for technical training. New Jersey: Educational Technology Publications Inc: Englewood Cliffs; 1997.

9. Schout BM, Bemelmans BL, Martens EJ, Scherpbier AJ, Hendrikx AJ. How useful and realistic is the uro trainer for training transurethral prostate and bladder tumor resection procedures? J Urol. 2009;181(3):1297-303.

10. Salas E, Cannon-Bowers JA. The science of training: a decade of progress. Annu Rev Psychol. 2001;52:471-99.

11. McDougall EM. Validation of surgical simulators. J Endourol. 2007;21(3):244-7.

12. Miano R, De Nunzio C, Asimakopoulos AD, Germani S, Tubaro A. Treatment options for benign prostatic hyperplasia in older men. Med Sci Monit. 2008;14(7):94-102.

13. Smith RD, Patel A. Transurethral resection of the prostate revisited and updated. Curr Opin Urol. 2011;21(1):36-41.

14. Sweet RM. Review of trainers for transurethral resection of the prostate skills. J Endourol. 2007;21(3):280-4.

15. Cury J, Coelho RF, Bruschini H, Srougi M. Is the ability to perform transurethral resection of the prostate influenced by the surgeon's previous experience? Clinics (Sao Paulo). 2008;63(3):315-20.

16. Kacker R, Williams SB. Endourologic procedures for benign prostatic hyperplasia: review of indications and outcomes. Urol J. 2011;8(3):171-6.

17. Kelly BD, Curtin PD, Corcoran M. The effects of the European Working Time Directive on surgical training: the basic surgical trainee's perspective. Ir J Med Sci. 2011;180(2):435-7.

18. Mishra S, Kurien A, Ganpule A, Veeramani M, Sabnis RB, Desai M. Face and content validity of transurethral resection of prostate on Uro Trainer: is the simulation training useful? J Endourol. 2010;24(11):1839-43.

19. Sweet R, Kowalewski T, Oppenheimer P, Weghorst S, Satava R. Face, content and construct validity of the University of Washington virtual reality transurethral prostate resection trainer. J Urol. 2004;172:1953-7.

20. Rashid HH, Kowalewski T, Oppenheimer P, Ooms A, Krieger JN, Sweet RM. The virtual reality transurethral prostatic resection trainer: evaluation of discriminate validity. J Urol. 2007;177(6):2283-6. 
21. Hudak SJ, Landt CL, Hernandez J, Soderdahl DW. External validation of a virtual reality transurethral resection of the prostate simulator. J Urol. 2010;184(5):2018-22.

22. Kallstrom R, Hjertberg $H$, Svanvik J. Construct validity of a full procedure, virtual reality, real-time, simulation model for training in transurethral resection of the prostate. J Endourol. 2010;24(1):109-15.

23. Kallstrom R, Hjertberg $\mathrm{H}$, Kjolhede $\mathrm{H}$, Svanvik J. Use of a virtual reality, real-time, simulation model for the training of urologists in transurethral resection of the prostate. Scand J Urol Nephrol. 2005;39(4):313-20.

24. Bach T, Geavlete B, Herrmann TR, Gross AJ. "Homemade"TUR-simulator for less than $\$ 40$ U.S.? The "Tupper" experience. J Endourol. 2009;23(3):509-13.

25. Ebbing J, Schostak M, Steiner U, Stier K, Neymeyer J, Miller K, et al. Novel low-cost prostate resection trainer-description and preliminary evaluation. Int J Med Robot. 2011; 7(3):367-73.

26. Bright E, Vine S, Wilson MR, Masters RS, McGrath JS. Face validity, construct validity and training benefits of a virtual reality TURP simulator. Int J Surg. 2012;10(3):163-6.

27. Vapenstad C, Hofstad EF, Lango T, Marvik R, Chmarra MK. Perceiving haptic feedback in virtual reality simulators. Surg Endosc. 2013;27(7):2391-7.

28. Chmarra MK, Dankelman J, van den Dobbelsteen JJ, Jansen FW. Force feedback and basic laparoscopic skills. Surg Endosc. 2008;22(10):2140-8.

29. van Merrienboer JJ, Sweller J. Cognitive load theory in health professional education: design principles and strategies. Med Educ. 2010;44(1):85-93.

30. Tjiam IM, Schout BM, Hendrikx AJ, Scherpbier AJ, Witjes JA, van Merrienboer JJ. Designing simulator based training: an approach integrating cognitive task analysis and four-component instructional design. Med Teach. 2012;34(10):698-707.

31. Van Merrienboer JJKPA. Ten steps to complex learning: A systematic approach to four complonentinstructional design. London, UK: Taylor and Francis; 2007.

32. Merrill M. First Principles of instruction. Educational Technology Research and Development. 2002;50(3):17.

33. Brydges R, Carnahan H, Backstein D, Dubrowski A. Application of motor learning principles to complex surgical tasks: searching for the optimal practice schedule. J Mot Behav. 2007;39(1):40-8.

34. Kishore TA, Beddingfield R, Holden T, Shen Y, Reihsen T, Sweet RM. Task deconstruction facilitates acquisition of transurethral resection of prostate skills on a virtual reality trainer. J Endourol. 2009;23(4):665-8.

35. Sweet RM, Hananel D, Lawrenz F. A unified approach to validation, reliability, and education study design for surgical technical skills training. Arch Surg. 2010;145(2):197-201.

36. Clark RE, Feldon DF, van Merrienboer JJ, Yates KA, Early S. Cognitive Task Analysis. In: al SJMe, editor. Handbook of Research on Educational Communications and Technology. London: Taylor and Francis Group; 2008. p. 577-93.

37. Yates KAF, D.F. Advancing the practice of cognitive task analysis; a call for taxonomic research. Theor Issues Ergon Sci. 2010;11:1464-536. 




\section{ABSTRACT}

Most studies of simulator based surgical skills training have focused on the acquisition of psychomotor skills, but surgical procedures are complex tasks requiring both psychomotor and cognitive skills. As skills training is modelled on expert performance consisting partly of unconscious automatic processes that experts are not always able to explicate, simulator developers should collaborate with educational experts and physicians in developing efficient and effective training programmes.

This paper presents an approach to designing simulator based skill training comprising cognitive task analysis integrated with instructional design according to the fourcomponent/instructional design model. This theory-driven approach is illustrated by a description of how it was used in the development of simulator based training for the nephrostomy procedure. 


\section{INTRODUCTION}

It is generally acknowledged that a "training needs analysis" should be one of the first steps in the development of educational interventions, including simulator based training, ${ }^{1,2}$ but other aspects that need to be considered in designing and developing simulator based training of complex surgical skills have received scant attention in the literature. Studies evaluating surgical simulators generally emphasise either psychomotor skills or theoretical procedural knowledge, ${ }^{2-7}$ but surgical procedures rely not solely on "how to" perform skills appropriately, they also require appropriate decision making at certain points during the procedure..$^{89}$ Although it seems clearly preferable for surgical simulators to be aimed at integrated training of psychomotor and cognitive skills, systematic methods for designing such simulators remain to be developed.

The need for integrated simulator based training has become more urgent today, now that the recent introduction of competency-based programmes in postgraduate specialty training, the restrictions of residents' working hours and financial constraints are all contributing to a decrease in the opportunities for "learning by doing" in the clinical workplace, ${ }^{9-11}$ the cornerstone of the traditional master-apprentice model characterised by skills acquisition starting with observation, followed by imitation under gradually fading supervision and culminating in independent performance. During this process trainees learn to integrate manual and cognitive skills, the latter including the ability to take appropriate decisions at appropriate points during a procedure. Nonetheless, when experts are asked to explain their performance in detail, it seems quite difficult for them to identify decision moments with any precision. ${ }^{12}$ Simulators could complement master-apprentice learning by explicitly pinpointing such moments, thus enabling integration of psychomotor and cognitive training for learning surgical tasks. ${ }^{2,13}$

Surgical tasks range widely from simple procedures, for example removing skin lesions and closing wounds, to highly complex surgical tasks such as laparoscopic removal of the prostate. The level of task complexity has been defined by the number of actions (either mental actions or movements) and the interactions between them; the more interactions, the more complex the task. ${ }^{14,15}$ Both mental actions and movements related to complex surgical tasks should be carefully identified before implementing in training programs. $^{16}$

Development of simulator based training should preferably start with a structured analysis of the complex procedure to be simulated using 'cognitive task analysis' (CTA), a generic term referring to a variety of interview and observation methods aimed at unravelling experts' performance of complex tasks in order to capture the knowledge, thought processes and goal structures experts rely on during task performance. ${ }^{12,17}$ Before this information can be used in developing simulator based training, it needs to be translated into instructional design focusing primarily on the identification, ordering 
and presentation of information about a skill in a blueprint for training that can help learners acquire skills in a coordinated and integrated manner. ${ }^{12}$

In learning complex surgical tasks trainees can either practice according to a 'wholetask approach' or a 'part-task approach'. In a whole-task approach, learners will typically practice simple to complex versions of the whole task; in a part-task approach, the whole task is divided into several parts and learners practice the parts apart from each other. Which method to use depends on the complexity of the task: For simple tasks with few interactions between actions a part-task approach is more effective, whereas for complex tasks with many interactions between actions a whole-task approach is more effective. For example, Brydges et al. found that trainees learning complex tasks according to the whole-task approach reached significantly better outcomes than trainees who practiced according to a part-task approach. ${ }^{14}$

One of the most extensively researched systematic models for instructional design is the four-component instructional design model (4C/ID) developed by van Merriënboer (1997), which differs from traditional instructional design - where tasks are deconstructed into parts to be learned separately before being reassembled for whole task practice - by proposing that learners should perform skills of increasing complexity as whole tasks comprising four interrelated components: scenarios, supportive information, just-in-time (JIT) information, and part-task practice. ${ }^{12,18,19}$

In this article we present an approach combining CTA and the 4 C/ID model to create a blueprint for simulator based surgical training, illustrating this approach by describing how we applied it in designing simulator based training for a complex procedural skill, the nephrostomy procedure.

No ethical approval was required for this study as there was no involvement of patients and no substantial effect on direct patient care.

\section{WHAT IS A NEPHROSTOMY PROCEDURE}

Urine production is one of the major functions of the kidney, and the renal system is composed of calices where urine is drained into the collecting system before moving down into the bladder. Some of the multiple scenarios causing obstruction of the renal system require an intervention like the nephrostomy procedure, ${ }^{20}$ a common procedure in urology for gaining direct renal access, from the skin into the kidney, for urinary drainage using a nephrostomy catheter. Before the catheter is introduced, access from the skin into the kidney should be obtained with a hollow needle and guidewires. The procedure is ultrasound guided and conducted by a urologist or a radiologist, generally under local anaesthetic. The severe discomfort experienced by patients when the procedure takes too much time to complete or is complicated by events like the need 
for multiple punctures or haemorrhage emphasises the need for training to acquire the appropriate skills before performance in real patients so as to prevent complications and reduce performance time. Skilful performance of nephrostomy prevents damage to renal vessels, surrounding organs and the urinary collecting system as well as major complications, such as damage to the renal vessels causing massive haemorrhage, bowel perforation, pleural perforation and damage to other surrounding organs. Complication rates of performance by experienced specialists vary from $1-4 \%$, and correct choice of calyx and correct performance of the procedure can lower the risk of complications. ${ }^{20,21}$

\section{HOW TO CONDUCT A CTA?}

On the basis of the principle that performance of any complex task requires a combination of psychomotor and cognitive skills, CTA is aimed at unravelling how experts make decisions while performing a complex task. ${ }^{1}$ Because novices are by definition still learning, their task performance is likely to be slower, clumsier and more prone to error than that of experts. Not only do novices' manual skills fall short, due to their lack of familiarity with all the steps of a procedure, each step requires conscious and deliberate decision making. Experts, by contrast, perform procedures more fluently and with greater ease having automated many of the steps to the extent that performance requires little if any conscious effort. It is precisely because experts are no longer conscious of every step of their performance that they, when asked to elaborate on the steps of a certain task, have difficulty identifying the points where decisions are made. ${ }^{12}$ In CTA it is therefore important to identify which parts of a task can be classified as 'automatic', i.e. being generally performed by experts without conscious awareness, and which parts as "nonautomatic', requiring conscious attention also from experts. We will describe the CTA method we used to analyse the nephrostomy procedure.

\section{CTA OF THE NEPHROSTOMY PROCEDURE: A PRACTICAL APPROACH}

In order to unravel the cognitive steps of a nephrostomy procedure, we used a two-stage CTA approach. The first stage comprises questionnaires to identify the main steps of the procedure and the second stage was a semi-structured interview to unravel automatic and non-automatic sub steps.

- Participants: Four urologists all from the same hospital were asked to complete a questionnaire, and eight expert urologists from seven different hospitals were invited to participate in a semi-structured group interview. All participants performed 
nephrostomy procedures on a regular basis. Two urologists who completed the questionnaires, were also included in the semi-structured interview; however they were unaware of the results before attending the semi-structured interview. The eight urologists were all male, had been practising urologists for a mean period of twelve years (range 5-25) and had performed a median number of 175 nephrostomy procedures (range: 5 -2000).

- Method: The four urologists were asked to complete a questionnaire about the sequential steps of nephrectomy, asking them to identify the steps and list for each step which instruments and materials were needed and which complications could occur. After the questionnaires were analysed, a second round of the CTA was held aimed at reaching agreement on the various elements of the procedure based on the results of the analysis and the literature. A third round was not required as consensus was reached. The eight urologists took part in a four-hour expert meeting starting with an introduction of the educational principles of skills training and an explanation of the purpose of the meeting, followed by a semi-structured group interview conducted by an educational expert, a researcher and an expert urologist. A scenario of a non-acute patient requiring a standard nephrostomy guided the questionnaire, the initial results of the questionnaire, supported by five main questions (table 1) and any new questions prompted by issues brought up during discussion with the experts, guided the interview. Each step was analysed in the order shown in Figure 1 to identify automatic and non-automatic sub-steps according to their own experience. For each step and sub-step all questions had to be answered, and the step had to be classified as automatic or non-automatic. Not until all sub-steps had been dealt with and consensus was reached, did the interview move on to the next step of the procedure. If consensus was not achieved, the differing opinions were recorded in writing.

Table 1. Five questions that guided the semi-structured interview

1. Please identify all sequential steps of the nephrostomy procedure?
2. $\quad$ Which steps can be considered as automatic and which can be considered as non-automatic? (follow
3. $\quad$ Which complications are likely to occur?
4. $\quad$ How do you prevent the complications mentioned in question 4 ?
5. Please report 5 cases with different difficulty levels.

- Data analysis and interpretation: The CTA was documented in a flowchart and the interview was recorded using a digital voice recorder. The data were analysed by the researcher who additionally described the decision making process leading to the categorisation of (sub-)tasks as automatic or non-automatic. 


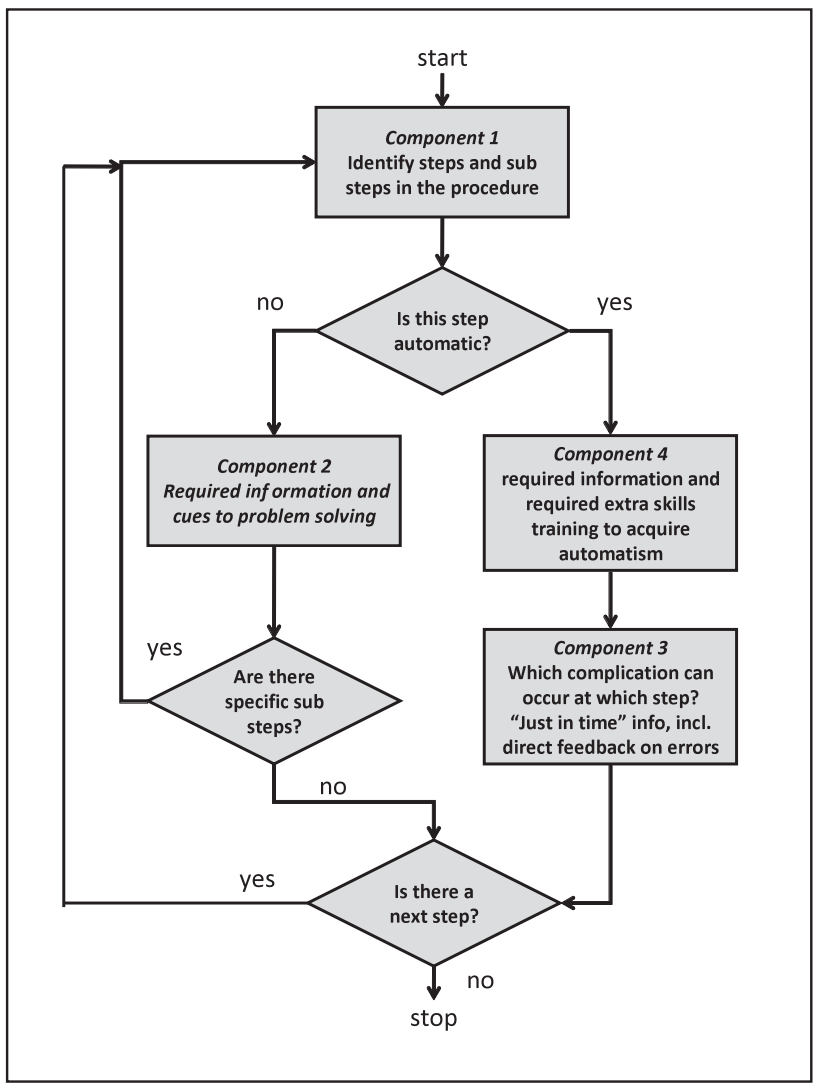

Figure 1. Flowchart of CTA

- Results: The first column in Figure 2 shows the sequential steps of the nephrostomy procedure arrived at after the two stages of the CTA. It proved impossible to reach consensus on the place in the sequence of 'incision in the skin' and 'local anaesthetics', but extensive discussion resulted in agreement on the sequence of steps shown in Figure 2. During the interview, several sub-tasks with high levels of automaticity were identified. The following sub-tasks were classified as requiring conscious attention and thus classified as non-automatic: 'Following the tip of the needle continuously during insertion from the skin into the renal system' and 'Introducing the nephrostomy catheter over the guidewire into the renal system'. After a thorough discussion consensus was reached among all eight urologists, considering the identification and classification of automatic and non-automatic steps.

Figure 2 shows a flowchart presenting details of the sequential steps and sub-steps of the nephrostomy procedure resulting from the interview. All steps were classified as either automatic or non-automatic. 


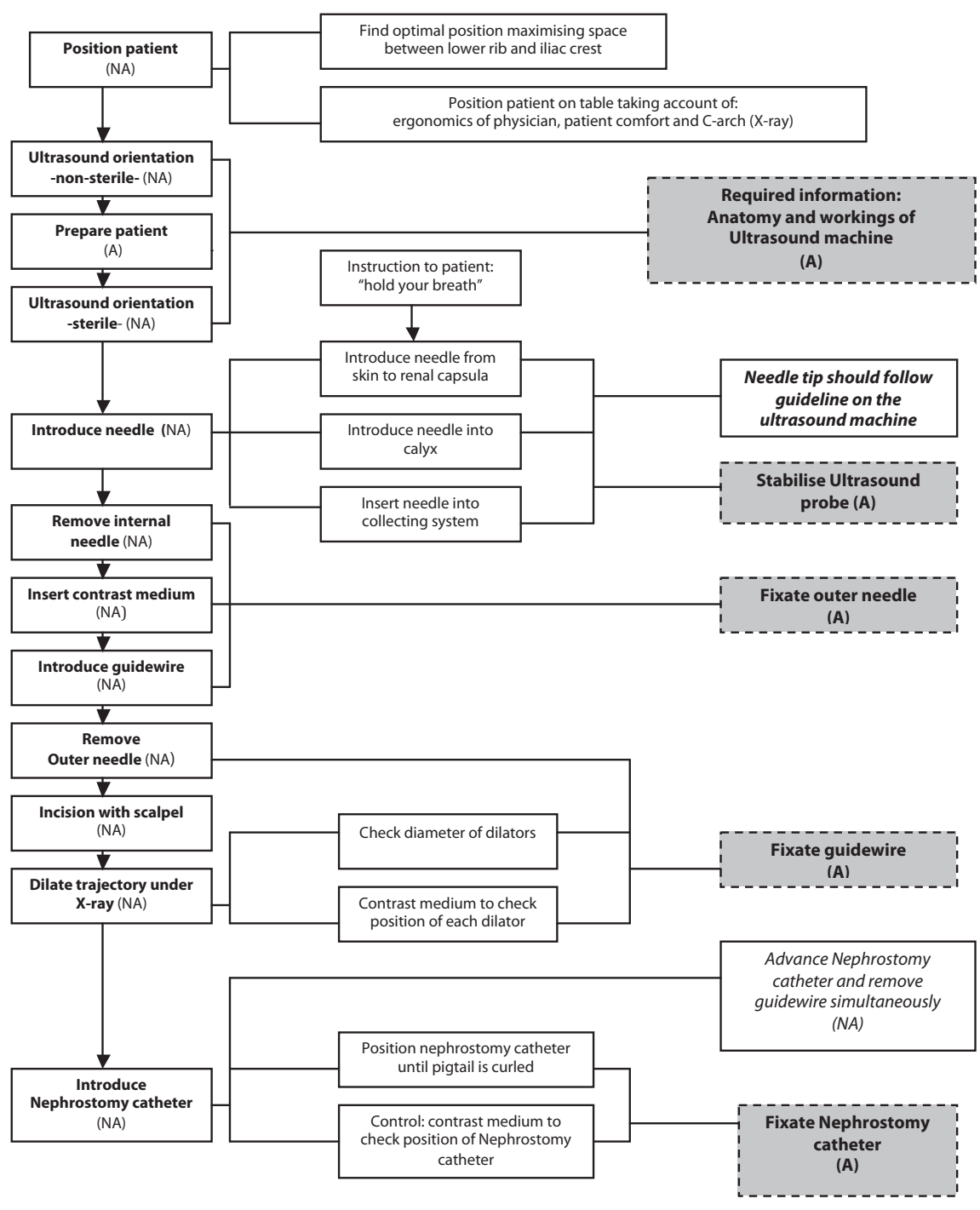

Figure 2. Flowchart of tasks and sub-tasks of the nephrostomy procedure.

Notes: All tasks are classified as automatic (A) or non-automatic (NA). All steps of the left vertical sequence are considered main steps, of which some are differentiated into sub-steps, because they were first considered as non-automatic. The tasks in the grey boxes require high automaticity according to the experts. 


\section{HOW TO DESIGN SIMULATOR BASED TRAINING ACCORDING TO THE 4C/ID-MODEL}

Developed by van Merriënboer (1997), the '4 C /ID model' is one of the most extensively developed instructional design models. ${ }^{12}, 19$ It is aimed at creating whole-task training integrating different competences, based on the assumption that effective training consists of four interrelated components: scenarios, supportive information, JIT information, and part-task practice. ${ }^{12,19}$

- Scenarios are whole tasks of increasing levels of difficulty.

- Supportive information is the knowledge prerequisite for performing a task, particularly the skills classified as non-automatic, and it is divided into descriptive information, prescriptive information and cognitive feedback. Descriptive information relates to theoretical knowledge, such as knowledge about the pathophysiology of post-renal problems and human anatomy. Prescriptive information consists of ways to tackle a problem, such as systematic performance of a procedure. Cognitive feedback helps learners reflect on the quality of their acquired supportive information. According to the $4 \mathrm{C} / \mathrm{ID}$ model, trainees need more, or more detailed, supportive information as scenarios increase in difficulty.

- JIT information provides 'how-to' instructions and direct feedback on the performance of skills classified as automatic and with potentially high impact complications in the case of failure, such as haemorrhage or mortality.

- Part-task practice can be developed for skills that have to be trained to a high level of automaticity and can potentially benefit from isolated complementary training.

\section{DESIGNING NEPHROSTOMY TRAINING: A PRACTICAL APPROACH}

We illustrate the development of a design for simulator based surgical skills training according to the 4 C/ID model by describing how we arrived at a blueprint for simulator based nephrostomy training in a process in which CTA and the 4 C/ID model were integrated.

- Participants: The same eight urologists that participated in the semi-structured interview.

- Methods: In order to elicit information for use in the instructional design, two additional questions relating to error analysis and scenario design were included in the semi-structured interview, requesting the urologists to create a variety of scenarios representing at least two levels of difficulty. Information gathering for the other three 
components of the 4C/ID model was integrated in the semi structured interview as is shown in Figure 1. Component 1 requires scenarios of different levels of difficulty; component 2 requires supportive information for non-automatic skills; component 3 requires 'how-to' instructions, particularly direct feedback on performance of automatic skills ('JIT information') and component 4 requires 'part task practice' for sub-tasks to be trained to a high level of automaticity.

\section{Difficulty level 1: (standard procedure and handling of different anatomical features \\ Trainees learn how to puncture a mildly dilated renal system $(1-2 \mathrm{~cm})$ in a patient with normal anatomy \\ Supportive information: (Descriptive information) \\ Before training, trainees must have knowledge, from anatomy textbooks, about normal anatomy of the kidney and surrounding organs and blood vessels. They must also know how to use the ultrasound machine and how to handle the probe. \\ Supportive information: (Prescriptive information)) \\ - The trainee must know the correct procedure $\rightarrow$ A video tape shows correct performance of the nephrostomy procedure on the simulator (based on the results of the CTA) \\ - All scenarios can include different combinations of human features (obese, non-obese et cetera) \\ - Guidance as to which step or sub-step should be taken next (guidance fades as trainees progress in training)}

\begin{tabular}{|c|c|c|}
\hline $\begin{array}{l}\text { Scenario } 1.1 \\
\text { Trainee learns how to puncture left } \\
\text { and right kidney in the upper, mid } \\
\text { and lower pole of the renal system } \\
\text { (Scenarios vary in combinations of } \\
\text { human features and can be offered } \\
\text { with or without time pressure) }\end{array}$ & $\begin{array}{l}\text { Direct feedback: (JIT-information) } \\
\text { If an error occurs: } \\
\text { Show trainee an error sign and identify } \\
\text { error. Error sign should be shown if one of } \\
\text { the following events occurs: } \\
\text { - haemorrhage } \\
\text { - puncture of other organ } \\
\text { - kidney is not punctured } \\
\text { - guidewire is not fixated and luxates } \\
\text { - maximum radiation time is exceeded } \\
\text { - performance time is exceeded }\end{array}$ & $\begin{array}{l}\text { Part-task practice } \\
\text { fixating one hand and } \\
\text { performing an exercise } \\
\text { with the other hand } \\
\text { (under time pressure) } \\
\text { Follow the tip of the } \\
\text { needle in a "misty } \\
\text { environment" } \\
\text { (simulating echoic } \\
\text { fatty and non-echoic } \\
\text { fatty tissue) }\end{array}$ \\
\hline $\begin{array}{l}\text { Scenario } \mathbf{1 . 2} \\
\text { Trainee learns how to puncture left } \\
\text { and right kidney in upper, mid and } \\
\text { lower pole of the renal system while } \\
\text { the system is obstructed by a stone } \\
\text { (Scenarios vary in combinations of } \\
\text { human features and can be offered } \\
\text { with or without time pressure) }\end{array}$ & $\begin{array}{l}\text { Direct feedback: (JIT-information) } \\
\text { Same as Scenario } 1.1\end{array}$ & $\begin{array}{l}\text { (this could take place } \\
\text { outside patient doctor } \\
\text { context, just to acquire } \\
\text { bimanual competence) }\end{array}$ \\
\hline $\begin{array}{cl}\text { Supportive Information (cognitive } \\
-\quad \text { Trainees receive cognitive fe } \\
\text { punctures, errors) } \\
\text { - Trainee receives information }\end{array}$ & edback) & ancetim \\
\hline
\end{tabular}

Figure 3. Blueprints for high fidelity simulator based training of nephrostomy, developed using CTA and 4C/ID model in an integrated manner. 
- Data analysis and interpretation: The data from the interview were used as input for the design of a blueprint comprising the four components.

- Results of the training design according to the 4C/ID model: We analysed the results of the interview and allocated the answers from the interview to one of the four components. The final result was a blueprint consisting of different simulator training scenarios (Figure 3).

\section{Scenarios}

The semi structured interview resulted in six scenarios offering different levels of difficulty depending on different combinations of standard anatomical features (Table 2) and on the presence or absence of time pressure. Puncture, for example, is more difficult in a morbidly obese patient compared to a non-obese patient, because of the distance between the skin and the kidney. If 'non-echoic' fatty tissue is present, the scenario becomes even more difficult. With a septic patient it is urgent that a procedure should be performed accurately and within the shortest possible time. Given these variations between patient cases, it is advisable to design training options for the procedure with and without time pressure. The six scenarios involved patients with: dilatation of the renal system, kidney stones in the renal system, scoliosis, cystic kidneys, medullary sponge kidney and horseshoe kidney.

Table 2. Case variation: Human features

\begin{tabular}{lll}
\hline & Easy & Difficult \\
\hline Kidney & Fixed & Mobile \\
Fatty tissue & Echoic & Non-echoic \\
Distance between skin and kidney & Small & Large \\
Surrounding organs & No other organs in trajectory & Other organs in trajectory \\
Renal calyx of preference & Lower pole & Upper pole \\
Scar tissue & No scar tissue & Scar tissue \\
\hline
\end{tabular}

\section{Supportive information}

The urologists unanimously agreed that trainees should have knowledge about normal human anatomy and its variations as well as about how to obtain optimal ultrasound images. The simulator should indicate whether skills and sub-skills are performed correctly during training. When the trainee starts a scenario of a higher level of difficulty, the simulator should provide more or more detailed supportive information. For the scenario of a patient with serious scoliosis, for example, the trainee must have knowledge about the differences in anatomy and organ position compared to a patient without scoliosis. 


\section{JIT information (error analysis)}

All urologists agreed that the potential major complications of nephrostomy were penetration of the needle into another organ (colon, spleen, liver or lung) or a blood vessel, whereas minor errors were situations that might result in failure of the procedure, but with a re-procedure still remaining an option (Table 3). All urologists agreed that trainees must learn to recognise complications and how to handle them. In the cases of major haemorrhage, for example, trainees should immediately intervene by proceeding to open surgery in the operating theatre. All information resulting from the error analysis was suitable for use as JIT information in the blueprint.

Table 3. List of possible errors during nephrostomy procedure mentioned by the experts

\begin{tabular}{|c|c|}
\hline \multirow[t]{2}{*}{ Major } & - Penetration of other organ: liver, spleen, colon, renal vessels \\
\hline & - Penetration of renal system causing leakage \\
\hline \multirow[t]{7}{*}{ Minor } & - Taking wrong trajectory and entering another calyx \\
\hline & - Taking wrong trajectory and puncture of lower rib \\
\hline & - $\quad$ Puncture outside the kidney, without causing trauma to surrounding tissue/organs \\
\hline & $\begin{array}{l}\text { - Insecure fixation of the outer needle or inaccurate removal of the inner needle possibly } \\
\text { causing dislocation of all instruments and fall outside the renal system }\end{array}$ \\
\hline & $\begin{array}{l}\text { - Insecure fixation of the guidewire while dilating or introducing the nephrostomy catheter } \\
\text { potentially causing dislocation of all instruments and fall outside the renal system }\end{array}$ \\
\hline & - Excessive duration of procedure increasing risk of sepsis \\
\hline & - $\quad$ Frequent and excessive duration of radiation \\
\hline
\end{tabular}

\section{Part task practice}

The flowchart showed that training of all bimanual skills, with one hand being fixated while the other hand is used to perform the skill, was considered important and suitable for additional, separate 'part task practice'. The semi-structured interview yielded the following skills for which 'part task practice' was considered useful: puncture a mobile kidney, puncture a kidney with scar tissue and handle different human features (Figure 3).

\section{REFLECTION ON THE APPROACH TO DESIGNING SURGICAL TRAINING INTEGRATING CTA AND 4 C/ID}

In a review, Hall et al. ${ }^{9}$ underlined the link between surgery and cognition by pointing out the importance of using educational tools in surgical training to reduce learning curves of new procedures.

To identify different steps in a procedure, Jonassen, Tessmer and Hannum ${ }^{22}$ describe 'behavioural task analysis', which focuses mainly on observations of task performance. A 
clear disadvantage of this approach for analysing experts' performance and goal structures is that results are based on the observers' interpretation of the technical and observable aspects of performance only. In this study, we aimed to identify automatic and non-automatic steps that may also include non-observable decision-making processes, thereby making the approach described by Jonassen et al. less suitable. CTA covers both cognitive and technical skills, offering a broad range of methods to gain knowledge and thus makes it appropriate for different research purposes, aimed to identify conscious and unconscious skills.

An alternative theory for the process of instructional design is presented by Gagné and Merrill. For situations where integrated learning objectives must be reached, 'enterprises' (cf. complex tasks) should help learners to develop the necessary skills in a wholetask approach. ${ }^{23}$ Similar to the 4 C/ID model, instructional design according to Gagné aims to develop multi-objective lessons or whole-task practices; and in both theories, the learning tasks comprise both cognitive skills and technical skills to support real-life task performance. An advantage of the 4C/ID method above Gagné's theory is, however, that it offers a format to elicit knowledge, which can be subsequently translated into a training blueprint for non-experts. ${ }^{19}$

Different from studies using a single-theory approach, in our study to design simulator based surgical skill training we researched a systematic approach in which CTA and $4 \mathrm{C} / \mathrm{ID}$ were used in an integrated manner. This approach offered the additional benefit of enabling us not only to identify the required psychomotor skills but also to unravel and make explicit experts' intuitive decision making, something that is generally very difficult for experts to achieve without support, and which resulted in the identification of concrete points of decision-making during the procedure, thereby making these cognitive skills amenable to training. Identification of highly automatic components is of particularly great importance, as it enables inclusion in the training blueprint of separate practice opportunities for these components. The identification of automatic components in the procedure was based on the results of the semi-structured interview.

When CTA is fully integrated in the instructional design process, optimal use can be made of its results. A major benefit of this integrated approach is that it yields a blueprint for scenarios that is expected to be easily understood by engineers without any medical or educational background who can use it in building a simulator. However, whether engineers will actually be able to accurately interpret the blueprint and use it to inform simulator design will have to be investigated in further studies. Since the integrated design method we used is generic, it can be used in designing any type of surgical skill training.

A drawback of CTA is the considerable time investment involved in an expert meeting lasting several hours preferably attended by experts from different hospitals. It seems likely that more complex procedures, radical prostatectomy for example, will require 
several meetings as well as observational studies to analyse all the steps. Semi-structured interview was supposed to be most appropriate for the purpose of this study, identifying not only experts' observable actions but also intuitive decision-making processes during the nephrostomy procedure by recalling their own experience. Using tools such as video or stimulated recall as an alternative would narrow the scope of this study because the urologists are then asked to analyse a single, idiosyncratic procedure. In addition, offering a video could interfere with the identification of steps that are performed by intuitive decisions.

As we stated previously, CTA is a generic term covering a variety of interview and observation methods, and the semi-structured group interview we used should definitely not be regarded as the only method possible for gaining insight. Researchers should consider other interview and observation methods and carefully define their research population as well, to find the most time-efficient method for each distinct research purpose.

In this study different levels of difficulty were identified by consensus in expert opinions, resulting from a single item in the CTA process. Focussing on each difficulty level, it would be interesting to investigate if each level contains similar automatic and nonautomatic steps. Imagine an emergency situation, hypothetically this situation requires other automated steps or maybe less automated steps than a non-emergency situation. We recommend future studies to focus on CTA within different levels of difficulty of a single surgical procedure.

Although at first sight the 4 C/ID model may appear to be primarily theoretical and not directly useful for translating expert performance into a training design, the integrated use of CTA and the 4 C/ID model facilitated incorporation of the information from CTA into the blueprint. Interestingly, the information that emerged from the error analysis was eminently suitable for the 'just-in-time information' component of the 4C/ID model.

Finally, scenarios for medical skills training are somewhat different to scenarios used in other disciplines. In aviation training centres, for example, pilot trainees also learn how to adapt to different (flight) situations, but aviation scenarios enable trainees to train in a simulator that is identical to the airplane they will fly after training, ${ }^{24,25}$ whereas physicians perform skills in patients that are not identical but each present with their own unique anatomy and pathology. Consequently, scenario differentiation in medical skills training should focus not only on differences in pathology, but also on differences between individual patient characteristics. In our analysis several physical characteristics were identified and the blueprint provides varying levels of difficulty for different features. 


\section{DISCUSSION AND CONCLUSION}

We have described how CTA integrated with the 4 C/ID model can be used to design simulator based training for complex medical procedures, illustrating this with our experiences in designing a blueprint for simulator based training using the integrated approach in a semi-structured group interview with expert urologists. The final blueprint that resulted can be used by simulator developers, software engineers and even simulator users, when the provided simulator comes with a training development kit.

Obviously, a blueprint for training is not an end in itself, but should lead to the realisation of a simulator, for which this paper does not provide guidelines as it lies in the hands of simulator developers. Once developed, however, the simulator will have to be evaluated to determine whether it meets the requirements of the blueprint, and more importantly still, the effectiveness of the simulator has to evaluated. Several studies have proven that CTA based training is substantially more effective than non-CTA based training, ${ }^{26-28}$ but further studies are needed to evaluate whether simulator based training according to a design resulting from the integrated design approach of CTA and the 4 C/ ID model is an effective tool to foster novices' and residents' competency development.

Finally, we wish to underline the importance of collaboration between medical experts, educational experts and simulator developers in the process of simulator based training development. The type of blueprint we have described, resulting from CTA integrated with the 4 C/ID model, may hold the key to successful simulator development by enabling simulator designers to take on board educational principles as well as medical competences.

\section{Acknowledgement}

The authors would like to thank the eight expert urologists from; the Catharina Hospital, Radboud University Nijmegen MC, University Medical Centre Utrecht, Maastricht University Medical Centre, Atrium Hospital, Isala Clinic, who participated in pilot and semi-structured interview. The authors would also like to thank Mereke Gorsira for her editorial assistance. 


\section{REFERENCES}

1. Salas E and Cannon-Bowers JA. The science of training: a decade of progress. Annu Rev Psychol. 2001; 52: 471-99.

2. Schout BM, Hendrikx AJ, Scheele F, Bemelmans BL and Scherpbier AJ. Validation and implementation of surgical simulators: a critical review of present, past, and future. Surg Endosc. 2010; 24: 536-46.

3. de la Rosette JJ, Laguna MP, Rassweiler JJ and Conort P. Training in percutaneous nephrolithotomy—a critical review. Eur Urol. 2008; 54: 994-1001.

4. Hacker A, Wendt-Nordahl G, Honeck P, Michel MS, Alken P and Knoll T. A biological model to teach percutaneous nephrolithotomy technique with ultrasound- and fluoroscopy-guided access. J Endourol. 2007; 21: 545-50.

5. Mishra S, Kurien A, Ganpule A, Muthu V, Sabnis R and Desai M. Percutaneous renal access training: content validation comparison between a live porcine and a virtual reality (VR) simulation model. BJU Int. 2010; 106: 1753-6.

6. Mishra $S$, Kurien $A$, Patel $R$, et al. Validation of virtual reality simulation for percutaneous renal access training. J Endourol. 2010; 24: 635-40.

7. Schout BM, Ananias HJ, Bemelmans BL, et al. Transfer of cysto-urethroscopy skills from a virtualreality simulator to the operating room: a randomized controlled trial. BJU Int. 2010; 106: 226-31; discussion 31

8. Flin R, Youngson $\mathrm{G}$ and Yule S. How do surgeons make intraoperative decisions? Qual Saf Health Care. 2007; 16: 235-9.

9. Hall JC, Ellis C and Hamdorf J. Surgeons and cognitive processes. Br J Surg. 2003; 90: 10-6.

10. Brandenberger J, Kahol K, Feinstein AJ, Ashby A, Smith M and Ferrara JJ. Effects of duty hours and time of day on surgery resident proficiency. Am J Surg. 2010; 200: 814-8; discussion 8-9.

11. Parsons BA, Blencowe NS, Hollowood AD and Grant JR. Surgical training: the impact of changes in curriculum and experience. J Surg Educ. 2011; 68: 44-51.

12. Clark RE, Feldon DF, van Merrienboer JJ, Yates KA and Early S. Cognitive Task Analysis. In: al SJMe, (ed.). Handbook of Research on Educational Communications and Technology. London: Taylor and Francis Group, 2008, p. 577-93.

13. Tun JK and Kneebone R. Bridging worlds: applying the science of motor learning to clinical education. Med Educ. 2011; 45: 111-4.

14. Brydges R, Carnahan H, Backstein D and Dubrowski A. Application of motor learning principles to complex surgical tasks: searching for the optimal practice schedule. J Mot Behav. 2007; 39: 40-8.

15. van Merrienboer JJ, Kester $\mathrm{L}$ and Paas, F. Teaching complex rather than simple tasks: Balancing intrinsic and germane load to enhance transfer of learning. Appl Cogn Psychol. 2006; 20: 343-52.

16. Kneebone R. Simulation in surgical training: educational issues and practical implications. Med Educ. 2003; 37: 267-77.

17. Yates KA and Feldon DF. Advancing the practice of cognitive task analysis; a call for taxonomic research. Theor Issues Ergon Sci. 2010; 11: 1464-536.

18. Janssen-Noordman AM, Merrienboer JJ, van der Vleuten CP and Scherpbier AJ. Design of integrated practice for learning professional competences. Med Teach. 2006; 28: 447-52.

19. Van Merrienboer JJG and Kirschner PA. Ten steps to complex learning: A systematic approach to four complonentinstructional design. London, UK: Taylor and Francis, 2007.

20. Horton AR, Ratnam L, Madigan J, Munnike G and Patel U. Nephrostomy-why, how and what to look out for. Imaging. 2008; 20: 29-37. 
21. Karim R, Sengupta S, Samanta S, Aich RK, Das U and Deb P. Percutaneous nephrostomy by direct puncture technique: An observational study. Indian journal of nephrology. 2010; 20: 84-8.

22. Jonassen DT, Tessmer M andHannum E. Task analysis methods for instructional design. London, UK: Routledge, 1999.

23. Gagné RM and Merrill MD. Integrative goals for instructional design. ETR\&D. 1992; 38: 23-30.

24. Rogers JG, Gaba D. Have we gone too far in translating ideas from aviation to patient safety? BMJ. $2011 ; 342$.

25. Schwaitzberg SD, Godinez C, Kavic SM, et al. Training and working in high-stakes environments: lessons learned and problems shared by aviators and surgeons. Surg Innov. 2009; 16: 187-95.

26. Feldon DFT, Timmerman BC, Stowe KA and Showman R. Translating expertise into effective instruction: The impacts of cognitive task analysis (CTA) on lab report quality and student retention in the biological science. J Res Sci Teach. 2010; 47: 1165-85.

27. Pugh CM, DaRosa DA, Santacaterina S and Clark RE. Faculty evaluation of simulation-based modules for assessment of intraoperative decision making. Surgery. 2011; 149: 534-42.

28. Velhamos GCT, Toutouzas, K.G Sillin LF, et al. Cognitive task analysis for teaching technical skills in an inanimate surgical skills laboratory. Am J Surg. 2004; 187. 




\section{ABSTRACT}

\section{Purpose}

This study aims to provide an overview of type and frequency of musculoskeletal complaints among urologists. In addition. the urologists' knowledge about ergonomic conditions during minimal invasive urology was assessed, and they were asked how they would prefer to gain knowledge about this topic.

\section{Materials and Methods}

An online and hard copy version questionnaire was administered to urologists from different countries, mainly from Europe, performing endourology and laparoscopy.

\section{Results}

Of the 285 respondents, 245 (86.0\%) urologists experienced musculoskeletal complaints in the past 12 months and $62.1 \%$ were considered to be work related. Most common areas for chronic complaints were neck, back and shoulders. Almost 50\% of the urologists experienced chronic musculoskeletal complaints, for which endourology (OR 3.06; 95\% Cl 1.37-6.80) and laparoscopy (OR 1.70; 95\% Cl: 1.27-2.28) were significant risk factors. One third of the urologists considered their knowledge about ergonomics minimal, and $8 \%$ stated that they had no knowledge about these topics. Fifty percent of the respondents preferred to integrate information about ergonomic rules into hands-on training of urological skills.

\section{Conclusion}

High prevalence of experienced musculoskeletal complaints was found among urologists predominantly related to endourology and laparoscopy. Urologists indicate to have a lack of knowledge about ergonomics in the operation room. Hence, we recommend integration of ergonomics in hands-on training programs early in the residency curriculum to gain knowledge and awareness and hopefully to offer possibilities to prevent these complaints in the future. 


\section{INTRODUCTION}

Minimal invasive urology (MIU) endured major changes since the introduction of video assisted transurethral resections and since the introduction of the first laparoscopic nephrectomy in the early 1990s. Not only has laparoscopy become an established procedure in urological practice, also endourological procedures have undergone major improvements. In current practice i.e. flexible scopes, HD-monitors and improved camera vision are in common usage in many urological workplaces. ${ }^{1}$

Advantages of minimal invasive surgery are well known as patients experience less post-operative pain, shorter hospital stay and better cosmetic results. ${ }^{2}$ On the other hand urologists who practice minimal invasive surgery have to operate in an environment that is completely different compared to open surgery in terms of freedom of movement. For example laparoscopic surgery often necessitates a non optimal posture, where the surgeon has to work with abducted shoulders, and to stand in a static posture for a long period of time. ${ }^{2}$

Surgeons' disadvantages of minimal invasive techniques are becoming increasingly known and studies have been conducted to evaluate musculoskeletal problems experienced by surgeons practising minimal invasive surgery. ${ }^{3-7}$ Most studies resulted in a strong association of physical constraints with laparoscopy. Discomfort in neck, shoulders and back were most frequently reported by surgeons as symptoms due to minimal invasive surgery. ${ }^{7-9}$

Gofrit et al. ${ }^{5}$ reported also hand, wrist and finger pain due to hand assisted laparoscopy. Only few studies were done among urologists, ${ }^{3,5}$ and little is known about the physical well-being of urologists practicing endourology. Moreover, with the rapid development of new minimal invasive techniques, it is of major importance to increase awareness of ergonomic guidelines, for example in training programs, and thus prevent the occurrence of musculoskeletal problems at an early stage. The science of ergonomics, analyses these challenges and formulates guidelines for creating a work environment that is safe for the surgeon and the operating team. Ergonomics is defined as "the scientific discipline concerned with the understanding of interactions among humans and other elements of a system, and the profession that applies theory, principles, data and methods to design in order to optimize human well-being and overall system performance" (www.iea.cc). Awareness of these guidelines among surgeons, however, is still marginal. ${ }^{10}$ Wauben et al. ${ }^{10}$ administered a survey among surgeons in Europe, including general surgeons, gynaecologists and urologists, and reported that only $11 \%$ of the responders were aware of the ergonomic guidelines.

In this study we investigated the following research questions: 1) If urologists report musculoskeletal complaints, what type and frequency are most common? 2) To what extent do urologists consider their knowledge about ergonomic conditions during 
minimal invasive urology? 3). What is the preferred method of gaining knowledge about ergonomics according to the opinion of urologists?

\section{MATERIAL AND METHODS}

\section{Questionnaire}

An online questionnaire was developed in collaboration with an ergonomist (RHM) for the purpose of this international study on musculoskeletal complaints experienced by urologists. The questionnaire gathers information on the complaints, the potential relation with endourology and laparoscopy according to the opinion of urologists, and it assesses the urologists' knowledge on ergonomics in the operation room.

The questionnaire consisted of four parts. The first part contained 10 questions about general endourology and/or laparoscopy, general medical history and whether physical complaints were experienced during the past 12 months. Endourology was defined as transurethral resection of the prostate, transurethral resection of bladder tumour, ureterorenoscopy, percutanous nephrolithothomy. Medical history was predefined as neckhernia, backhernia, joint complaints due to traumatic event, rheumatic disease, scoliosis and an open section for other pathology in medical history of the musculoskeletal system. The second part contained three specific questions for endourology, e.g. workload, physical discomfort, the occurrence of chronic complaints which are attributed to endourology and if the urologist ever reported absence of work because of the experienced chronic physical problems. The third part contained the same questions specifically for laparoscopy. Chronic complaints were defined as musculoskeletal problems, which are experienced 6 months or longer. The fourth part consisted of three questions to assess how urologists' consider their knowledge regarding optimal ergonomic conditions for minimal invasive urology, if they consider knowledge about ergonomics in urological procedures important and how they prefer to gain knowledge on this topic. All respondents were asked to rate their knowledge regarding monitor adjustment, table height and body posture on a scale of 1 to 5 , where 1 is "no knowledge", 2 is "little knowledge", 3 is "moderate knowledge", 4 is "good knowledge" and 5 is "excellent knowledge".

Most of the questions were of a closed format, except for numerical answers such as age, length, hours of practice per week and years of practice as urologists. Answer options included rank listing and 5 point Likert scales. The questionnaire ended with extra space to give suggestions for gaining knowledge or any other remarks. 


\section{Study population}

Inclusion criteria were that the participants had to be urologists, performing endourology or laparoscopy or both procedures. Urologists who perform robot assisted laparoscopy, retired urologists, residents and nurse practitioners were excluded from the study. The online questionnaire was sent to all urologists in the Netherlands from September until November 2009. In addition, a link to the online questionnaire was published in the European Urology Today (EUT), the newsletter of the European Association of Urology (EAU) in January 2010. Also, we approached urologists personally to fill in the online questionnaire or a hard-copy version of the questionnaire during the EAU annual congress in Barcelona in 2010. For the online questionnaire, "cookies" were activated, so urologists could not respond more than once. Participants recruited during the congress were excluded if they already responded to the online questionnaire.

\section{Data analysis}

Logistic regression analysis was used to investigate whether the occurrence of musculoskeletal problems was associated with age, medical history, workload, length, hours of endourology per week and hours laparoscopy per week. The data obtained from the questionnaire were analysed using SPSS version 19.0. The cut-off point for statistical significance was set at a p-value of 0.05 .

\section{RESULTS}

A total of 285 respondents completed the questionnaire. A total of 134 Dutch urologists responded to the electronic questionnaire. The European response on the link in the EUT was 57, which was increased with 94 completed questionnaires during the EAU annual congress.

\section{Demographics}

Table 1 shows the general demographics. One hundred and five respondents exclusively performed endourological procedures and 180 urologists performed both endourology

Table 1. Demographics of all urologists $(\mathrm{N}=285)$

\begin{tabular}{ccc}
\hline & Mean & (SD; Range) \\
\hline Gender (male/female) & $265 / 20$ & $(8.0 ; 29-75)$ \\
Age (years) & 46.01 & $(8.0 ; 154-200)$ \\
Height $(\mathrm{cm})$ & 179.3 & $(0.5 ; 6.0-8.5)$ \\
Glove Size & 7.5 & $(8.0 ; 1-43)$ \\
\hline
\end{tabular}


and laparoscopy. Mean hours per week spent on endourology were 8.41 hours (Standard deviation (SD): 5.98). Mean hours per week spent on laparoscopy was 6.45 hours (SD: 8.61).

\section{Musculoskeletal complaints in the past 12 months}

We focused on eight body regions for the purpose of our study: neck, back, shoulders, arms, wrist, hands, thumbs and legs., ${ }^{3-7}$ Of the 285 respondents, 245 (86.0\%) urologists experienced musculoskeletal complaints in the past 12 months. For each of the body parts figure 1 shows the percentages of respondents that experienced musculoskeletal complaints of a certain level in the past 12 months, the levels varying from no complaints, to mild, moderate, serious, and severe complaints.

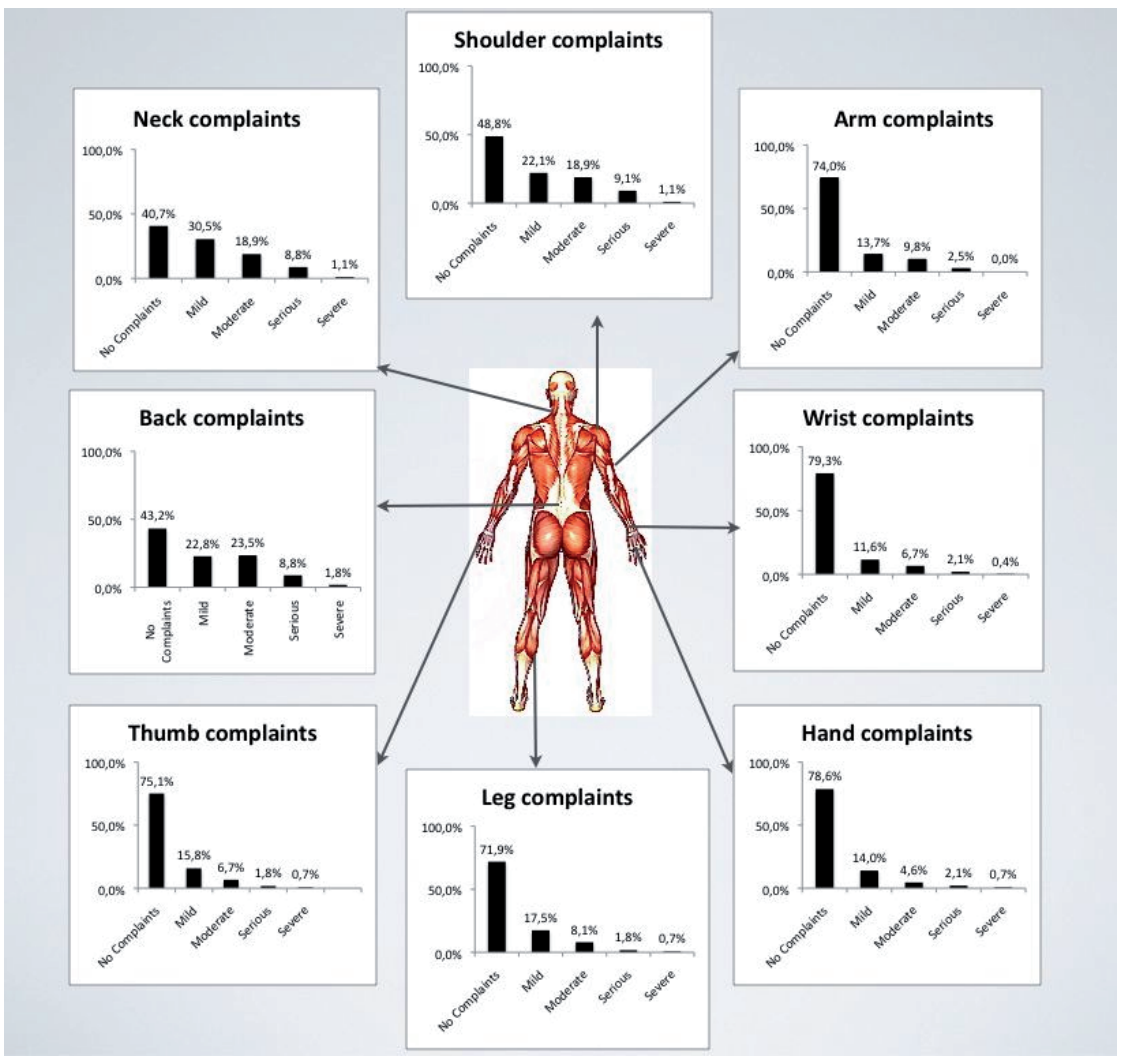

Figure 1. Musculoskeletal complaints experienced in the past 12 months 
Age and medical history were shown to be significant risk factors for reporting musculoskeletal complaints in the past 12 months. An increase of age was associated with a decrease of the risk for complaints (odds ratio $(\mathrm{OR})=0.94$ per year, $95 \% \mathrm{Cl}: 0.90-0.98$ ), and having a medical history increased the risk $(\mathrm{OR}=3.62,95 \% \mathrm{Cl}: 1.34-9.77)$. For neck $(\mathrm{OR}=2.03,95 \% \mathrm{Cl}: 1.73-3.50)$, back $(\mathrm{OR}=2.1795 \% \mathrm{Cl}: 1.27-3.72)$ and arms $(\mathrm{OR}=1.76$ : $95 \% \mathrm{Cl}: 1.01-3.07)$, only medical history was a significant risk factor.

When body regions were analysed isolated, number of hours per week spent on laparoscopy resulted in being a significant risk factor for reporting problems in thumb $(\mathrm{OR}=1.66,95 \% \mathrm{Cl}: 1.18-2.33)$ and shoulder ( $\mathrm{OR}=1.34,95 \% \mathrm{Cl}: 1.01-1.77)$. For leg problems, number of hours spent on laparoscopy per week (OR=1.52 95\% Cl: 1.09-2.11) and medical history (OR=2.06: 95\% IC: 1.18-3.60) were both significant risk factors.

One hundred and seventy-seven (62.1\%) urologists explicitly considered their complaints work related. Figure 2 shows the number of urologists who experienced musculoskeletal complaints and if they consider the complaints as a result of to endourology, laparoscopy or both procedures.

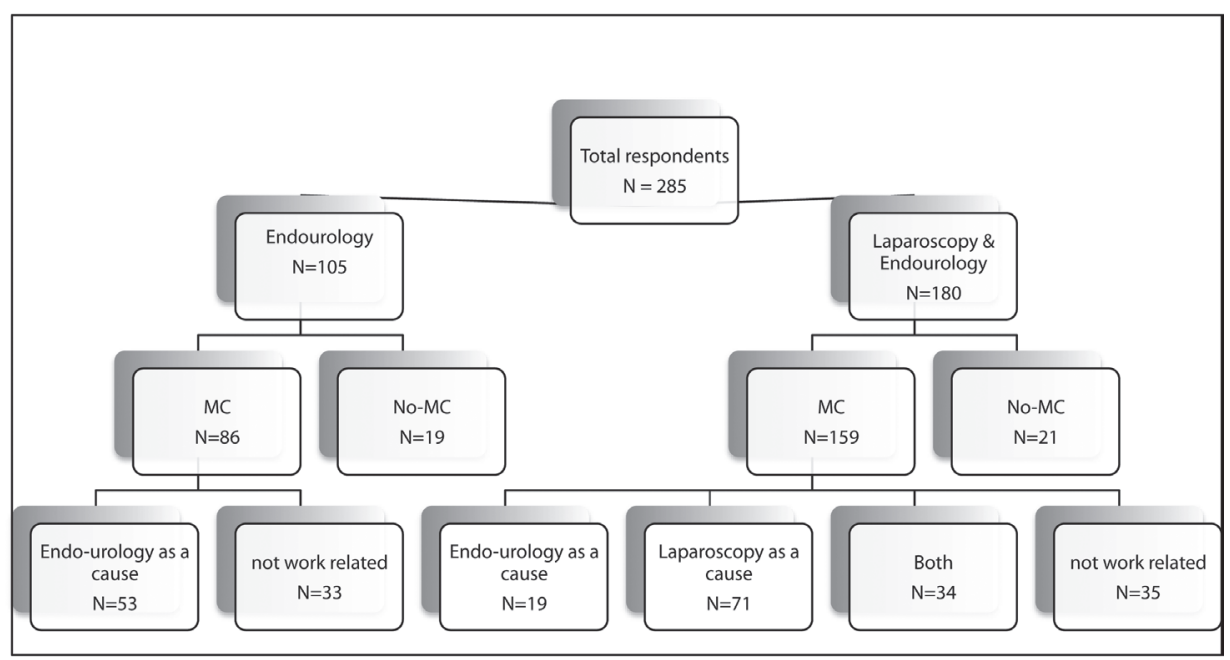

Figure 2. Reported musculoskeletal complaints in the past 12 months

\section{Chronic musculoskeletal complaints}

One hundred and thirty-nine urologists (48.8\%) experienced chronic musculoskeletal complaints. Most common areas for chronic complaints were neck, back and shoulders. Endourology (OR 3.06; 95\% Cl: 1.37-6.80) and laparoscopy (OR 1.70; 95\% Cl: 1.27-2.28) were both significant risk factor for the development of chronic musculoskeletal problems. 
From this sample of 124 urologists who performed both laparoscopy and endourology, 19 urologists $(10.6 \%)$ attributed their chronic musculoskeletal complaints to endourology, $32(17.8 \%)$ urologists attributed their complaints to laparoscopy and 50 $(27.8 \%)$ urologists thought it was due to both types of surgery.

Table 2 shows sick reports due to musculoskeletal problems attributed to endourology and to laparoscopy.

Table 2. Sick reports due to musculoskeletal problems attributed to endourology and to laparoscopy

\begin{tabular}{ccc}
\hline Reported sick & Endourology $(\mathbf{N}=\mathbf{2 8 5})$ & Laparoscopy $(\mathbf{N}=\mathbf{1 8 0})$ \\
\hline No sick reports & $263(92.2 \%)$ & $166(92.2 \%)$ \\
$<1$ week & $11(6.1 \%)$ & $9(5.0 \%)$ \\
$1-2$ weeks & $8(2.8 \%)$ & $3(1.6 \%)$ \\
$2-3$ weeks & $2(0.7 \%)$ & $1(0.5 \%)$ \\
$>4$ weeks & $1(0.4 \%)$ & $1(0.5 \%)$ \\
\hline
\end{tabular}

\section{Urologists' perception of their knowledge about ergonomics in MIU}

Although 256 (89.8\%) urologists indicated that ergonomics is important in urological practice, they considered their knowledge mainly little, moderate or good (table 3). Eight percent of the urologists reported that they did not have any knowledge regarding monitor adjustments, table height and body posture.

Table 3. Knowledge about ergonomic recommendations according to urologists' opinion

\begin{tabular}{lccccc}
\hline & No & Little & Moderate & Good & Excellent \\
\hline $\begin{array}{l}\text { Knowledge about monitor } \\
\text { height }(\mathrm{N}=282)\end{array}$ & $25(8.9 \%)$ & $80(28.4 \%)$ & $78(27.7 \%)$ & $95(33.7 \%)$ & $4(1.4 \%)$ \\
$\begin{array}{l}\text { Knowledge about table } \\
\text { height }(\mathrm{N}=290)\end{array}$ & $23(8.2 \%)$ & $65(23.2 \%)$ & $82(29.3 \%)$ & $100(35.7 \%)$ & $10(3.6 \%)$ \\
$\begin{array}{l}\text { Knowledge about body } \\
\text { posture }(\mathrm{N}=280)\end{array}$ & $24(8.6 \%)$ & $75(26.8 \%)$ & $87(31.1 \%)$ & $91(32.5 \%)$ & $3(1.1 \%)$ \\
\hline
\end{tabular}

Forty-eight percent of all respondents preferred to integrate ergonomic rules into hands-on training sessions for MIU compared to $27.9 \%$ who preferred to follow training on ergonomics specifically (Figure 3). Two respondents emphasised the importance to educate residents about ergonomics in the operation room (OR). Another urologist suggested that program directors should give attention to ergonomic rules in the operation room to their residents before starting the procedure. Several urologists suggested a combination of all methods to gain knowledge about ergonomics. Another urologist suggested inviting an ergonomic specialist to give personal advice. 


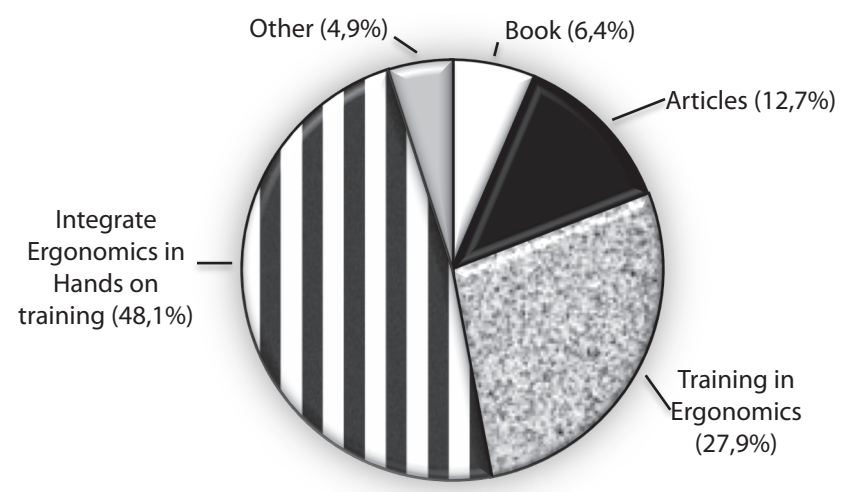

Figure 3. Urologists' preference to gain knowledge about ergonomics in the operation room

\section{DISCUSSION}

More than $85 \%$ of the urologists experienced musculoskeletal complaints in the past 12 months. Endourologists claim to experience musculoskeletal complaints almost in the same amount as laparoscopists, indicating that minimal invasive urology in general could be a risk factor for development of musculoskeletal complaints.

The response rate of 285 urologists in this study is the largest sample survey found in the literature about ergonomics in urology, and the authors consider the results of current study representative for the experienced musculoskeletal problems among urologists.

Sixty-two percent of the urologists considered their complaints work related; however our study did not find evidence that total hours per week performing endourology was a significant risk factor for reporting musculoskeletal complaints within 12 months. Laparoscopy was associated with thumb, shoulder and leg problems. The discrepancy between statistical outcome and the urologists' opinions could be explained by the fact that case load and especially mean hours per procedure are probably more important variables than total hours per week. ${ }^{6}$

When compared to other occupations our findings for neck (59.3\%) and shoulder (51.2\%) complaints were higher than reported for light workload employees (44\% and $42 \%$ respectively), i.e. administrators, service and sales workers, and interestingly also for heavy workload employees ( $39 \%$ and $43 \%$ respectively), i.e. agriculture workers, fishery workers and trade workers in New Zeeland..$^{11,12}$ From the perspective of general occupations, this form of surgery can thus be considered as one with heavy workload. When compared to similar studies assessing musculoskeletal complaints due to minimal invasive surgery our results are consistent., ${ }^{3,9,9,13,14}$ Comparison with other studies based on questionnaires should be interpreted with caution because of its subjective charac- 
ter, however, it appears that urologists have a seriously demanding workload for neck and shoulder region. 2, 6,7,10,12,15,16

Age and medical history, for example rheumatic disease, back hernia, neck hernia, and scoliosis, appeared to be significant risk factors for development of musculoskeletal complaints. Younger urologists appeared to be more at risk to develop musculoskeletal complaints than the older urologists. This could be explained by the fact that younger urologists are less experienced and endure more muscle tension during their learning curve for endourology and laparoscopy. This corresponds with the findings of Sari and coworkers. ${ }^{8}$

A recent study of Nieboer ${ }^{17}$ and associates found evidence that short term training of the non-dominant hand during 3 weeks resulted in a better performance of the dominant hand when performing simple laparoscopic tasks in a box-trainer, due to the "intermanual transfer of motor skills" phenomenon. Training of the non-dominant hand did not show a decrease in tension of the shoulder and neck muscles. ${ }^{18}$ Future studies are recommended to investigate if long-term training in a skills laboratory has a reducing effect on muscle tension, because, hypothetically, more experience in a certain skill result in less stress and muscle tension.

Szeto and colleagues" showed that $35.6 \%$ of the surgeons reported to "work through pain so that the quality of their work would not suffer". As a consequence, musculoskeletal complaints could result in chronic problems and loss of quality of care, quality of urologists' life and even to absence of work. The denial of pain should be taken seriously as sick absence attributed to musculoskeletal complaints does occur among urologists, which are confirmed by current study results. No firm evidence has been found in literature for the consequences of muscle fatigue in per- and post operative outcomes ${ }^{3}$, however, patient safety, urologists' quality of life and economic constraints due to an urologist who is not able to do surgery or due to sick absence should definitely be taken into consideration.

Preferably, we recommend methods to prevent these musculoskeletal problems. In the past decennia extensive ergonomic studies have been conducted to optimize instruments and to develop guidelines for laparoscopic surgery. ${ }^{19-22}$ Several studies have shown that instrument handles does not fit all hand sizes. Surgeons with smaller surgical glove size (5.5-6.5) needed hand treatment more often and experienced more musculoskeletal complaints compared to surgeons with a larger size. ${ }^{23-25}$ To narrow the gap between users and product designers, a variety of surgeons with different statures and features should be involved in the development process of endoscopic instruments.

Table height is defined as the distance from the tabletop to the floor. ${ }^{26}$ Berguer et al. ${ }^{27}$ recommended a table height of $5 \mathrm{~cm}$ below elbow height, with an acceptable range of $12,5 \mathrm{~cm}$ below to $2,5 \mathrm{~cm}$ above elbow height for open surgery. However, these recommendations are not sufficient for laparoscopy because of the length of the laparoscopic 
instruments, and because of the raise of the patients' abdomen from the pneumoperitoneum. The recommended optimum table height is when the instrument handles are at elbow height with a range of $10 \mathrm{~cm}$ below elbow height. ${ }^{27}$ This corresponds with a table height $64-77 \mathrm{~cm}$ above the floor.

Considering monitor placement it is suggested that monitors should be placed straight in front of the viewer in line with the forearm instrument, avoiding axial rotation of the neck and back. The head should be slightly flexed at an angle of $15-30^{\circ}$ downward..$^{22,26}$

The ideal body posture for a laparoscopic surgeon is standing in a neutral position, the arms are slightly abducted, retroverted and rotated inward at shoulder level. ${ }^{22,26}$ The elbows are bend at about $90-120^{\circ}$, the wrist are slightly extended and the hands are completely relaxed. ${ }^{28}$

The recommended monitor height and body posture, however, are assumed to be the same for endourological procedures as for laparoscopic procedures; ergonomic guidelines for endourological procedures are not yet established as it is for laparoscopic procedures.

Previous studies already acknowledged unawareness of ergonomic guidelines among surgeons (10) and our study results show only slightly better results. Surprisingly, almost $25 \%$ of the urologists claimed to have little knowledge about ergonomic rules and $8 \%$ of the urologists reported to have a total lack of knowledge considering ergonomics. Nevertheless, the majority of all respondents (89.9\%) were willing to improve ergonomic awareness, preferably by integrating ergonomics in hands on training. Hence, we have to search for strategies to collaborate ergonomists and urologists to transfer these knowledge and guidelines into urological practice. The authors therefore recommend introducing methods that increase ergonomic awareness among program directors to teach their residents in the operation room and to incorporate ergonomic rules into hands on training programs in skills laboratory.

In addition, robot-assisted surgery should be taken into account. This type of surgery offers significant advantages in terms of physical ergonomics for the surgeon who sits comfortably in front of the master console working with tool handles that offers more degrees of freedom, compared to conventional laparoscopic instruments. However, the robot amplifies range so there is no need for twisting hands, and stretching arms and shoulders in uncomfortable positions. ${ }^{29}$

A limitation of this study is that it relies on self-reported data instead of physical examination. To investigate the exact musculoskeletal complaints and whether these can be attributed to urological practice, long term follow up with measurement of muscle tension should be conducted in future studies.

Second limitation is that we did not include robot-assisted laparoscopy, hand-assisted laparoscopy, single-site laparoscopy and natural orifices translumenal endoscopic surgery, open surgery and residents. It would be interesting to investigate if the self- 
reported musculoskeletal complaints are solely due to laparoscopy and endourology or if other types of surgery also lead to these musculoskeletal complaints.

Differences between hospitals in each country can be found. ${ }^{6}$ Many operation rooms differ in their armamentarium for urological procedures. Examples are direct-view scopes or video assisted scopes for transurethral resection, movable or non-movable monitors and performing resections in standing or sitting position. In our study, this may have led to underreporting of physical complaints of urologists who still depend on non-ergonomic devices.

\section{CONCLUSION}

This study showed a high prevalence of musculoskeletal complaints among urologists who perform endourology and laparoscopy and a lack of knowledge about ergonomic rules. In concordance with our findings, we underline the importance to improve ergonomic awareness, and to involve ergonomists in the development of urological skills training to incorporate knowledge of surgeon' safety with patient safety in the early phase of urological practice.

\section{Acknowledgement}

The authors would like to thank all urologists who responded on the questionnaire. The authors would also like to thank Mereke Gorsira for her editorial assistance. 


\section{REFERENCES}

1. Luttmann A, Jager M and Sokeland J. Ergonomic assessment of the posture of surgeons performing endoscopic transurethral resections in urology. J Occup Med Toxicol. 2009; 4: 26.

2. Berguer R, Rab GT, Abu-Ghaida H, Alarcon A and Chung J. A comparison of surgeons' posture during laparoscopic and open surgical procedures. Surg Endosc. 1997; 11: 139-42.

3. Bagrodia A and Raman JD. Ergonomics considerations of radical prostatectomy: physician perspective of open, laparoscopic, and robot-assisted techniques. J Endourol. 2009; 23: 627-33.

4. Berguer R, Smith WD and Chung YH. Performing laparoscopic surgery is significantly more stressful for the surgeon than open surgery. Surg Endosc. 2001; 15: 1204-7.

5. Gofrit ON, Mikahail AA, Zorn KC, Zagaja GP, Steinberg GD and Shalhav AL. Surgeons' perceptions and injuries during and after urologic laparoscopic surgery. Urology. 2008; 71: 404-7.

6. Reyes DA, Tang B and Cuschieri A. Minimal access surgery (MAS)-related surgeon morbidity syndromes. Surg Endosc. 2006; 20: 1-13.

7. Szeto GP, Ho P, Ting AC, Poon JT, Cheng SW and Tsang RC. Work-related musculoskeletal symptoms in surgeons. J Occup Rehabil. 2009; 19: 175-84.

8. Sari V, Nieboer TE, Vierhout ME, Stegeman DF and Kluivers KB. The operation room as a hostile environment for surgeons: physical complaints during and after laparoscopy. Minim Invasive Ther Allied Technol. 2010; 19: 105-9.

9. Berguer R, Forkey DL and Smith WD. Ergonomic problems associated with laparoscopic surgery. Surg Endosc. 1999; 13: 466-8.

10. Wauben LS, van Veelen MA, Gossot D and Goossens RH. Application of ergonomic guidelines during minimally invasive surgery: a questionnaire survey of 284 surgeons. Surg Endosc. 2006; 20: 1268-74.

11. Widanarko B, Legg $S$, Stevenson $M$, et al. Prevalence and work-related risk factors for reduced activities and absenteeism due to low back symptoms. Appl Ergon. 2012; 43: 727-37.

12. Luttmann A. Working conditions, muscular activity and complaints of office workers International Journal of Indstrustrial Ergonomics. 2011; 404: 10.

13. Healy KA, Pak RW, Cleary RC, Colon-Herdman A and Bagley DH. Hand problems among endourologists. J Endourol. 2011; 25: 1915-20.

14. Elkoushy MA and Andonian S. Prevalence of orthopedic complaints among endourologists and their compliance with radiation safety measures. J Endourol. 2011; 25: 1609-13.

15. Park $A$, Lee $G$, Seagull FJ, Meenaghan $N$ and Dexter D. Patients benefit while surgeons suffer: an impending epidemic. J Am Coll Surg. 2010; 210: 306-13.

16. Kaya Ol, Moran M, Ozkardes AB, Taskin EY, Seker GE and Ozmen MM. Ergonomic problems encountered by the surgical team during video endoscopic surgery. Surg Laparosc Endosc Percutan Tech. 2008; 18: 40-4.

17. Nieboer TE, Massa M, Weinans MJ, Vierhout ME, Kluivers KB and Stegeman DF. Does Training of the Nondominant Upper Extremity Reduce the Surgeon's Muscular Strain During Laparoscopy? Results From a Randomized Controlled Trial. Surg Innov. 2012.

18. Nieboer TE, Sari V, Kluivers KB, Weinans MJ, Vierhout ME and Stegeman DF. A randomized trial of training the non-dominant upper extremity to enhance laparoscopic performance. Minim Invasive Ther Allied Technol. 2012; 21: 259-64.

19. Manasnayakorn S, Cuschieri A and Hanna GB. Ergonomic assessment of optimum operating table height for hand-assisted laparoscopic surgery. Surg Endosc. 2009; 23: 783-9. 
20. Manasnayakorn S, Cuschieri A and Hanna GB. Ideal manipulation angle and instrument length in hand-assisted laparoscopic surgery. Surg Endosc. 2008; 22: 924-9.

21. van Det MJ, Meijerink WJ, Hoff C, van Veelen MA and Pierie JP. Ergonomic assessment of neck posture in the minimally invasive surgery suite during laparoscopic cholecystectomy. Surg Endosc. 2008; 22: 2421-7.

22. van Det MJ, Meijerink WJ, Hoff C, Totte ER and Pierie JP. Optimal ergonomics for laparoscopic surgery in minimally invasive surgery suites: a review and guidelines. Surg Endosc. 2009; 23: 1279-85.

23. Adams DM, Fenton SJ, Schirmer BD, Mahvi DM, Horvath $\mathrm{K}$ and Nichol P. One size does not fit all: current disposable laparoscopic devices do not fit the needs of female laparoscopic surgeons. Surg Endosc. 2008; 22: 2310-3.

24. Berguer $\mathrm{R}$ and Hreljac $A$. The relationship between hand size and difficulty using surgical instruments: a survey of 726 laparoscopic surgeons. Surg Endosc. 2014; 28:1051-5.

25. Sutton E, Irvin M, Zeigler C, Lee G and Park A. The ergonomics of women in surgery. Surg Endosc. 2014; 28:1051-5.

26. Wauben LSA, A., Goossens R.H. Ergonomics in the operating room-an overview. Ergonomics: Design, Integration and Implementation. New York: Nova Publishers, 2009.

27. Berguer R, Smith WD and Davis S. An ergonomic study of the optimum operating table height for laparoscopic surgery. Surg Endosc. 2002; 16: 416-21.

28. Berguer R, Forkey DL and Smith WD. The effect of laparoscopic instrument working angle on surgeons' upper extremity workload. Surg Endosc. 2001; 15: 1027-9.

29. Stylopoulos N and Rattner D. Robotics and ergonomics. Surg Clin North Am. 2003; 83: 1321-37. 




\section{PURPOSE OF THIS THESIS}

Simulator based training has the potential to supplement learning in clinical practice in order to decrease the initial stages of the learning curve in an environment that does not compromise patient safety. Nowadays simulator based training has been widely accepted as a supplement to learning in clinical practice. However it has not yet established its position in current urological curriculum. In this thesis we investigated the main research question: "How can simulator based training and assessment be developed to improve learning in urology and to narrow the gap between research and integration in clinical practice?"

\section{CONCLUSIONS}

In this thesis we described the development and evaluation of a modified basic laparoscopic skills program to urological purposes; the Program for Laparoscopic Urological Skills (PLUS). For the Dutch setting the PLUS was valued positively for training basic laparoscopic skills by urologists, urological residents and novices. Furthermore, we found a strong correlation between laparoscopic experience and task performance, thereby confirming its face, content and construct validity.

Subsequently we aimed to promote the standardization of basic laparoscopic skills assessment. Therefore we investigated the reliability of the PLUS as assessment tool and established a certification standard for second-year urological residents based on the generalized examinee-centred method. The results of this study showed that the PLUS is a reliable assessment tool for credentialing purposes, with two judges and two trials being sufficient to reach good reliability. The PLUS assessment offers quality and time criteria for the completion of basic laparoscopic tasks and $63 \%$ of the second-year residents in the Netherlands passed the PLUS assessment when certification standard was set on the novice/intermediate boundary. We concluded that the PLUS assessment offers a justifiable starting point from which residents can be allowed to continue their development towards the next level in the laparoscopic curriculum.

Evaluating the basic laparoscopic skills of European residents in their final year of residency, we discovered that only $4.3 \%$ of them meet the qualification standards to pass the PLUS assessment. Although quality of performance is good, most residents did not pass the validated time criteria of the examination. We therefore conclude that the timing and setting of the examination should be carefully evaluated to determine its future use. Moreover, regular laparoscopic training or a dedicated fellowship in laparoscopy should improve the laparoscopic level of residents in urology who intend to perform laparoscopy 
This thesis also addresses the validation process of the TURPsim ${ }^{\mathrm{TM}}$ to train transurethral resection of the prostate (TURP), according to a curriculum-based approach. In this study the outcome parameters of the validation process were not solely based on performance metrics given by the simulator, but we established them trough a training needs analysis (TNA). It appeared that isolated simulator based training did not cover all procedural steps and pitfalls of the TURP procedure. However, investigation of the face, content and construct validity of the TURPsim ${ }^{\mathrm{TM}}$ showed positive results, suggesting that the use of this simulator is recommended in the current urological curriculum to train basic and procedural TURP skills.

TNA appeared to be of paramount importance in the development and evaluation process to determine procedural steps and to identify pitfalls. The study in chapter 6 described how training needs analysis for the nephrostomy procedure could be structured by integrating the 4C/ID model and cognitive task analysis. This model aimed to identify automatic and non-automatic procedural steps and its pitfalls. The final result of the study was a blueprint containing whole task practices with increasing difficulty. In addition, it indicates part task practices for certain skills that require a high level of automaticity. Continuation of training needs analysis for different urological procedures may be helpful in moving forward the development of training programs and to identify the place of simulators in the current curriculum.

Finally we focused on human factors of minimal invasive urological skills. High prevalence of experienced musculoskeletal complaints was found among urologists predominantly related to endourology and laparoscopy. Majority of the respondents indicate to have little knowledge about ergonomics in the operation room. Hence, we suggest integration of ergonomics in training programs preferably early in the residency curriculum to gain knowledge and awareness and hopefully to offer possibilities to prevent these complaints in the future.

\section{GENERAL DISCUSION}

\section{Development of Training}

The aim of education in urology is quality assurance at all levels. Ultimate objective is to ensure and improve patient care, but the influence of educational research on simulator based training in an existing curriculum has been limited by a lack of scientific methodology and inefficient provision of resources. In this thesis we described a methodology to unravel experts' way of performance and thinking (chapter 5 and 6). First is to identify procedural steps and second all steps should be defined as automated or non-automated. A context of required knowledge before starting skills training is prerequisite. 
The automatization process involves learning to recognize important environmental cues that signal what technical and non-technical steps need to be applied to achieve a certain goal. Through practice, these associations and steps increase reliability and speed of performance. Over time mental effort needed to perform that skill will decrease. ${ }^{1}$ Ericson who stated that deliberate practice is crucial to attain expert level also underlines this. Nonetheless, with merely repetitive training improvement of performance will arrest at a certain level. ${ }^{2,3}$ Having this knowledge, in the ideal situation urologists would develop their own simulator with endless opportunities for deliberate practice involving part tasks and whole tasks with increasing complexity at no costs before performing on real patients. In reality we have to cope with financial and time constraints. This raises the question; "which urological skills require a high level of automaticity that need to be trained on a simulator to attain and maintain expert level?"

However, in this context simulator based training is only supplemental to clinical practice, since case variation and experience needed for "non-automatic" skills proficiency will be learned with direct patient care. This is also underlined by the theory of Rasmussen that differentiates human behaviour into three levels (table 1): skills, rules and knowledge. ${ }^{4}$ By shifting training of skills that require high automaticity from the clinical setting to skill laboratory, "skill based behaviour" and even "rule based behaviour" could be attained without constraining patient safety. Subsequently knowledge based behaviour to learn non-automatic skills can be enhanced in clinical practice or in a stepwise training program were simple simulators are combined with more complex training modalities before transfer to clinical practise is made. A blueprint like one from the 4C/ID model could be helpful in the development of a training program. In our opinion collaboration with urologists, engineers and educationalists, in a preliminary phase of the development process is inevitable.

Table 1: Rasmussen' theory of human behaviour

\begin{tabular}{ll}
\hline Skill based behaviour & $\begin{array}{l}\text { Task performance is highly automated and takes place without } \\
\text { conscious control } \\
\text { Rule based behaviour }\end{array}$ \\
Knowledge based behaviour & $\begin{array}{l}\text { Stored rules and procedures, for example: knowledge of anatomy, } \\
\text { pathofysiology and procedural steps }\end{array}$ \\
& $\begin{array}{l}\text { This knowledge is on the forefront when unexpected, unfamiliar } \\
\text { situations occur. These cannot be automated and strategy to overcome } \\
\text { these situations are based on mental processes, for example experience }\end{array}$ \\
\hline
\end{tabular}

The studies in chapter 2,3 and 4 described the development of a training program and assessment for basic laparoscopic skills. We also measured proficiency levels of residents in different stages of their urological career. We expected that residents would be on a continuous pathway of development, with better scores when reaching the end of 
their residency. ${ }^{5}$ However it appears that last year residents perform even worse on basic laparoscopic skills than second year residents in the Netherlands. This raises the question; "what is the reason that only few last year residents pass the PLUS exam, whilst the proficiency standard was set on the very low boundary of novice/intermediate level?"

There are various possible explanations. First we measured European residents in their last year. It is likely that major differences exist between urological residency curricula of different European countries. Heterogeneity of the cohort could have influenced the results. A study of Menhadji et al tracked performance scores of urological residents over four years for open, laparoscopic and robotic skills. ${ }^{6}$ Their study results showed improvement in all 3 types of surgery, however, the lowest performance scores and lowest improvement scores over years were consistently demonstrated for laparoscopic skills. This study illustrates the complexity of learning and maintaining laparoscopic skills, underlining the necessity for continuous training to secure automaticity of basic laparoscopic skills.

Second, we did not find evidence for predicting parameters for passing scores on the PLUS assessment. However, results from the questionnaires showed that most residents did not train nor were exposed to real live laparoscopic operations in the past four weeks prior to the examination. Obviously, simulator based training of laparoscopic skills is not yet established in daily practice of the current urological residency curricula in Europe. The difficulty with implementation of simulator based skills training in an existing curriculum is the change in organizational programs and the willingness of clinicians to adapt. Our results illustrates that proving validity of a training program only is not enough to improve ones' skills.

\section{Development of Assessment}

Changes in clinical practice, medical education and, urgent calls from government and the public stress the need to develop and maintain urological skills performance on a high level. Increasing emphasis on competency-based rather than volume-based assessment is recognized in recent literature. ${ }^{7}$ In the study we described in chapter 3 we aimed to set proficiency standards for basic laparoscopic skills offering time and quality criteria. Most challenging in determining proficiency standards is to reduce the arbitrariness that is coherent with any form of setting a cut-off point. Crucial in this process is the reasoning why the assessment should be developed and what purpose it needs to serve. We chose to set the proficiency standard for basic laparoscopic skills of second year residents in urology on the boundary between novices and intermediates to filter out the truly incompetent examinees. We believe that such a standard is justifiable as a starting point for residents' further competency development towards the intermediate and expert levels during the next four years of their residency. Therefore, the PLUS as- 
sessment cannot be interpreted as proof of competence in procedural skills, but only as proof of meeting the very minimum standard of basic technical proficiency.

The qualification and certification of urological laparoscopic skills are still in a preliminary phase not to mention other urological procedures like open cystectomy, laparoscopic prostatectomy, percutaneous nephrolithotomy, transurethral resection of the prostate etcetera, for which no proficiency standards are established. It is known from literature that competency based rather than volume based assessment is crucial. The "levels of expertise" described by Dreyfus\&Dreyfus (1986) could serve as a conceptual model to define outcome parameters for each level, thus establishing proficiency standard for each plateau in the learning curve. This framework outlined the stages of progression from novice to expert where knowledge and skills move from basics with little situational perception in novices to the holistic intuitive approach of experts. ${ }^{5}$

Additional key aspects for development of a competency based assessment are defining the skills to be automatic or non-automatic, who needs to be assessed, when should be assessed and finally determine what assessment format, summative or formative, ${ }^{5}$ is most appropriate for qualifying that particular skill. When putting all frameworks together it becomes apparent that when assessing learners one should take into account those factors mentioned above, preferably integrating knowledge (cognition), psychomotor skills and level of competence.

\section{Human factors}

Laparoscopy is considered more difficult to perform compared to open surgery. There are less degrees of freedom, there is less tactile feedback, the urologists have to cope with instruments that move contrary to the hand (fulcrum effect) and 2-dimensional screen view has to become 3-dimensional view in the surgeons mind. Since the late nineties industrial designers were aware of developing laparoscopic instruments that must be efficient and comfortable for the users and ergonomic guidelines were developed, but implementation of those guidelines hampered. ${ }^{8-12}$ The results from the study described in chapter 7 showed that $62 \%$ of the urologists had musculoskeletal complaints that were considered work related according to their opinions. It appeared that laparoscopy and endourology were significant risk factors.

However $25 \%$ of the respondents had little knowledge about the ergonomic guidelines for table height, monitor height and body posture. Implementation strategies to increase awareness of the guidelines should be evaluated and in our opinion awareness should start at the very beginning of ones' laparoscopic career. For example, course directors and proctors should integrate the ergonomic aspect of laparoscopy and endourology within their skills trainings. With the ongoing improvement and development of new techniques and instruments it is of paramount importance that further collabora- 
tion between urologists and instrument designers should be continued. General goal is to reduce physical discomfort and injuries for urologists and improve patients' safety.

\section{VALORISATION: PRACTICAL IMPLICATIONS AND FUTURE PERSPECTIVES}

In this thesis we tried to answer the main question how simulator based training and assessment can be developed to improve learning in urology and to narrow the gap between research and integration in clinical practice. We therefore validated two simulators, using training needs analysis, and we investigated the most justifiable proficiency standard for second year urology residents. However, main limitation throughout this thesis is the limited numbers of urologists and residents in the Netherlands. For studies to validate simulators and more important for setting proficiency standards large numbers of participants are preferable. In future studies international collaboration should attempt to gain numbers of participants, with documented levels of experience and training. Consequently study results will be applicable not only for the Dutch urology setting but for a wider urological field. First steps of international collaboration were made in the study described in chapter 4.

Learning complex urological tasks is not only a matter of motor skills performance, but comprises mental actions in combination with physical movements. Awareness of cognition, or non-technical skills, and technical skills as variables of competency is beginning to appear in medical education. ${ }^{13,14}$ However, many studies of learning curves or urological proficiency do not assess competency in such a holistic way. In chapter 6 we investigated a conceptual model to unravel experts knowledge, thought processes and goal structures and to make the gathered information applicable by presenting a blueprint according to the 4C/ID model. Limitation is that we only used semi-structured interview as a method of cognitive training analysis.

There are various other methods like observation studies or Delphi method to unravel experts' knowledge. Future studies should focus on different methods to investigate what is most time efficient for cognitive training analysis aimed to indentify training needs. Furthermore future studies are needed to investigate whether simulator based training according to the 4 C/ID design is an effective tool to reduce the initial stages of the learning curve.

In this thesis we only investigated training needs and simulator based training for the basic competency levels for laparoscopy, transurethral resection of the prostate and the nephrostomy procedure. The content of training, the method of training in and outside the operation theatre and the methods of assessment for different urological proce- 
dures and preferably for the different plateaus in the learning curves for each procedure remained to be researched.

\section{Collaboration}

Development of simulators and advances in new techniques in urology are ongoing processes. It becomes clear that development of simulator based training and assessment is not only a matter of validation of a single simulator. Knowledge and insights from other domains beyond urology like engineers, educationalists, ergonomist, instructional designers and implementation experts, are of paramount importance to bridge the gap between simulator based research and clinical practice. Most challenging in this process is to understand each others terminology.

Theories from instructional design to identify training needs and to create a blueprint according to experts' knowledge could bridge the gap to simulator engineers who are responsible for the creation of a simulator with an inbuilt educational program. To use a simulator in a training program educationalists and ergonomists can contribute in the development processes what, when and where should be trained outside the operation room in the curriculum to reduce the learning curve before transferred to clinical practice. Awareness of human factors is under-exposed in current urological practice. Therefore we recommend collaboration with ergonomists early in the curriculum, to integrate ergonomics in the early training programs of residents. Finally urologists and educationalist both have a major role in defining proficiency standards and what type of assessment is most appropriate to measure a particular skill.

\section{Curriculum based training}

Although effectiveness and validity of simulator based training was demonstrated, adoption of these new insights in urological residency programs is still marginal. ${ }^{15,16}$

Various studies have shown, that the adoption of useful research findings in day-to-day clinical practice tends to be slow and difficult due to a range of factors like lack of time, financial constraints, lack of resources necessary changing daily routines etcetera. ${ }^{17,18}$ Future studies should focus on current urological residency programs from the perspective of organization design. Taken that as a starting point, implementation strategies should be researched on how to transfer simulator based skill training into an existing urological curriculum, without constraining learning opportunities in clinical practice.

Reasonable outcome parameters and accessory proficiency standards should be defined for each level of expertise, described by the theory of Dreyfus\&Dreyfus. ${ }^{5}$ Consequently outcome parameters will vary from automaticity of a sub-skill, procedural outcome in terms of blood loss for example, to patient outcome in terms of oncological free margins for example and even more. The integrated methodology of 4C/ID model and CTA could be useful tools for analysis. After defining relevant outcome parameters 
and implementation of the simulator based skill training cost-effectiveness of the new curriculum should be investigated, whilst educational research continues to improve learning in urology and to ensure patient safety. 


\section{REFERENCES}

1. Clark RE, Feldon DF, van Merrienboer JJ, Yates KA and Early S. Cognitive Task Analysis. In: al SJMe, (ed.). Handbook of Research on Educational Communications and Technology. London: Taylor and Francis Group, 2008, p. 577-93.

2. Ericsson KA. Deliberate practice and the acquisition and maintenance of expert performance in medicine and related domains. Acad Med. 2004; 79: S70-81.

3. Ericsson KA. Deliberate practice and acquisition of expert performance: a general overview. Acad Emerg Med. 2008; 15: 988-94.

4. Wentink M, Stassen LP, Alwayn I, Hosman RJ and Stassen HG. Rasmussen's model of human behavior in laparoscopy training. Surg Endosc. 2003; 17: 1241-6.

5. Dornan T, Mann K, Scherpbier AJ and Spencer J. Medical Education: Theory and Practice. London: Churchill Livingstone Elsevier, 2011.

6. Menhadji A, Abdelshehid C, Osann K, et al. Tracking and assessment of technical skills acquisition among urology residents for open, laparoscopic, and robotic skills over 4 years: is there a trend? J Endourol. 2013; 27: 783-9.

7. Gohil R, Khan RS, Ahmed K, et al. Urology training: past, present and future. BJU Int. 2012; 109: 1444-8.

8. Berguer R. Surgical technology and the ergonomics of laparoscopic instruments. Surg Endosc. 1998; 12: 458-62.

9. Berguer R. Surgery and ergonomics. Arch Surg. 1999; 134: 1011-6.

10. Adams DM, Fenton SJ, Schirmer BD, Mahvi DM, Horvath $\mathrm{K}$ and Nichol P. One size does not fit all: current disposable laparoscopic devices do not fit the needs of female laparoscopic surgeons. Surg Endosc. 2008; 22: 2310-3.

11. Berguer R, Forkey DL and Smith WD. The effect of laparoscopic instrument working angle on surgeons' upper extremity workload. Surg Endosc. 2001; 15: 1027-9.

12. Berquer R, Smith WD and Davis S. An ergonomic study of the optimum operating table height for laparoscopic surgery. Surg Endosc. 2002; 16: 416-21.

13. Khan N, Abboudi H, Khan MS, Dasgupta P and Ahmed K. Measuring the surgical 'learning curve': methods, variables and competency. BJU Int. 2013.

14. van Merrienboer JJ and Sweller J. Cognitive load theory in health professional education: design principles and strategies. Med Educ. 2010; 44: 85-93.

15. Ahmed K, Jawad M, Abboudi M, et al. Effectiveness of procedural simulation in urology: a systematic review. J Urol. 2011; 186: 26-34.

16. Schout BM, Hendrikx AJ, Scheele F, Bemelmans BL and Scherpbier AJ. Validation and implementation of surgical simulators: a critical review of present, past, and future. Surg Endosc. 2010; 24: 536-46.

17. Grol R and Grimshaw J. Evidence-based implementation of evidence-based medicine. Jt Comm J Qual Improv. 1999; 25: 503-13.

18. Grol R and Wensing M. What drives change? Barriers to and incentives for achieving evidencebased practice. Med J Aust. 2004; 180: S57-60. 



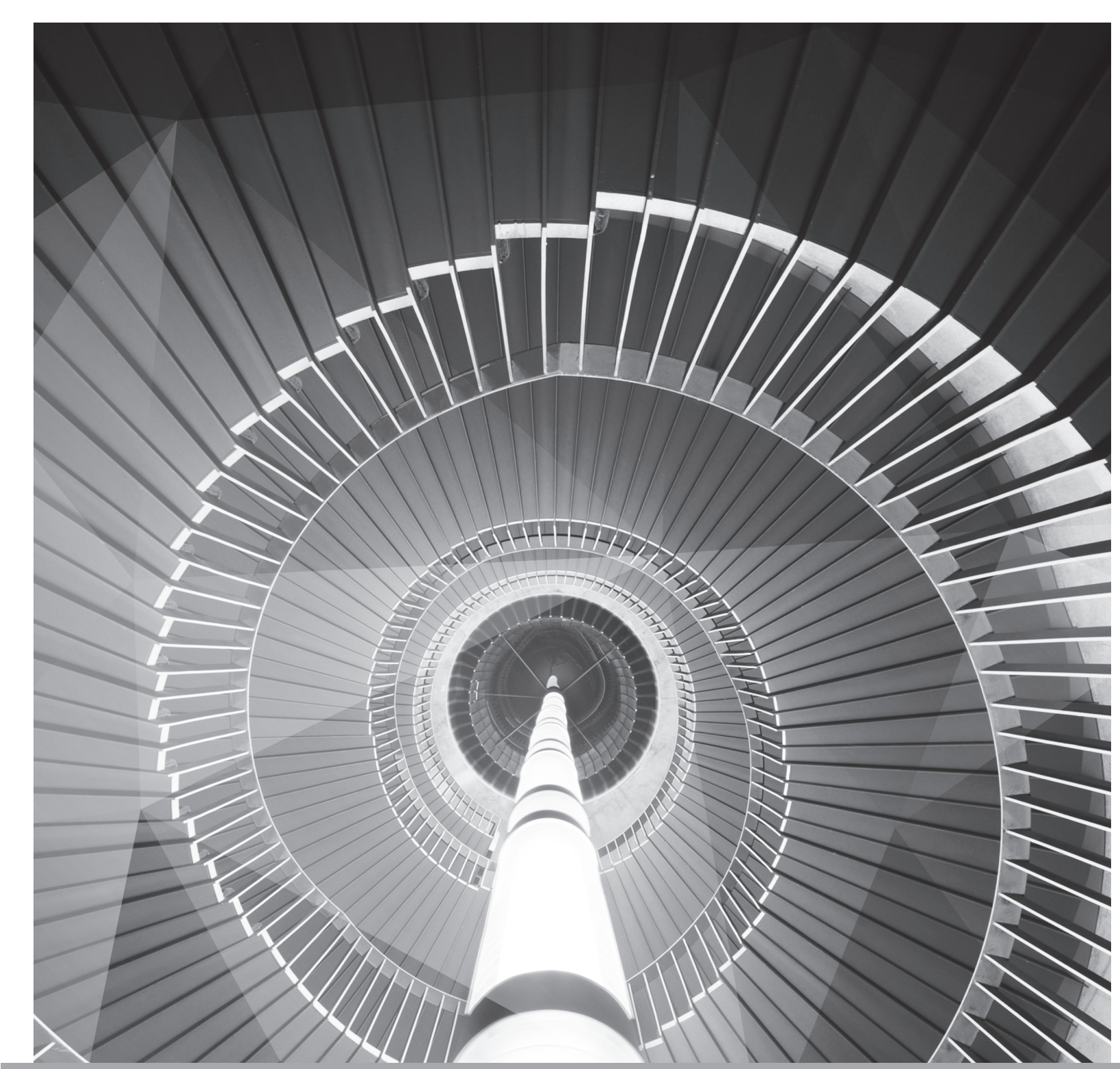

\section{Summary}





\section{SUMMARY}

Minimal invasive procedures, like laparoscopy and endourology, are common practice in urology. Laparoscopy is a video assisted procedure using instruments that enters the patients' body via small incisions of approximately $1 \mathrm{~cm}$. In endourological procedures instruments enter the patients' body via natural orifices, for example the urethra. All types of minimal invasive urology have a longer learning curve compared to open surgery as a result of different instruments, less tactile feedback and limited workspace. ${ }^{1-4}$

Traditionally learning surgical skills is based on the paradigm of the Halstedian model, "see one, do one, teach one".5-8 However, at present-day, the European working time directive (2004), ethical and financial considerations raise barriers to achieving appropriate levels of mastery through training in the operating theatre alone. ${ }^{9-11}$ Furthermore, performing a first procedure directly on a patient is inacceptable as legal considerations are making high demands on surgeons' proficiency and standards of patient safety., ${ }^{5,12}$ Educationalists are therefore searching for other methods to complement the traditional way to learn and assess surgical skills, such as simulator based training.

Simulator based training has been advocated as an adjunct to learning in clinical practice. Key advantage of simulator based training is that it allows learners to gain procedural skills in a safe setting outside the clinical area where they can make mistakes without doing harm to the patient.6,7,14

Before a simulator can be implemented in a curriculum its effectiveness should be established. In other words, the simulator needs to train what it is intended to train. Effectiveness of a simulator can be researched by a systematic validation process. ${ }^{15}$

Urgent-calls from the government and the public necessitate well-defined proficiency standards to safeguard the quality of care. Traditionally proficiency in surgery has been verified by case-volume. However, the use of case-numbers to assess surgical competence is far from objective to evaluate technical skills. A high number of cases, does not automatically mean that those cases were performed well, with appropriate patient outcomes. ${ }^{8,16}$ One of the challenges of developing proficiency based assessment in urology is how to establish pass-fail standards for the performance of skills.

Subsequently, it is important to identify what role the simulator can fulfil to train which part of the procedure and how the simulator based training can be integrated in the curriculum. Therefore a "training needs analysis" should be conducted to specify training objectives. Urological skills can be considered as complex tasks, including psychomotor and cognitive decision-making skills. From the perspective of instructional design complex tasks are defined as those for which their performance require the integrated use of non-automated and automated knowledge and skills. ${ }^{17}$ Most difficult in analysis of training needs is filtering out expert experience to identify succeeding procedural steps and decision-making processes, and define for which part of the procedure simu- 
lator based training is most appropriate. Strategies from the field of instructional design could be useful to structure the training needs analysis for urological training programs.

Minimal invasive urology confronts urologists not only with a longer learning curve, urologists also endures ergonomic challenges because they have to stand in a static position for a long time. The science of human factors, also called ergonomics, analyses these challenges and formulate guidelines for creating a work environment that is safe for the surgeon and the operating team

In this theses we focused on the following general research question:

How can simulator based training and assessment be developed to improve learning in urology and to narrow the gap between research and integration in clinical practice? (Chapter 1).

In chapter $\mathbf{2}$ we described the development and evaluation of a modified basic laparoscopic skills program to urological purposes; the Program for Laparoscopic Urological Skills (PLUS). The PLUS consisted of five basic tasks; three tasks were abstracted from the Fundamentals of Laparoscopic Surgery program, and two additional tasks were developed under continuous evaluation of expert urologist. Fifty participants with different experience levels performed all 5 tasks twice in succession. Afterwards they had to fill out a questionnaire concerning usefulness and realism of the simulator. For the Dutch setting the PLUS was valued positively for training basic laparoscopic skills by urologists, urological residents and novices. The usefulness of the PLUS was rated very high; 4.55 on a scale from 1 (=not useful) to 5 (=useful). Furthermore, we found a strong correlation between laparoscopic experience and performance time. The decrease in time for experts versus novices ranged from $46 \%$ to $74 \%$ for all five tasks. Also substantial correlations were found between experience and quality ratings; and the number of dropped objects. A relative increase of $18 \%$ in quality ratings was found for experts versus novices. Apparently the PLUS is judged to be a useful simulator for the urological curriculum. In addition the results show that the PLUS discriminates between different experience levels in laparoscopy and that it has substantial learning effect especially for trainees that are in the beginning of their learning curve.

The study described in chapter 3 aimed to promote the standardization of basic laparoscopic skills assessment. Therefore we investigated the reliability of the PLUS as assessment tool and established a certification standard for second-year urological residents based on the generalized examinee-centered method. This educational approach uses the linear relationship between assessment scores and degree of procedural experience of multiple reference groups. Assessment-scores on the boundaries between different categories or groups are then used as pass/fail criteria. In this study 50 participants with different levels of experience were assessed on performance time and performance 
quality to investigate the reliability of the PLUS assessment. Generalizability coefficient of 0.8 , on a scale of 1 , is considered to indicate good reliability for assessment purposes. Pass/fail standards were based on laparoscopic experience: novices, intermediates (1-100 laparoscopic procedures), and experts (>100 laparoscopic procedures). The pass/fail standards were investigated for the PLUS performances of 33 second-year urological residents.

The results of this study showed that the PLUS is a reliable assessment tool for credentialing purposes, with two judges and two trials being sufficient to reach good reliability. Pass/fail scores were determined for the novice/intermediate boundary and the intermediate/expert boundary. Pass rates for second-year residents were $63.64 \%$ and $9.09 \%$, respectively. The PLUS assessment offers quality and time criteria for the completion of basic laparoscopic tasks and $63 \%$ of the second-year residents in the Netherlands passed the PLUS assessment when certification standard was set on the novice/intermediate boundary. We concluded that the PLUS assessment with the pass/ fail criteria set on the novice/intermediate boundary, offers a defensible starting point from which residents can be allowed to continue their development towards the next level in the laparoscopic curriculum.

We were then interested in the basic laparoscopic skills level of European residents and if they would pass the validated criteria according to the PLUS assessment on the novice/intermediate boundary. Chapter 4 described a study where European-Basic Laparoscopic Urological Skills (E-BLUS) performances of 70 last year urology residents from different countries were analysed. The E-BLUS comprises the same five tasks and performance criteria as the PLUS. The results showed that only $4.2 \%$ of the last-year European residents meet the qualification standards to pass the PLUS assessment. Although quality of performance is sufficient (pass rate $=63 \%$ ) most residents did not pass the validated time criteria of the examination. Sixty-one percent of the examinees stated that they had no opportunity to train laparoscopic skills in the four weeks prior to the examination. We therefore conclude that the timing and setting of the examination should be carefully evaluated to determine its future use. Moreover, regular laparoscopic training or a dedicated fellowship in laparoscopy should improve the laparoscopic level of residents in urology.

The results of a validation study of a virtual reality simulator to train transurethral resection of the prostate (TURP), according to a curriculum-based approach are outlined in chapter 5.

In this study the outcome parameters of the validation process were not solely based on performance metrics given by the simulator, but we established them trough a training needs analysis (TNA). An expert panel to identify procedural steps and pitfalls conducted the TNA. Performance metrics of the simulator were compared with the TNA results. It appeared that 10 out of 22 procedural steps, resulted form the TNA, and 4 
out of 11 pitfalls were covered by the TURPsim ${ }^{\text {TM }}$. Subsequently, 22 novices (medical students), 22 intermediates (residents and urologists who performed 1 -50 complete TURP procedures) and 22 experts (urologists who performed $>50$ complete TURP procedures) followed a standardised training and performed 2 complete TURP procedures in succession. Afterwards they had to fill out a questionnaire regarding its usefulness and realism. Results showed that $93 \%$ of all participants qualified the TURPsim ${ }^{\mathrm{TM}}$ as a useful training model. Median general judgement about its usefulness was rated high; 7.3 on a scale of 1 (=not useful) to 10 (=very useful). Intermediates and experts had a significant faster resection time and less blood loss compared to novices. Despite the standardised training session prior to performance of a whole TURP procedure on the simulator, novices needed to re-resected previous lobes and they also resected the prostate in the incorrect order more frequently compared to intermediates and experts. A reason for this finding could be that novices received too much new information in a short amount of time, and for experts part of that information is already automatic. Also, experts appeared to resect more often with non-optimal visibility compared to novices. This could be explained by the fact that experts know from their experience were to resect safely. Our results showed that isolated simulator-based training did not cover all procedural steps and pitfalls of the TURP procedure according to the results of the TNA. In addition, the simulator does not contain metrics to differentiate between automated en non-automated skills. However, the TURPsim ${ }^{\mathrm{TM}}$ received positive judgements on realism and usefulness for the urological curriculum. Moreover it showed the ability to discriminate between trainees with different experience levels, suggesting that the use of this simulator is to be recommended in the current urological curriculum to train basic and procedural TURP skills.

TNA appeared to be of paramount importance in the development and evaluation process to determine procedural steps and to identify pitfalls. The study in chapter $\mathbf{6}$ described how training needs analysis for the nephrostomy procedure could be structured by integrating the 4C/ID model and cognitive task analysis. Aim of the nephrostomy procedure is to gain direct access from the skin to the renal system for urinary drainage, in case of an obstruction between the kidney and the bladder for example. The 4C/IDmodel aimed to identify automatic and non-automatic procedural steps and its pitfalls. Cognitive task analysis refers to a variety of interview and observation methods aimed at unravelling experts' performance of complex tasks to capture the knowledge, decision making processes and goal structures the experts rely on during task performances. In this study four urologists were asked to complete a questionnaire regarding the procedural steps, and eight expert urologists from seven different hospitals were invited to participate in a semi-structured group interview conducted by an educational expert, a researcher and an expert urologist. Aim of the semi-structured interview was to identify all steps and sub-steps of the procedure and define each (sub)-step as automatic or 
non-automatic. In addition they were asked to mention scenarios with different levels of difficulty.

The final result of the study was a blueprint containing whole task practices with increasing difficulty. In addition, it indicates part task practices for certain skills that require a high level of automaticity. Continuation of training needs analysis for different urological procedures may be helpful in moving forward the development of training programs and to identify the place of simulators in the current curriculum.

In chapter 7, we focused on human factors of minimal invasive urological skills. An online and hard copy version questionnaire was administered to urologists from different countries, performing endourology and laparoscopy. Of the 285 respondents, 245 $(86.0 \%)$ urologists experienced musculoskeletal complaints in the past 12 months and $62.1 \%$ were considered to be work related. An increase of age was associated with a decrease of the risk for complaints. Most common areas for chronic complaints were neck, back and shoulders. Almost $50 \%$ of the urologists experienced chronic musculoskeletal complaints, for which endourology and laparoscopy were significant risk factors. One third of the respondents indicated to have little knowledge about ergonomics in the operation room. Hence, we suggest integration of ergonomics in training programs preferably early in the residency curriculum to gain knowledge and awareness and hopefully to offer possibilities to prevent these complaints in the future.

In chapter $\mathbf{8}$ the general conclusion with regard to the main research question of this thesis is described, followed by a general discussion on development of simulator based training and assessment. In addition implications of ergonomic discomfort experienced by urologists are discussed. Finally, practical implications and suggestions for further research are presented. 


\section{REFERENCES}

1. Kumar U and Gill IS. Learning curve in human laparoscopic surgery. Curr Urol Rep. 2006; 7: 120-4.

2. Capelouto CC and Kavoussi LR. Complications of laparoscopic surgery. Urology. 1993; 42: 2-12.

3. Kerbl K and Clayman RV. Advances in laparoscopic renal and ureteral surgery. Eur Urol. 1994; 25: $1-6$.

4. Gallagher AG, McClure N, McGuigan J, Ritchie K and Sheehy NP. An ergonomic analysis of the fulcrum effect in the acquisition of endoscopic skills. Endoscopy. 1998; 30: 617-20.

5. Kohn LT, Corrigan JM and Donaldson MS. To err is human: Building a safer health system. 7 ed. Washington DC: National Academy Press, 2008.

6. Dornan T, Mann K, Scherpbier AJ and Spencer J. Medical Education: Theory and Practice. London: Churchill Livingstone Elsevier, 2011.

7. Rodriguez-Paz JM, Kennedy M, Salas E, et al. Beyond "see one, do one, teach one": toward a different training paradigm. Qual Saf Health Care. 2009; 18: 63-8.

8. Reznick RK and MacRae H. Teaching surgical skills_changes in the wind. N Engl J Med. 2006; 355: 2664-9.

9. Kelly BD, Curtin PD and Corcoran M. The effects of the European Working Time Directive on surgical training: the basic surgical trainee's perspective. Ir J Med Sci. 2011; 180: 435-7.

10. Scott DJ. Patient safety, competency, and the future of surgical simulation. Simul Healthc. 2006; 1: 164-70.

11. Parsons BA, Blencowe NS, Hollowood AD and Grant JR. Surgical training: the impact of changes in curriculum and experience. J Surg Educ. 2011; 68: 44-51.

12. Shaw K, Cassel CK, Black C and Levinson W. Shared medical regulation in a time of increasing calls for accountability and transparency: comparison of recertification in the United States, Canada, and the United Kingdom. JAMA. 2009; 302: 2008-14.

13. Hout FA, Nienhuis ED, Robben PB, Frederiks BJ and Legemaate J. Supervision by the Dutch Healthcare Inspectorate. Eur J Health Law. 2010; 17: 347-60.

14. Issenberg SB, McGaghie WC, Hart IR, et al. Simulation technology for health care professional skills training and assessment. JAMA. 1999; 282: 861-6.

15. McDougall EM. Validation of surgical simulators. J Endourol. 2007; 21:244-7.

16. Scott DJ, Valentine RJ, Bergen PC, et al. Evaluating surgical competency with the American Board of Surgery In-Training Examination, skill testing, and intraoperative assessment. Surgery. 2000; 128: 613-22.

17. Clark RE, Feldon DF, van Merrienboer JJ, Yates KA and Early S. Cognitive Task Analysis. In: al SJMe, (ed.). Handbook of Research on Educational Communications and Technology. London: Taylor and Francis Group, 2008, p. 577-93. 


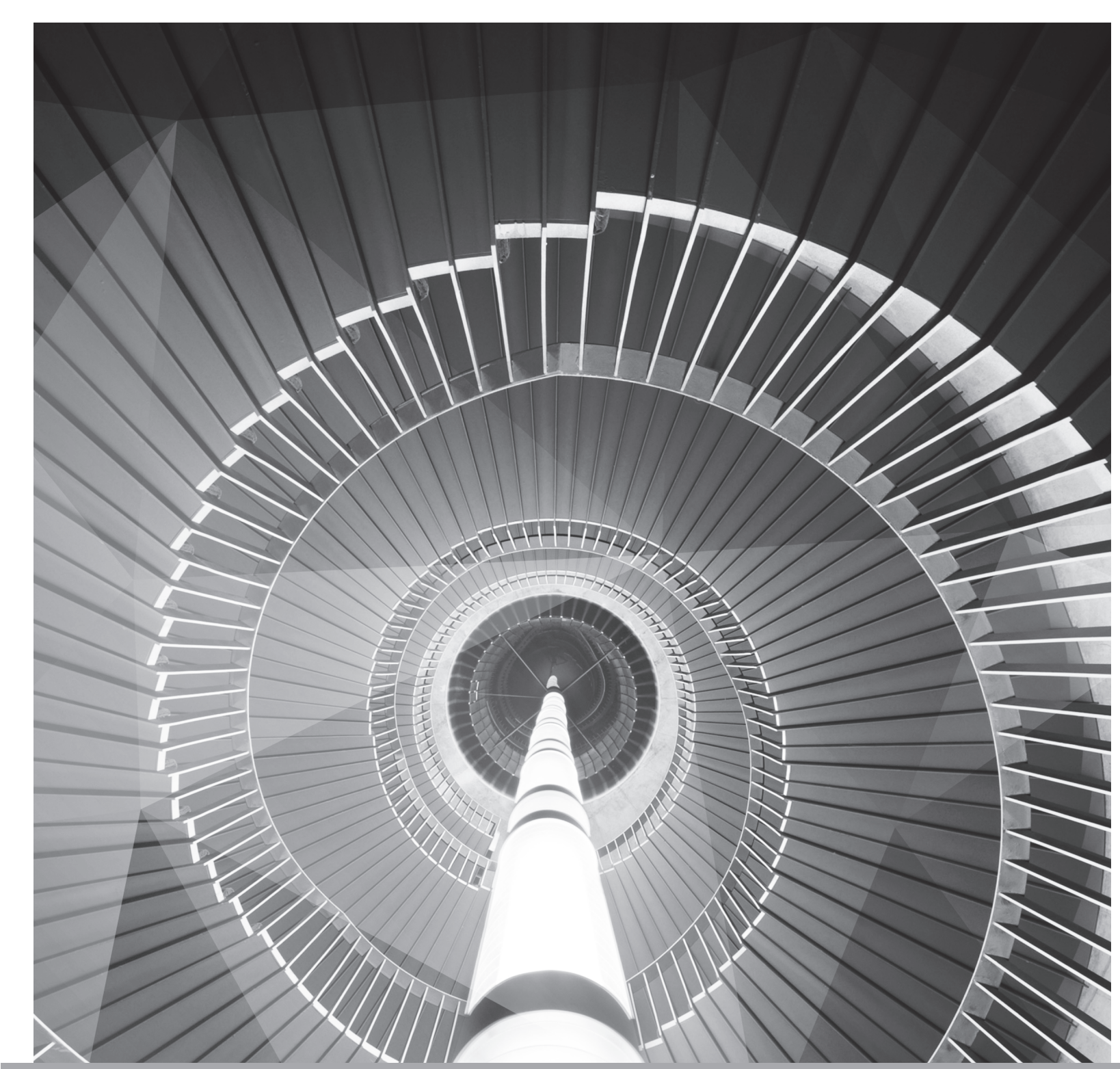

\section{Samenvatting}





\section{SAMENVATTING}

Minimaal invasieve procedures, zoals laparoscopie en endourologie, zijn algemene ingrepen binnen de urologie. Laparoscopie is een kijkoperatie van de buik, waarbij de instrumenten en een camera via kleine incisies in de patiënt worden gebracht. Bij endourologische procedures worden de instrumenten ingebracht via een natuurlijke opening, zoals in dit geval de urethra. In beide gevallen voert men de procedure uit door op een tv-monitor te kijken. In vergelijking met open operaties hebben alle vormen van minimaal invasieve chirurgie per definitie een langere leercurve. Dit komt onder andere door het instrumentarium, de verminderde tactiele feedback, een andere oog-hand coördinatie en de beperkte werkruimte waarmee de operateur moet leren omgaan. ${ }^{1-4}$

Het leren van chirurgische vaardigheden is van oudsher gebaseerd op het "meestergezel" model, waarbij de leerling eerst kijkt bij zijn meester, vervolgens de procedure mag doen onder supervisie, om uiteindelijk de procedure zelfstandig uit te voeren. ${ }^{5-8}$ Tegenwoordig is het niet meer zo eenvoudig als vroeger om alle chirurgische vaardigheden tot expert niveau in de praktijk te leren tijdens de opleiding. De arbeidstijdenwet van assistenten in opleiding en de ethische en juridische regelgeving beperken het aantal leermomenten aanzienlijk. ${ }^{9-11}$ Daarnaast wordt vanuit het algemene publiek en de wetgeving geëist dat er bepaalde bekwaamheidsnormen worden gesteld aan de snijdende specialist. Het wordt dan ook niet meer geaccepteerd dat een arts in opleiding zijn eerste stappen van een procedure direct op de patiënt leert. ${ }^{5,12,13}$

Onderwijskundigen zijn daarom op zoek naar alternatieve leermethoden om de conventionele manier van leren en toetsen van chirurgische vaardigheden te complementeren, zoals vaardigheidsonderwijs met behulp van simulatoren. De sleutel van vaardigheidsonderwijs met simulatoren is dat de leerling kan oefenen in een skillslab, fouten kan maken en hiervan kan leren zonder hierbij de gezondheid van een patiënt te schaden. $6,7,14$

Voordat een simulator in een bestaand onderwijsprogramma geïmplementeerd kan worden moet wel eerst onderzocht worden of de simulator effectief is. Met andere woorden, leert de simulator de vaardigheden wat men beoogt te leren? De effectiviteit en betrouwbaarheid van een simulator kan onderzocht worden middels een validatie proces. ${ }^{15}$

De overheid en het algemene publiek eisen tegenwoordig dat alle snijdende specialisten een bewijs van bekwaamheid kunnen overhandigen ten einde de patiëntveiligheid te waarborgen. Traditioneel gezien wordt bekwaamheid gerelateerd aan de aantallen die de specialist maakt; dus hoe meer hij opereert, hoe beter zijn kwaliteit van operaties wordt geacht. Echter, op volume gebaseerde normen om iemands bekwaamheid te toetsen zijn niet betrouwbaar. Een hoog volume wil niet automatisch betekenen dat al deze patiënten op de juiste manier werden geopereerd en de gewenste post-operatieve 
uitkomst hadden. ${ }^{8,16}$ De uitdaging in het ontwikkelen van een vaardigheidstoets is dan ook het stellen van de toetsnorm: wanneer is iemand gezakt en wanneer is iemand geslaagd?

Verder is het van belang om de plaats en de rol van de simulator in een bestaand curriculum te identificeren. Voor welk deel van welke procedure is de simulator geschikt als leermethode en op welke manier kan deze het beste geïntegreerd worden in het curriculum? Een "Training needs analyse" zou hiervoor uitgevoerd kunnen worden om de leerdoelen van de training vast te leggen.

Urologische vaardigheden kunnen worden beschouwd als complexe vaardigheden, waarbij handvaardigheid en kennis (cognitie) zijn geïntegreerd. Volgens de discipline "instructional design" worden complexe taken gedefinieerd als taken die voor de uitvoering een combinatie vereisen van geïntegreerde geautomatiseerde en nietgeautomatiseerde kennis en handvaardigheid. ${ }^{17}$ Het moeilijkste van een "training needs analyse" is het filteren van de procedurele stappen van een ingreep en de besluitvormingsprocessen die een uroloog maakt tijdens de ingreep. Daarmee kan bepaald worden voor welk deel van de procedure de simulator het meest geschikte trainingsmedium is. Strategieën afkomstig van de discipline "instructional design" kunnen bruikbaar zijn om een "training needs analyse" voor urologische ingrepen beter te structureren.

Minimaal invasieve urologie confronteert de uroloog niet alleen met een langere leercurve, maar ook met ergonomische uitdagingen vanwege de statische positie gedurende de gehele procedure. De wetenschap ergonomie analyseert deze uitdagingen en ontwerpt richtlijnen met als doel om een veilige werkomgeving te creëren voor de operateur en zijn team.

In dit proefschrift wordt de volgende onderzoeksvraag behandeld: Hoe kunnen training en toetsing met simulatoren ontwikkeld worden om het leren in de urologie te bevorderen en de kloof tussen onderzoek en integratie in de praktijk te verkleinen? (Hoofdstuk 1)

In hoofdstuk 2 worden de ontwikkeling en evaluatie van een gemodificeerd programma voor urologische laparoscopische vaardigheden, oftewel de "program for laparoscopic urological skills" (PLUS), beschreven. PLUS bestaat uit vijf basis taken, waarvan drie zijn overgenomen van de Fundamentals of Laparoscopic Surgery en twee taken zijn ontwikkeld onder continue evaluatie van expert urologen. Vijftig proefpersonen met verschillende ervaringsniveaus in laparoscopie voerden de vijf taken uit. Dit deden zij twee keer achter elkaar. Na het uitvoeren van de taken vulden de proefpersonen een vragenlijst in waarin ze konden aangeven hoe nuttig en waarheidsgetrouw ze de simulator vinden als trainingsmethode in de urologie.

De PLUS scoorde positief voor de Nederlandse setting volgens de mening van urologen, urologen in opleiding en beginners. De simulator kreeg hoge scores voor nut; 4.55 
op een schaal van 1 (=niet nuttig) tot 5 (=nuttig). Daarnaast werd er een sterke correlatie gevonden tussen ervaringsniveau en tijd. De verbetering in tijd voor experts versus beginners varieerde van $46 \%$ tot $74 \%$ voor alle vijf de taken. Er werden ook substantiële correlaties gemeten tussen ervaring en kwaliteit; en ervaring en aantal gevallen kralen. Een relatieve toename van $18 \%$ in kwaliteit werd gevonden voor experts versus beginners. Uit de resultaten blijkt dus dat men de PLUS een nuttige simulator vindt voor het urologische curriculum. Tevens kan de simulator onderscheid maken tussen personen met verschillende ervaringsniveaus in laparoscopie en heeft het voor met name diegene die nog aan het begin van hun leercurve zijn een substantieel leereffect.

In hoofdstuk 3 wordt de standaardisatie voor basis vaardigheden laparoscopie beschreven. Hiervoor hebben we eerst de betrouwbaarheid van de PLUS als toetsinstrument bepaald. Vervolgens werd de toetsnorm vastgesteld voor tweede jaar urologen in opleiding op basis van de "examinee-centered method". Deze onderwijskundige benadering gebruikt de lineaire relatie tussen de toetsscores en de ervaringsniveaus van verschillende referentiegroepen. De toetsscore op de grens van twee referentiegroepen wordt gebruikt als toetsnorm. In onze studie werden vijftig proefpersonen met verschillende ervaringsniveaus in laparoscopie getoetst op de PLUS met betrekking tot tijd- en kwaliteitscriteria. De generaliseerbaarheids coëfficiënt werd gebruikt om de betrouwbaarheid van het PLUS-examen te meten. Een generaliseerbaarheids coëfficiënt van 0.8 op een schaal van 1 werd beschouwd als voldoende voor een toetsinstrument. De toetsnorm werd gebaseerd op ervaring in termen van aantal uitgevoerde laparoscopische ingrepen: beginners, intermediates (1 - 100 laparoscopische ingrepen) en experts (>100 laparoscopische ingrepen). De vastgestelde toetsnorm werd vervolgens getest op 33 tweedejaar assistenten urologie. De resultaten tonen dat de PLUS een betrouwbaar toetsinstrument is. De toetsnorm werd vastgelegd op de grens beginners/intermediates en intermediates/experts. Dit resulteerde in een slagingspercentage van $63.64 \%$ respectievelijk 9.09\%. Samenvattend is de PLUS een betrouwbaar toetsinstrument met tijd- en kwaliteitscriteria voor basis vaardigheden laparoscopie met een slagingspercentage van $63 \%$ voor Nederlandse tweede jaar urologen in opleiding wanneer de toetsnorm wordt vastgelegd op de grens van beginners/intermediates. Hieruit concluderen we dat het PLUS examen met de toetsnorm vastgelegd op de grens van beginners/intermediates een verdedigbare norm is, welke als startpunt beschouwd kan worden van waaruit de assistenten zich verder kunnen ontwikkelen naar het volgende level van expertise in de laparoscopie.

Vervolgens wilden wij meten wat het niveau van basis laparoscopische vaardigheden is van urologen in opleiding op Europees niveau en of zij zouden slagen voor het European Basic Laparoscopic Urological Skills (E-BLUS) examen volgens de vastgelegde toetsnorm in Nederland. Hoofdstuk 4 beschrijft een studie waarin de toetsscores van 70 urologen in opleiding in hun laatste jaar uit verschillende landen in Europa werden 
geanalyseerd. De E-BLUS bestaat uit dezelfde vijf taken en dezelfde toetscriteria als de PLUS. Uit de resultaten blijkt dat slechts $4.2 \%$ van de kandidaten slaagt volgens de toetsnorm van het PLUS examen. Alhoewel de kwaliteitscriteria voldoende werd gehaald (slagingspercentage $=63 \%$ ), haalde het merendeel van de kandidaten de gevalideerde tijdscriteria niet. Eenenzestig procent van de examenkandidaten gaf aan dat zij geen gelegenheid hebben gehad om hun laparoscopische vaardigheden te trainen in de vier weken voorafgaand aan de toets. Deze studie toont aan dat het niveau van basisvaardigheden laparoscopie van laatste jaar assistenten in Europa erg laag is. Gebrek aan praktijkervaring werd niet gecompenseerd door training in het skillslab. De timing en setting van een dergelijk examen moet verder geëvalueerd worden in de toekomst. Regelmatig trainen van laparoscopische vaardigheden of een gedegen fellowship na de opleiding zou het niveau van laparoscopische vaardigheden moeten verhogen.

In hoofdstuk $\mathbf{5}$ wordt een validatie studie beschreven van een virtual reality simulator om transurethrale resecties van de prostaat (TURP) te trainen. Hierbij werd het bestaande urologische curriculum als uitgangspunt gebruikt. In deze studie waren de uitkomstmaten van het validatie proces niet enkel gebaseerd op de uitkomstmaten die door de simulator werden aangeboden, maar we hadden deze vooraf vastgelegd middels een "training needs analyse" (TNA). Een expert panel werd gevraagd om de TNA uit te voeren en zodoende de procedurele stappen en de valkuilen van de TURP ingreep te identificeren. De uitkomstmaten van de simulator werden vervolgens vergeleken met de resultaten van de TNA. Hieruit bleek dat 10 van de 22 procedurele stappen die resulteerden uit de TNA en 4 van de 11 valkuilen werden gedekt door de TURPsim. Voor het validatieproces werden 66 proefpersonen geïncludeerd; 22 beginners (studenten geneeskunde), 22 intermediates (urologen in opleiding en urologen die 1 - 50 complete TURP procedures hebben uitgevoerd) en 22 experts (urologen die $>50$ TURP procedures hebben uitgevoerd). Alle proefpersonen volgden een gestandaardiseerde training waarin de basis principes van de TURP en de werking van de simulator werden uitgelegd. Tevens mochten zij een paar vastgestelde deeltaak oefeningen doen om vervolgens twee complete TURP procedures uit te voeren op de simulator. Nadien vulden zij een vragenlijst in met betrekking tot nut en waarheidsgetrouwheid van de TURPsim. De resultaten tonen dat $93 \%$ van de proefpersonen de TURPsim een nuttig trainingsmodel vinden. Mediane scores met betrekking tot het nut van de simulator was hoog: 7.3 op een schaal van 1 (=niet nuttig) tot 10 (zeer nuttig). Intermediates en experts hadden een significant snellere resectie tijd en minder bloedverlies dan beginners. Ondanks de trainingssessie voorafgaand aan het uitvoeren van de twee TURP procedures, moesten beginners een prostaatkwab vaker re-reserceren en deden zij de resectie vaker in de verkeerde volgorde vergeleken met intermediates en experts. Een verklaring hiervoor zou kunnen zijn dat beginners te veel nieuwe informatie in een korte tijd tot zich moesten nemen en dat voor experts deze informatie voor een groot deel al geautomatiseerd 
is. De resultaten tonen ook dat experts vaker reserceren met suboptimaal zicht door bloedverlies in vergelijking met beginners. Reden hiervoor zou kunnen zijn dat experts uit ervaring weten waar ze veilig kunnen reserceren. Uit dit onderzoek blijkt dus dat de simulator niet alle procedurele stappen en valkuilen dekt. Tevens kan de simulator niet differentiëren tussen geautomatiseerde en niet-geautomatiseerde vaardigheden. Desondanks heeft de TURPsim een positieve waardering gekregen wat betreft waarheidsgetrouwheid en nut voor het urologische curriculum. Daarnaast kan de simulator ook discrimineren tussen personen met verschillende ervaringsniveaus. Dit suggereert dat de TURPsim een geschikte simulator is voor het huidige urologische curriculum om de basis vaardigheden en de procedurele stappen van de TURP te leren.

TNA is een belangrijke onderdeel in het ontwerpproces en de evaluatie van training en toetsing van urologische vaardigheden. In hoofdstuk 6 wordt beschreven hoe een TNA voor het trainen van de nefrostomie procedure kan worden gestructureerd door middel van het integreren van het 4C/ID model en cognitieve taak analyse. Het doel van de nefrostomie procedure is om direct urine te draineren in geval van post-renale obstructie door een toegang te prikken via de huid in de nier. Het 4C/ID model beoogt planmatig automatische en niet-automatische stappen en de valkuilen van een procedure of ingreep te identificeren. Cognitieve training analyse omvat een variëteit aan observatie en interview methoden om te ontrafelen hoe experts complexe taken uitvoeren, waarbij de kennis, de besluitvormingsprocessen en de intenties tijdens een procedure worden uitgefilterd en vastgelegd. In dit onderzoek werden vier urologen benaderd om een vragenlijst in te vullen ten aanzien van de procedurele stappen. Daarna werden acht urologen uitgenodigd om deel te nemen aan een semi-gestructureerde interview. Het interview werd geleid door een onderzoeker, een onderwijskundige en een expert uroloog op het gebied van het aanprikken van een nefrostomie. Tijdens het semi-gestructureerde interview werden alle stappen en sub-stappen van de nefrostomie procedure gedefinieerd en elke (sub)-stap werd geclassificeerd als automatisch of niet-automatisch. Daarnaast werd gevraagd om scenario's te benoemen met verschillende moeilijkheidsgraad.

Het uiteindelijke resultaat van dit onderzoek was een blauwdruk bestaande uit volledige taken met oplopende moeilijkheidsgraad en uit deeltaken voor vaardigheden die een hoog niveau van automatisme vereisen. In de toekomst zal TNA voor verschillende procedures binnen de urologie uitgevoerd moeten worden ter bevordering van de ontwikkeling van training programma's en om de plaats van de trainingprogramma's in het huidige curriculum vast te leggen.

In hoofdstuk 7 wordt de focus gelegd op de ergonomie bij minimaal invasieve urologie. Een online vragenlijst en een vragenlijst op papier werd verstrekt onder urologen, afkomstig uit verschillende landen, die endourologie en/of laparoscopie uitvoeren in de dagelijkse praktijk. Onder de 285 respondenten, ervoeren 245 (86.0\%) lichamelijke 
klachten in de afgelopen 12 maanden en $62.1 \%$ daarvan gaf aan dat deze gerelateerd zijn aan hun werk. Een toename van de leeftijd was gerelateerd aan vermindering van klachten. De lichaamsdelen die het meest werden genoemd onder de chronische klachten waren: nek, rug en schouders. Bijna 50\% van de urologen hadden chronische klachten, waarvoor endourologie en laparoscopie significante risico factoren zijn. Een derde van de respondenten gaf aan dat ze weinig kennis hadden over ergonomie in de operatiekamer. Onze aanbeveling luidt dan ook om ergonomie te integreren in training programma's, bij voorkeur in het begin van de urologie opleiding, ten einde inzicht en bewustzijn te vergroten om uiteindelijk ergonomische klachten in de toekomst te voorkomen.

In hoofdstuk 8 worden de conclusies van de onderzoeken beschreven, gevolgd door een algemene discussie over de ontwikkeling van training en toetsing met behulp van simulatoren. Tevens worden de implicaties van ergonomische klachten onder urologen nader besproken. Tot slot komen enkele toekomstperspectieven en aanbevelingen voor verder onderzoek aan bod. 


\section{REFERENTIES}

1. Kumar U and Gill IS. Learning curve in human laparoscopic surgery. Curr Urol Rep. 2006; 7: 120-4.

2. Capelouto CC and Kavoussi LR. Complications of laparoscopic surgery. Urology. 1993; 42: 2-12.

3. Kerbl K and Clayman RV. Advances in laparoscopic renal and ureteral surgery. Eur Urol. 1994; 25: $1-6$.

4. Gallagher AG, McClure N, McGuigan J, Ritchie K and Sheehy NP. An ergonomic analysis of the fulcrum effect in the acquisition of endoscopic skills. Endoscopy. 1998; 30: 617-20.

5. Kohn LT, Corrigan JM and Donaldson MS. To err is human: Building a safer health system. 7 ed. Washington DC: National Academy Press, 2008.

6. Dornan T, Mann K, Scherpbier AJ and Spencer J. Medical Education: Theory and Practice. London: Churchill Livingstone Elsevier, 2011.

7. Rodriguez-Paz JM, Kennedy M, Salas E, et al. Beyond "see one, do one, teach one": toward a different training paradigm. Qual Saf Health Care. 2009; 18: 63-8.

8. Reznick RK and MacRae H. Teaching surgical skills_changes in the wind. N Engl J Med. 2006; 355: 2664-9.

9. Kelly BD, Curtin PD and Corcoran M. The effects of the European Working Time Directive on surgical training: the basic surgical trainee's perspective. Ir J Med Sci. 2011; 180: 435-7.

10. Scott DJ. Patient safety, competency, and the future of surgical simulation. Simul Healthc. 2006; 1 : 164-70.

11. Parsons BA, Blencowe NS, Hollowood AD and Grant JR. Surgical training: the impact of changes in curriculum and experience. J Surg Educ. 2011; 68: 44-51.

12. Shaw K, Cassel CK, Black C and Levinson W. Shared medical regulation in a time of increasing calls for accountability and transparency: comparison of recertification in the United States, Canada, and the United Kingdom. JAMA. 2009; 302: 2008-14.

13. Hout FA, Nienhuis ED, Robben PB, Frederiks BJ and Legemaate J. Supervision by the Dutch Healthcare Inspectorate. Eur J Health Law. 2010; 17: 347-60.

14. Issenberg SB, McGaghie WC, Hart IR, et al. Simulation technology for health care professional skills training and assessment. JAMA. 1999; 282: 861-6.

15. McDougall EM. Validation of surgical simulators. J Endourol. 2007; 21: 244-7.

16. Scott DJ, Valentine RJ, Bergen PC, et al. Evaluating surgical competency with the American Board of Surgery In-Training Examination, skill testing, and intraoperative assessment. Surgery. 2000; 128: 613-22.

17. Clark RE, Feldon DF, van Merrienboer JJ, Yates KA and Early S. Cognitive Task Analysis. In: al SJMe, (ed.). Handbook of Research on Educational Communications and Technology. London: Taylor and Francis Group, 2008, p. 577-93. 



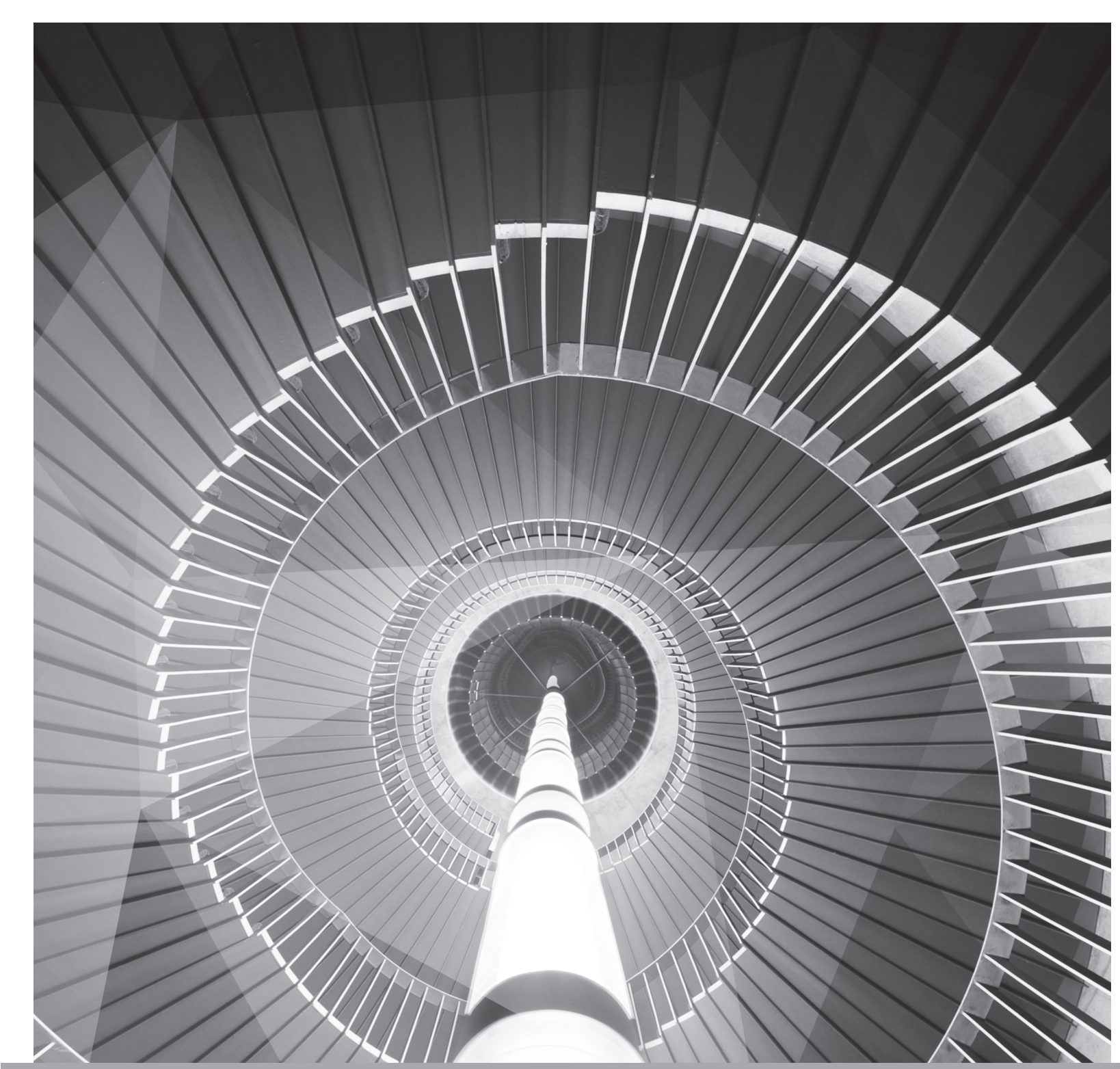

Curriculum Vitae 



\section{CURRICULUM VITAE}

Irene Tjiam was born in Goes on November $16^{\text {th }} 1979$. After secondary school education at st-Willibrord college (VWO) in Goes, she studied Health Sciences at Maastricht University for five years. During this period she followed a trimester of a master program in primary healthcare at Manchester University (UK). She wrote her master thesis at Zorgverzekeraars Nederland on the topic; Mobility of the Dutch with private health insurances within the European Union. In 2003 she received her masters degree in Health Sciences, specialization: health policy and

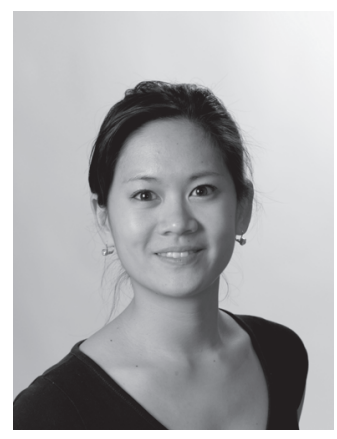
administration.

In 2003 she started medical school at Maastricht University. During this period she did several rotations in different hospitals in the Netherlands and one rotation in Mtwara, Tanzania. After graduation in 2009 she started working as a resident in urology not in training (ANIOS) and as a PhD candidate on the project "Training in Urology", resulting in this thesis, at the Catharina Hospital in Eindhoven, the Netherlands. During this period she attended several conferences and courses in the Netherlands and abroad. She organised the teach-the-teacher courses for the hands-on-training tutors of the EUREP in Prague $(\mathrm{Cz})$. Furthermore she implemented the PLUS examination in the Netherlands and the E-BLUS in Europe. Also she organised the dry-lab training of the mandatory laparoscopic course for Dutch residents in urology in Elancourt (Fr) and attended the hands-on training courses in the Catharina Hospital.

On January $1^{\text {st }} 2012$ until $31^{\text {st }}$ of December 2013 she fulfilled her surgical part of formal postgraduate training in urology at the department of general surgery at the Maxima Medical Centre in Veldhoven, the Netherlands (programme directors: Dr. Prakken, Dr. RM Roumen and Dr. MR Scheltinga). Currently she works as a resident in urology at the RadboudUMC Nijmegen, the Netherlands (programme director: Prof. JA Witjes). 



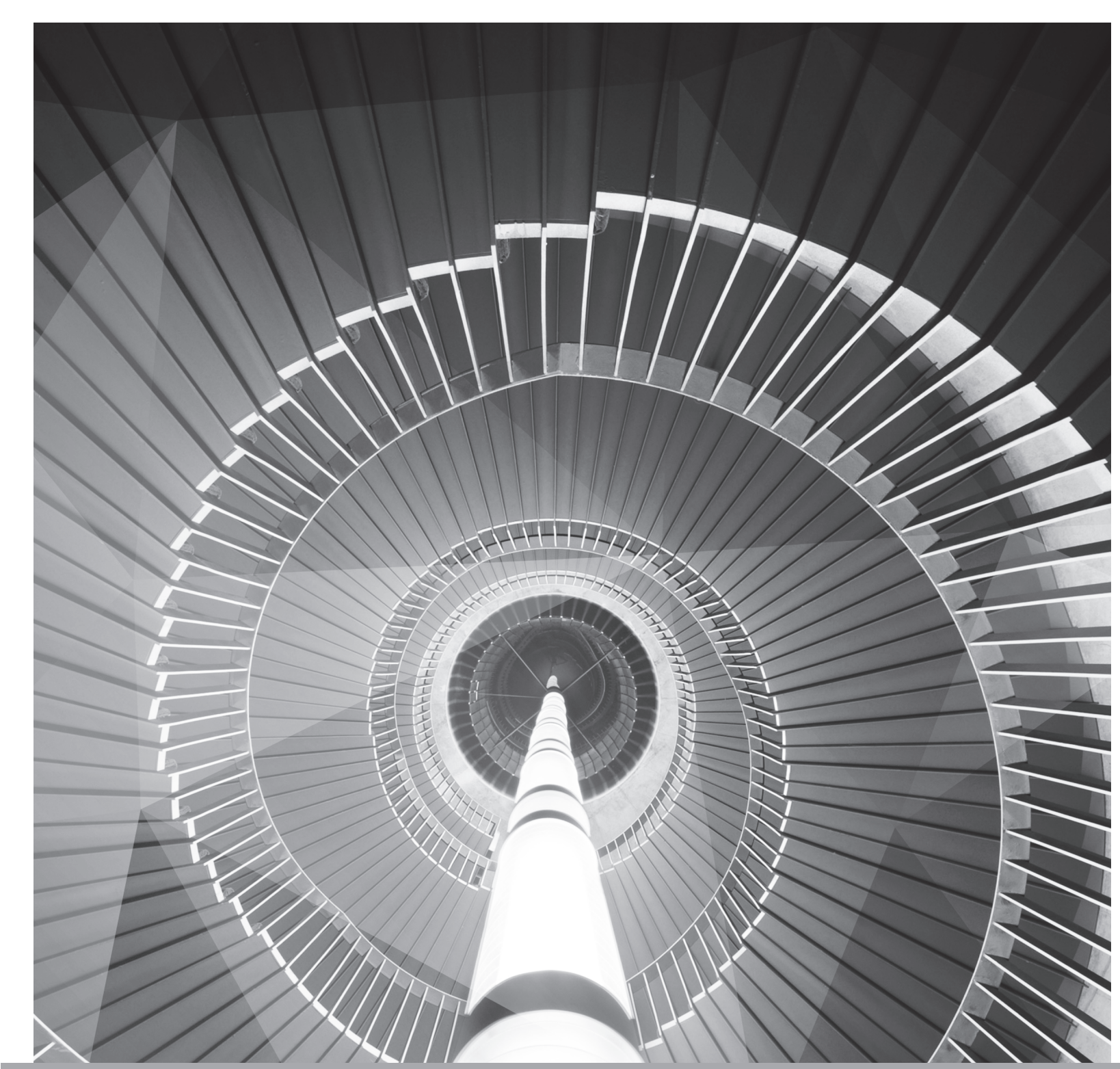

\section{List of Publications}





\section{LIST OF PUBLICATIONS}

Tjiam IM, Berkers CH, Schout BM, Brinkman WM, Witjes JA, Scherpbier AJ, Hendrikx AJ, Koldewijn EL. Evaluation of the educational value of a virtual reality TURP simulator according to a curriculum based approach. Simulation in Healthcare 2014; accepted

Tjiam IM, Goossens RH, Schout BM, Koldewijn EL, Hendrix AJ, Muijtjens AM, Scherpbier AJ, Witjes JA. Ergonomics in endourology and laparoscopy: An overview of musculoskeletal problems in urology. J Endourol. 2014: 28(5): 605-11.

Brinkman WM, Tjiam IM, Schout, BM, Muijtjens AM, van Cleynenbreugel B, Koldewijn EL, Witjes JA. Results of the European Basic Laparoscopic Urological Skills Examination. Eur. Urol. 2014:65(2):490-6.

Brinkman WM, Tjiam IM, Buzink SN. Assessment of basic laparoscopic skills on virtual reality simulator or box trainer. Surg Endosc. 2013:27(10):3584-90.

Tjiam IM, Schout BM, Hendrikx AJ, Muijtjens AM, Scherpbier AJ, Witjes JA, van der Vleuten CP. Program for laparoscopic urological skills assessment: setting certification standards for residents. Minim Invasive Ther Allied Technol. 2013:22(1):26-32.

van Merriënboer JJ en Tjiam IM. H12 Development and Teaching of Complex Skills in Invasive Procedures. In: Catheter-Based Cardiovascular intervention; Knowledge based approach. Springer Heidelberg 2013.

Tjiam IM, Persoon MC, Hendrikx AJ, Muijtjens AM, Witjes JA, Scherpbier AJ. Program for laparoscopic urological skills: a newly developed and validated educational program. Urology 2012:79(4):815-20.

Tjiam IM, Schout BM, Hendrikx AJ, Scherpbier AJ, Witjes JA, van Merrienboer JJ. Designing simulator-based training: an approach integrating cognitive task analysis and fourcomponent instructional design. Med Teach. 2012:34(10):e698-707.

Persoon MC, Schout BM, Martens EJ, Tjiam IM, Tielbeek AV, Scherpbier AJ, Witjes JA, Hendrikx AJ. A simulator for teaching transrectal ultrasound procedures: how useful and realistic is it? Simul Healthc. 2010:5(5): 311-14.

van Basten JP, Beerlage H, Tjiam IM. Aanbevelingen Laparoscopie in de Urologie. Verhagen Grafisch Media Veldhoven 2010. 
Tjiam IM, Persoon MC, Hendrikx AJ. H7 Opleiding en Training. In: Aanbevelingen Laparoscopie in de urologie. Verhagen Grafisch Media Veldhoven 2010.

Hendrikx AJ en Tjiam IM. Eerste diploma's "basis laparoscopische urologische skills" uitgereikt aan assistenten urologie onder toeziend oog van de inspectie. Urograaf 2009. 


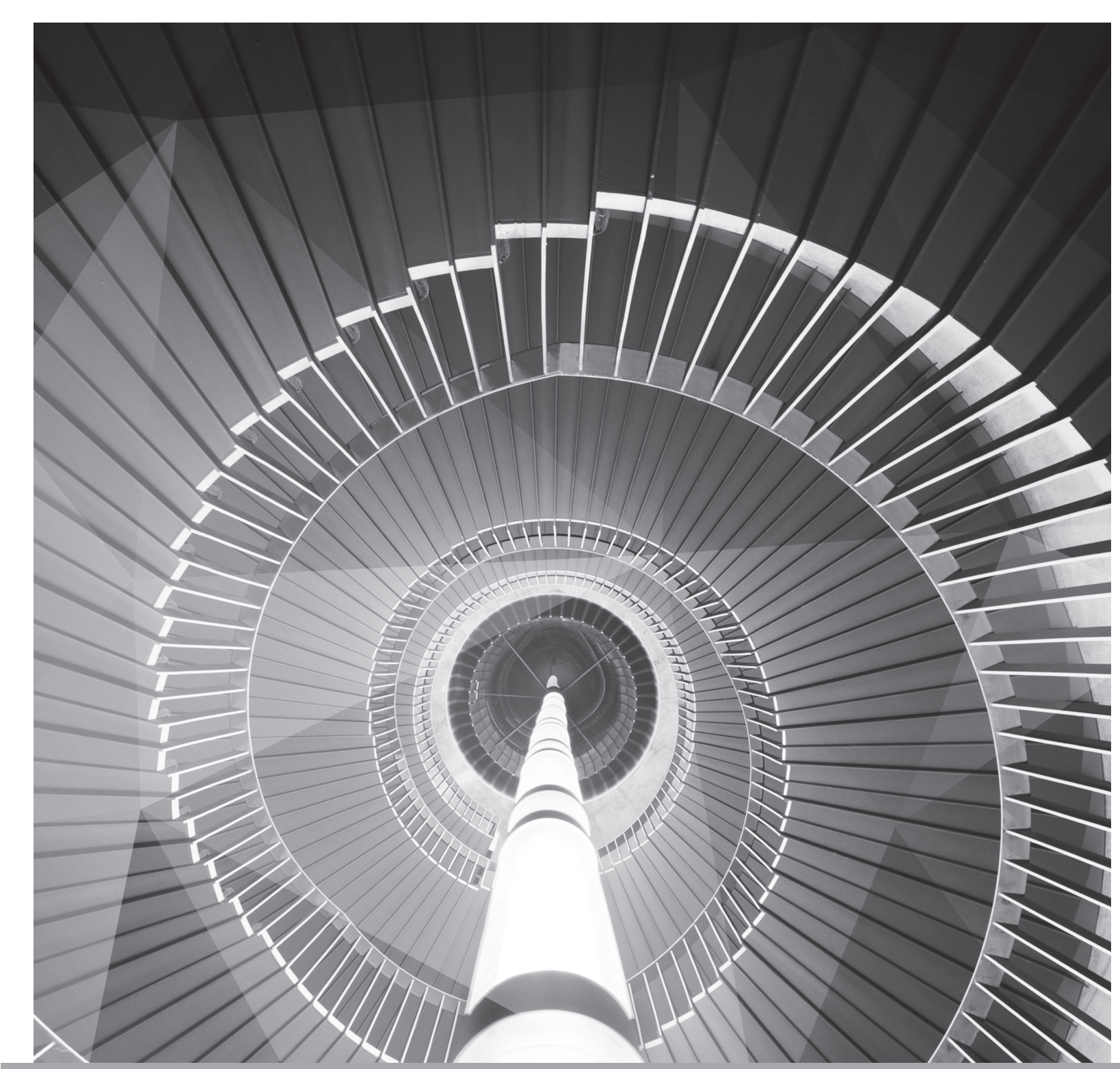

\section{Dankwoord}





\section{DANKWOORD}

In 2009 ben ik begonnen aan dit promotie traject. Dat mijn proefschrift ooit af zou komen had ik nooit durven dromen! Ik heb veel geleerd, dat is zeker. Het promotietraject heeft mij enorm veel kansen geboden om mij te ontplooien als wetenschapper, organisator, initiator en als arts. Dankzij de hulp van een heleboel mensen is het mij gelukt om dit project tot een goed einde te brengen. Hierbij neem ik de kans om een aantal mensen in het bijzonder te bedanken.

"Geduld betekent niet dat je de dingen passief over je heen laat komen. Het betekent dat je ver genoeg vooruit kunt kijken om vertrouwen te hebben in het eindresultaat van een proces" (Elif Shafak, 2010)

Beste prof. Scherpbier, Albert. Wat heb ik veel bewondering voor jouw geduld, vertrouwen en niet aflatende interesse in je promovendi. Ondanks dat je het ontzettend druk hebt zowel in je werk als privé, lukt het je altijd om tijd vrij te maken voor een mailtje of een belletje. Of het nou een doordeweekse dag of weekend is, het maakt niet uit. Daarnaast leken de mogelijkheden om mij te ontplooien binnen het project bijna grenzeloos. Heel veel dank voor je wetenschappelijke adviezen op het gebied van medisch onderwijs en vooral veel dank voor je vertrouwen in mij.

Beste prof. Witjes, Fred. Promotor en opleider; heel veel waardering heb ik voor je kritische blik, je eerlijkheid en je openheid. What you see is what you get! Als promotor was je onmisbaar in het project. Jij was degene die kritisch de keerzijde kon belichten van elk nieuw idee, maar jij was ook degenen die de opening bood om het project tot internationaal niveau te tillen. Inmiddels ben je mijn opleider in de urologie en ik hoop de komende jaren nog heel veel van je te leren!

Beste dr. Hendrikx, Ad. Wat ben ik blij om zo'n bijzonder persoon te mogen kennen! Hoe jij nieuwe initiatieven ontplooid is ongelofelijk knap en ik hoop dat ik daar ook wat van heb meegekregen voor de toekomst. Heel veel dank dat je mij hebt geïntroduceerd in de wereld van de urologie en het urologisch onderwijs op nationaal en internationaal niveau. Je hebt de deuren voor mij geopend en ik hoefde alleen maar binnen te stappen. Ik ben de vierde in de rij van promovendi en hopelijk volgen er nog veel meer.

Beste dr. Schout, Barbara. Bubbels! Gelukkig is dat het eerste waar ik aan denk. Maar je bent ook een strenge, correcte en gedisciplineerde co-promotor. Wat heb ik ontzettend veel aan je wetenschappelijke adviezen gehad. Ik vond het erg nuttig om met je te brainstormen en te sparren over mijn studies. Bedankt voor alle hulp! 
Beste dr. Koldewijn, Evert. Inmiddels heb je een grote rol binnen het project. Echter daarvoor was je ook zeer betrokken en altijd enthousiast om mee te helpen en mee te denken. Ik kan me nog een ritje naar een NVU voorjaarsvergadering herinneren om een onmogelijk grote simulator af te leveren. Dank voor al je hulp en enthousiasme!

Beste dr. Brinkman, Willem. Doctor B, daar staan we dan in 2014 zonder "s". We hebben een aantal artikelen samen geschreven, en ik hoop dat we dat in de toekomst nog kunnen blijven doen. Bedankt dat je mijn paranimf wil zijn. Heel veel succes met je opleiding collega!

Beste Heleen, wat ben jij een gezellig mens! En wat fijn dat je in mijn huisje bent komen wonen. Ik heb er alle vertrouwen in dat jouw promotie helemaal goed gaat komen en ik kijk nu al uit naar je feestje! Bedankt dat je mijn paranimf wilt zijn!

Beste Marjolein, mijn onderzoekscollega en theemaatje in mijn eerste jaar. Je hebt mij wegwijs gemaakt in dit onderzoek. Maar wat vooral niet vergeten mag worden: jij hebt de aller-allereerste stap gezet in het BLUS-project.

Carsten Mellema, tot op de dag van vandaag weet ik nog precies waar ik was toen jij me belde over dit project en dat ik maar even telefonisch contact moest opnemen met Barbara Schout omdat de officiële sollicitatie al gesloten was. Jij bent eigenlijk de initiator van dit eindresultaat. Heel veel dank voor de ogenschijnlijk kleine tip waardoor ik hier nu sta.

Urologen en arts assistenten van het CzE: Henry, Rik, Pascal, Rob, Kim, Hans, Ward Nele, Eric Vrijhof, Evert Koldewijn, Michel de Wildt, Wout Scheepens. ledereen bedankt dat jullie steeds opnieuw en vol enthousiasme wilden deelnemen als proefpersoon. Daarnaast heel veel dank voor alle gezelligheid, geduld en begeleiding in de kliniek. Ik heb een hele mooie tijd gehad, veel gelachen en bovenal heel veel geleerd.

Zonder proefpersonen is een promovendus nergens. Ik wil hierbij alle studenten, AIOS en ANIOS urologie en urologen bedanken voor hun deelname aan mijn studies.

Ook wil ik de urologen van de EUREP bedanken dat ze wilden deelnemen aan het EBLUS onderzoek.

Graag wil ik de heren van de SWEN hartelijk bedanken voor hun tijd en moeite om aan mijn onderzoeken deel te nemen. Ik heb jullie leren kennen als hardwerkende expert urologen, maar ook als heren die van gezelligheid en lekker eten houden! Daarnaast, 
omdat dit waarschijnlijk toch nooit vergeten gaat worden: Ben, wanneer gaan we nog eens naar Parijs?

Hierbij wil ik een groot aantal mensen bedanken van het CzE: alle mensen van het UBC dank voor jullie medewerking aan het BLUS-examen. Jullie doen het nog steeds top! Alle secretaresses van de urologie en verpleegkundigen van 8-Oost bedankt voor jullie hulp en samenwerking. Manon, wanneer gaan we nog eens thee drinken?

Angelique Dierick en Gert de Vroom bedankt voor de goede samenwerking in het skillslab!

Mereke Gorsira, recent heb ik vernomen dat het leven je is ontnomen. Graag wil ik mijn dank aan jou op papier zetten. Als english editor heb je mijn eerste artikelen tot een publicatiewaardig niveau vertaald, heel veel dank hiervoor. Arno Muijtjens, bedankt voor jouw statistische kennis en verrassende inzichten!

Chirurgen en collega's assistenten uit het Maxima Medisch Centrum, bedankt voor de gezellige tijd en alle leerzame momenten in de kliniek. Jullie hebben me letterlijk de eerste stapjes op de ok geleerd!

Urologen en collega assistenten uit het Radboud umc, ik heb er zin in!

Beste mannen van Kinran, bedankt voor alle mooie momenten al dan niet onder het genot van een biertje in Maastricht en niet te vergeten in Frankrijk (ik krijg nog steeds een glimlach op mijn gezicht als ik daaraan terugdenk). Ook veel dank voor de hulp tijdens alle verhuizingen. Sensei Peter: "Oss!".

Lieve Carlijn, Renee, Hanne, Sera, Frederique, Charlotte en Dirk. Waar zou ik zijn zonder jullie? Jullie hebben me echt gesteund in voor en tegenspoed. De afgelopen jaren waren als een rollercoaster en ik heb echt veel aan jullie gehad. Dat er nog maar veel drankjes/ etentjes/weekendjes/fesitivals mogen komen in de toekomst!

Lieve mama en papa, Angela en Maarten. Jullie zijn er altijd voor mij en dat is zo waardevol! Mama en papa, bedankt voor al jullie steun, regel dingen, praktische zaken en verhuizingen. Ook bedankt voor het lekkere eten en mijn altijd overvolle koelkast en diepvries! An en Maarten, ik voel me altijd zo welkom bij jullie in Rotterdam. En ik ben heel blij dat je binnenkort mijn schoonbroer bent Maarten. 
Lieve Angela, bedankt dat je mijn extreme vakantie buddy bent geweest in de afgelopen jaren. We hebben heel wat (letterlijke en figuurlijke) dieptepunten besproken en hoogtepunten overwonnen in de afgelopen jaren. Twee jaar geleden hebben we Mnt. Kilimanjaro overwonnen. Vorige jaar de Yellow Mountains en dit jaar gaan we samen naar Mnt. Everest Base Camp. Ik ben heel trots op mijn kleine zus, hoe jij dat de afgelopen jaren hebt gedaan. Met jouw werk ethos en strakke relax modus ga je het helemaal maken in de oogheelkunde!

Aan alle anderen die mijn leven de afgelopen jaren een rijker mens hebben gemaakt, heel veel dank! 


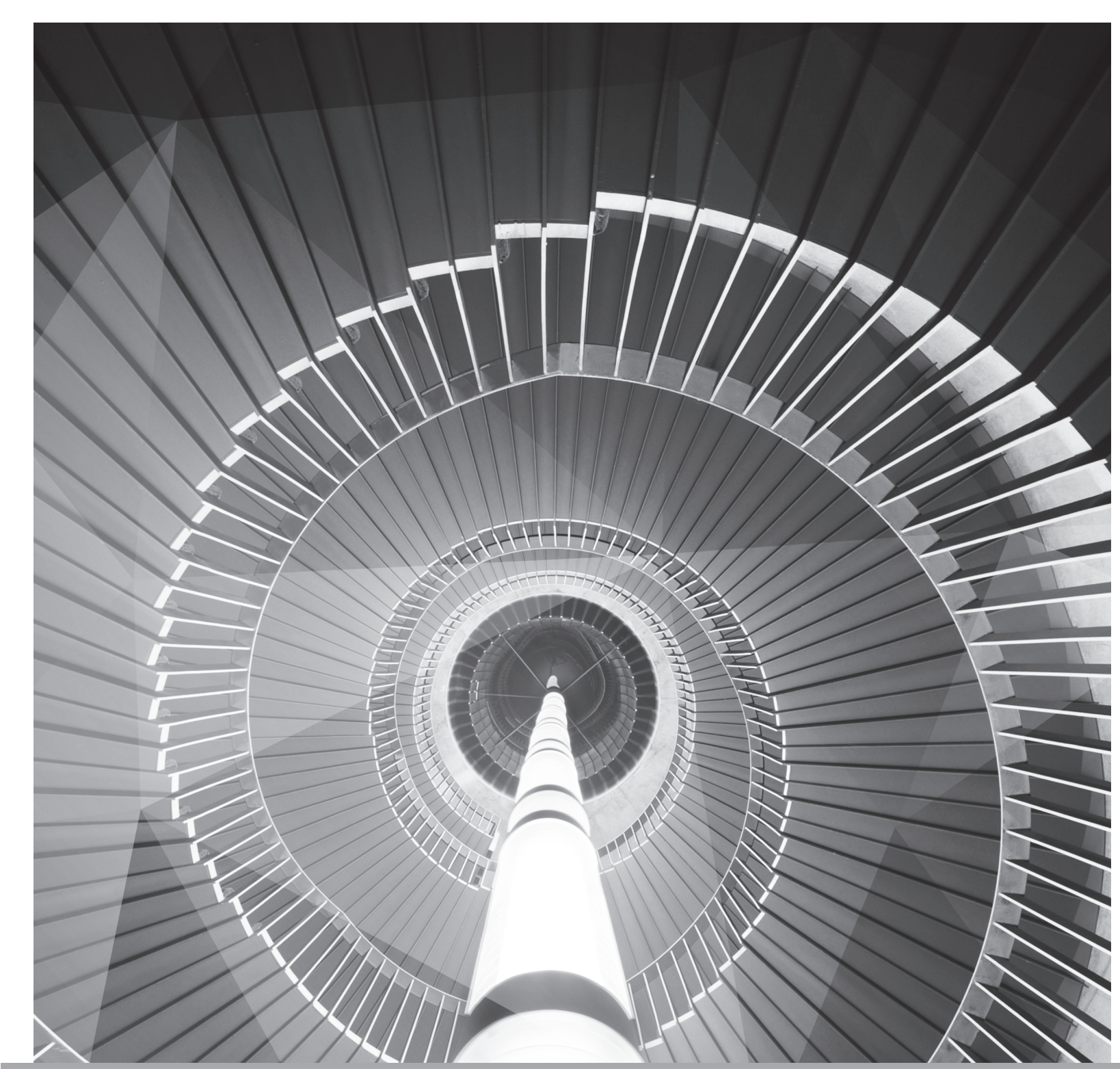

SHE Dissertation Series 

The SHE Dissertation Series publishes dissertations of PhD candidates from the School of Health Professions Education (SHE) who defended their PhD theses at Maastricht University. The most recent ones are listed below. For more information go to: www. maastrichtuniversity.nl/she.

Van Loon, M.H. (08-05-2014) Fostering monitoring and regulation of learning

Frambach, J.M. (26-03-2014) The cultural complexity of problem-based learning across the world

Hommes, J.E. (26-02-2014) How relations, time \& size matter in medical education

Van der Zwet, J. (30-01-2014) Identity, Interaction and Power. Explaining the affordances of doctor-student interaction during clerkships

Watling, C.J. (22-01-2014) Cognition, Culture, and Credibility. Deconstructing Feedback in Medical Education

Winston, K. (12-12-2013) Remediation Theory and Practice: Transforming At-Risk Medical Students

Kamp, R.J.A. (28-11-2013) Peer Feedback to Enhance Learning in Problem-Based Tutorial Groups

Junod Perron, N. (24-10-2013) Towards a learner-centered approach to postgraduate communications skills teaching

Pratidina Susilo, A. (24-10-2013) Learning to be the Patient Advocate

The Development of a Communication Skills Course to Enhance Nurses' Contribution to the Informed Consent Process

Alves de Lima, A. (23-10-2013) Assessment of clinical competence: Reliability, Validity, Feasibility and Educational Impact of the mini-CEX

Sibbald, M. (09-10-2013) Is that your final answer? How doctors should check decisions

Ladhani, Z. (05-07-2013) Competency based education and professional competencies: a study of institutional structures, perspectives and practices in Pakistan 
Jippes, M. (01-02-2013) Culture matters in medical schools: How values shape a successful curriculum change

Duvivier, R. J. (12-12-2012) Teaching and Learning Clinical Skills. Mastering the Art of Medicine

De Feijter, J.M. (09-11-2012) Learning from error to improve patient safety

Prescott, L. (09-11-2012) Ensuring the Competence of Dental Practitioners through the Development of a Workplace-Based System of Assessment

Cilliers, F.J. (05-09-2012) The Pre-assessment Learning Effects of Consequential Assessment: Modelling how the Examination Game is Played

Spanjers, I. A.E. (05-07-2012) Segmentation of Animations: Explaining the Effects on the Learning Process and Learning Outcomes

Al-Kadri, H.M.F. (28-06-2012) Does Assessment Drive Students' Learning?

Leppink, J. (20-06-2012) Propositional manipulation for conceptual understanding of statistics

Van Zundert, M.J. (04-05-2012) Conditions of Peer Assessment for Complex Learning

Claramita, M. (30-03-2012) Doctor-patient communication in a culturally hierarchical context of Southeast Asia: A partnership approach

Kleijnen, J.C.B.M. (21-03-2012) Internal quality management and organizational values in higher education

Persoon, M.C. (19-01-2012) Learning in Urology; The influence of simulators and human factors

Pawlikowska, T.R.B. (21-12-2011) Patient Enablement; A Living Dialogue

Sok Ying Liaw, (14-12-2011) Rescuing A Patient In Deteriorating Situations (RAPIDS): A programmatic approach in developing and evaluating a simulation-based educational program 
Singaram, V.S. (7-12-2011) Exploring the Impact of Diversity Factors on Problem-Based Collaborative Learning

Balslev, T. (24-11-2011) Learning to diagnose using patient video cases in paediatrics: Perceptive and cognitive processes

Widyandana, D. (19-10-2011) Integrating Pre-clinical skills training in skills laboratory and primary health care centers to prepare medical students for their clerkships

Durning, S.J. (09-09-2011) Exploring the Influence of Contextual Factors of the Clinical Encounter on Clinical Reasoning Success (Unraveling context specificity)

Govaerts, M.J.B. (08-09-2011) Climbing the Pyramid;Towards Understanding Performance Assessment

Stalmeijer, R. E. (07-07-2011) Evaluating Clinical Teaching through Cognitive Apprenticeship

Malling, B.V.G. (01-07-2011) Managing word-based postgraduate medical education in clinical departments

Veldhuijzen, J.W. (17-06-2011) Challenging the patient-centred paradigm: designing feasible guidelines for doctor patient communication

Van Blankenstein, F. (18-05-2011) Elaboration during problem-based, small group discussion: A new approach to study collaborative learning

Van Mook, W. (13-05-2011) Teaching and assessment of professional behavior: Rhetoric and reality

De Leng, B. (8-12-2009). Wired for learning. How computers can support interaction in small group learning in higher education

Maiorova, T. (29-05-2009). The role of gender in medical specialty choice and general practice preferences

Bokken, L. (04-03-2009). Innovative use of simulated patients for educational purposes 
Wagenaar, A. (18-09-2008). Learning in internships. What and how students learn from experience

Driessen, E. (25-06-2008). Educating the self-critical doctor. Using portfolio to stimulate and assess medical students' reflection

Derkx, H. (18-06-2008). For your ears only. Quality of telephone triage at out-of-hours centres in the Netherlands

Niessen, Th. (30-11-2007). Emerging epistemologies: making sense of teaching practice

Budé, L. (05-10-2007). On the improvement of students' conceptual understanding in statistics education

Niemantsverdriet, S. (26-07-2007). Learning from international internships: A reconstruction in the medical domain

Marambe, K. (20-06-2007). Patterns of student learning in medical education-A Sri Lankan study in traditional curriculum

Pleijers, A. (19-01-2007). Tutorial group discussion in problem-based learning

Sargeant, J. (21-09-2006). Multi-source feedback for physician learning and change

Dornan, T. (12-06-2006). Experience-based learning

Wass, V. (12-05-2006). The assessment of clinical competence in high stakes examinations

Prince, K. (21-04-2006). Problem-based learning as a preparation for professional practice 



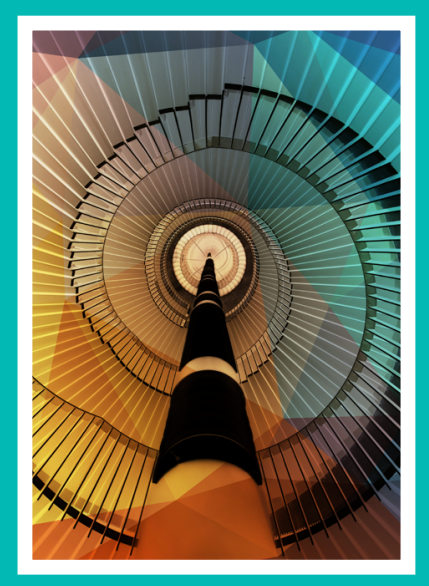

